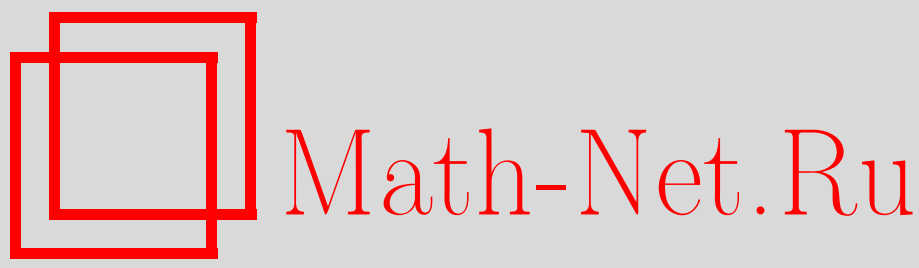

М. В. Карасёв, А. В. Перескоков, Асимптотические решения уравнений Хартри, сосредоточенные вблизи маломерных подмногообразий. II. Локализация в плоских дисках, Изв. РАН. Сер. матем., 2001, том 65, выпуск 6, 57-98

DOI: https://doi.org/10.4213/im365

Использование Общероссийского математического портала Math-Net.Ru подразумевает, что вы прочитали и согласны с пользовательским соглашением

http://www.mathnet.ru/rus/agreement

Параметры загрузки:

IP: 3.93 .64 .190

26 апреля 2023 г., 06:43:57 
УДК 517.946

М.В. Карасев, А. В. Перескоков

\title{
Асимптотические решения уравнений Хартри, сосредоточенные вблизи маломерных подмногообразий. II. Локализация в плоских дисках
}

\begin{abstract}
Рассматривается задача нахождения собственных значений для трехмерного уравнения Хартри во внешнем поле. Построены асимптотические (квазиклассические) решения, сосредоточенные вблизи плоских двумерных дисков. Убывание этих решений по нормали к диску определяется поляроном Боголюбова, а вблизи края диска задается эйри-аналогом полярона. Для нахождения соответствующей серии собственых значений получен аналог правила квантования Бора-Зоммерфельда, из которого выведено более простое алгебраическое уравнение, определяющее главные члены асимптотики собственных значений.

Библиограффия: 10 наименований.
\end{abstract}

\section{$\S 1$. Введение}

Данная статья является второй, заключительной, частью работы [1]. Рассматривается следующая задача на собственные значения для нелинейного уравнения типа Хартри:

$$
\begin{gathered}
\varepsilon^{2} \Delta \psi(x)+\left(-a|x|^{\alpha}+\int_{\mathbb{R}^{3}} \frac{\left|\psi\left(x^{\prime}\right)\right|^{2}}{\left|x-x^{\prime}\right|} d x^{\prime}+\Lambda\right) \psi(x)=0, \quad x \in \mathbb{R}^{3}, \\
\|\psi\|_{L^{2}}=1,
\end{gathered}
$$

где $\Delta$ - оператор Лапласа, $\varepsilon>0$ - малый параметр и $a, \alpha$ - положительные константы.

В этой части работы для задачи (1.1), (1.2) построена серия асимптотических собственных значений $\Lambda=\Lambda_{n, m}(\varepsilon), \varepsilon \rightarrow 0$, и соответствуюших собственных функций $\psi=\psi_{n, m}$, носителями которых по $\bmod O\left(\varepsilon^{\infty}\right)$ являются плоские диски

$$
D=\left\{(z, \rho, \varphi) \mid z=0, \rho_{-} \leqslant \rho \leqslant \rho_{+}\right\} .
$$

Здесь $(z, \rho, \varphi)$ - цилиндрические координаты в $\mathbb{R}^{3}$.

Подчеркнем, что функции $\psi_{n, m}$ заведомо не являются радиально-симметричными. Хотя подобные локализованные в дисках решения хорошо известны в линейных задачах, для уравнения Хартри их построение является серьезной проблемой. Здесь до сих пор были известны лишь радиально-симметричные асимптотики [2], [3]. Доказательство сушествования несимметричных решений уравнения

(C) M. B. Kapaceb, А. В. Перескоков, 2001 
Хартри для некоторых случаев дано в [4], [5]. Кроме того, изучение нерадиально-симметричных решений производилось с использованием численных методов (см., например, [6]). Более подробно история вопроса изложена во введении к первой части [1]. Там же приведен большой список литературы.

Ниже мы построим асимптотические решения задачи (1.1), (1.2) в случае, когда число $\Lambda$ велико и имеет порядок $\varepsilon^{-\alpha \omega}$, где

$$
\omega=\frac{2}{4+5 \alpha}>0
$$

Именно в этом случае задача (1.1), (1.2) приводит к рассмотренному в [1] модельному уравнению

$$
\frac{\partial^{2} G}{\partial \xi^{2}}+\frac{\partial^{2} G}{\partial \eta^{2}}+\left(\xi-\iint_{-\infty}^{\infty} \ln \left[\frac{\left(\xi-\xi^{\prime}\right)^{2}+\left(\eta-\eta^{\prime}\right)^{2}}{\left(\xi^{\prime}\right)^{2}}\right] G^{2}\left(\xi^{\prime}, \eta^{\prime}\right) d \xi^{\prime} d \eta^{\prime}\right) G=0
$$

Это - так называемая эйри-версия двумерного уравнения полярона, содержащая одновременно как линейное слагаемое $\xi G$, так и интегральную нелинейность типа Хартри.

Отметим, что если при $\varepsilon \rightarrow 0 \Lambda=O(1)$ или $\Lambda$ растет медленнее, чем $\varepsilon^{-\alpha \omega}$, то нелинейность в уравнении (1.1) оказывается очень сильной и линейный потенциал уже не будет входить в модельное уравнение. В этом случае построение нерадиально-симметричной асимптотики решения задачи (1.1), (1.2) остается открытой проблемой.

Наоборот, если при $\varepsilon \rightarrow 0$ числа $\Lambda$ растут быстрее, чем $\varepsilon^{-\alpha \omega}$, то влияние нелинейности в (1.1) слабое; в частности, нелинейное слагаемое вообще не войдет в модельное уравнение.

Таким образом, изучаемая в данной работе зона $\Lambda \sim e^{-\alpha \omega}$ является пограничной между сильно нелинейной зоной (где внешний потенциал не влияет на модельную задачу) и зоной слабой нелинейности (где вообще не будет вклада нелинейности в модельную задачу).

Решения (1.1) мы будем искать в виде

$$
\psi=e^{i M \varphi / \varepsilon^{\nu}} p(\rho, z) / \sqrt{2 \pi \rho}
$$

Здесь

$$
\nu=\frac{6+6 \alpha}{4+5 \alpha}>0,
$$

а $M=M(\varepsilon)$ удовлетворяет неравенствам

$$
0<c_{0} \leqslant M \leqslant c_{1},
$$

где $c_{0}, c_{1}$ - некоторые константы. В силу $2 \pi$-периодичности решения по $\varphi$ константа $M$ должна иметь вид $M=m \varepsilon^{\nu}$, где $m$ - целое (при $\varepsilon \rightarrow 0$ число $m$ велико: $\left.m \sim \varepsilon^{-1 / \nu}\right)$. 
После подстановки (1.5) в (1.1), (1.2) получается следующая задача для функции $p$ :

$$
\begin{gathered}
\varepsilon^{2}\left(\frac{\partial^{2} p}{\partial \rho^{2}}+\frac{\partial^{2} p}{\partial z^{2}}\right)+\left\{-\frac{\varepsilon^{2-2 \nu}}{\rho^{2}} M^{2}-a\left(\rho^{2}+z^{2}\right)^{\alpha / 2}+\frac{\varepsilon^{2}}{4 \rho^{2}}\right. \\
\left.+\Lambda+\int_{0}^{\infty} \int_{-\infty}^{\infty} W\left(\rho, \rho^{\prime}, z, z^{\prime}\right) p^{2}\left(\rho^{\prime}, z^{\prime}\right) d z^{\prime} d \rho^{\prime}\right\} p=0 \\
\int_{0}^{\infty} \int_{-\infty}^{\infty} p^{2}\left(\rho^{\prime}, z^{\prime}\right) d z^{\prime} d \rho^{\prime}=1 .
\end{gathered}
$$

Здесь ядро интегрального оператора имеет вид

$$
W=\frac{1}{2 \pi} \int_{0}^{2 \pi} \frac{d \varphi}{\sqrt{\left(z-z^{\prime}\right)^{2}+\rho^{2}+\left(\rho^{\prime}\right)^{2}-2 \rho \rho^{\prime} \cos \varphi}} .
$$

Функция $W$ выражается через полный эллиптический интеграл первого рода

$$
K(\mathbb{k})=\int_{0}^{\pi / 2} \frac{d \varphi}{\sqrt{1-\mathbb{k}^{2} \sin ^{2} \varphi}}
$$

следуюшим образом:

$$
W=\frac{2}{\pi \sqrt{\left(z-z^{\prime}\right)^{2}+\left(\rho-\rho^{\prime}\right)^{2}}} K\left(\frac{2 i \sqrt{\rho \rho^{\prime}}}{\sqrt{\left(z-z^{\prime}\right)^{2}+\left(\rho-\rho^{\prime}\right)^{2}}}\right) .
$$

Используя тождество [7]

$$
K(\mathbb{k})=\frac{1}{\sqrt{1-\mathbb{k}^{2}}} K\left(-\frac{i \mathbb{k}}{\sqrt{1-\mathbb{k}^{2}}}\right),
$$

где модуль $\mathbb{k}=2 i \sqrt{\rho \rho^{\prime}} / \sqrt{\left(z-z^{\prime}\right)^{2}+\left(\rho-\rho^{\prime}\right)^{2}}$, получаем следующее представление для интегрального ядра:

$$
W\left(\rho, \rho^{\prime}, z, z^{\prime}\right)=\frac{2}{\pi \sqrt{\left(z-z^{\prime}\right)^{2}+\left(\rho+\rho^{\prime}\right)^{2}}} K\left(\frac{2 \sqrt{\rho \rho^{\prime}}}{\sqrt{\left(z-z^{\prime}\right)^{2}+\left(\rho+\rho^{\prime}\right)^{2}}}\right) .
$$

Наконец, по соображениям симметрии относительно плоскости $z=0$ дополним (1.7), (1.8) условием

$$
\int_{0}^{\infty} \int_{-\infty}^{\infty} z^{\prime} p^{2}\left(\rho^{\prime}, z^{\prime}\right) d z^{\prime} d \rho^{\prime}=0
$$

Пусть $\Lambda=\lambda \varepsilon^{-\omega \alpha}, m=M \varepsilon^{-\nu}$, где $M$ удовлетворяет (1.6), a $0 \leqslant c_{2}<\lambda(\varepsilon) \leqslant c_{3}$, где $c_{2}, c_{3}$ - некоторые константы. Тогда после замены

$$
x=\rho \varepsilon^{\omega}, \quad y=z \varepsilon^{\omega}, \quad g=p \varepsilon^{-\omega}, \quad h=\varepsilon^{\nu}
$$


задача (1.7), (1.8), (1.10) принимает вид

$$
\begin{gathered}
\left\{h^{2}\left(\frac{\partial^{2}}{\partial x^{2}}+\frac{\partial^{2}}{\partial y^{2}}\right)-\frac{M^{2}}{x^{2}}-a\left(x^{2}+y^{2}\right)^{\alpha / 2}+\lambda+\frac{h^{2}}{4 x^{2}}\right. \\
\left.+h^{1 / 3} \int_{0}^{\infty} \int_{-\infty}^{\infty} W\left(x, x^{\prime}, y, y^{\prime}\right) g^{2}\left(x^{\prime}, y^{\prime}\right) d y^{\prime} d x^{\prime}\right\} g(x, y)=0 \\
\int_{0}^{\infty} \int_{-\infty}^{\infty} g^{2}\left(x^{\prime}, y^{\prime}\right) d y^{\prime} d x^{\prime}=1 \\
\int_{0}^{\infty} \int_{-\infty}^{\infty} y^{\prime} g^{2}\left(x^{\prime}, y^{\prime}\right) d y^{\prime} d x^{\prime}=0
\end{gathered}
$$

где ядро $W$ задается формулой $(1.9), h \rightarrow 0$.

Так как перед интегралом в (1.11) стоит мальй параметр $h^{1 / 3}$, то нелинейность в (1.11) достаточно слабая.

Введем функцию $V(x) \stackrel{\text { def }}{=} M^{2} / x^{2}+a x^{\alpha}$. Поскольку $a>0, \alpha>0$, то функция $V(x)$ имеет единственную точку минимума

$$
x_{0}=\left(\frac{2 M^{2}}{\alpha a}\right)^{1 /(\alpha+2)} .
$$

Пусть ниже $\lambda>V\left(x_{0}\right)$. Тогда функция

$$
U(x) \stackrel{\text { def }}{=} \lambda-V(x)
$$

имеет ровно две точки $x_{-}, x_{+}, x_{-}<x_{+}$, в которых $U\left(x_{ \pm}\right)=0$, причем $U(x)>0$ при $x \in\left(x_{-}, x_{+}\right)$и $U(x)<0$ при $0<x<x_{-}, x>x_{+}$. Таким образом, $-U(x)$ является потенциальной ямой.

Асимптотические решения уравнения (1.11) будем строить, следуя общей идеологии согласования асимптотических разложений [8]. В области между точками $x_{-}, x_{+}$для нахождения асимптотики используется вариант метода ВКБ. Вблизи же от точек $\left(x_{-}, 0\right),\left(x_{+}, 0\right)$ асимптотика выражается через решения модельного уравнения. Именно в том случае, когда в (1.11) перед интегралом стоит множитель $h^{1 / 3}$, модельным является уравнение (1.4); его экспоненциально убываюшие при $\eta \rightarrow \pm \infty, \xi \rightarrow-\infty$ решения (эйри-поляроны) были изучены в [1].

Ниже, зафиксировав $M$, для нахождения дискретной серии асимптотических собственных значений задачи (1.11)-(1.13) $\lambda=\lambda_{n}(h)$ выпишем аналог правила квантования типа Бора-Зоммерфельда (см. ниже (6.9)). В это правило войдет определяющая фазу ВКБ-разложения функция $S(x, h)$, задача для которой имеет достаточно сложный вид (см. (5.7), (5.12)-(5.15), (5.23)).

Ее решение приводит к громоздким формулам. Ограничимся нахождением $\lambda_{n}(h)=O(1)$ лишь с точностью $O\left(n^{-7 / 10}\right)$ при $h \rightarrow 0, n \sim 1 / h$ (главное приближение). Тогда правило квантования (6.9) упростится и приведет к следуюшему 
алгебраическому уравнению для чисел $\lambda=\lambda_{n}(h)$ :

$$
\begin{aligned}
\frac{1}{h} \int_{x_{-}}^{x_{+}} & \sqrt{U(x)} d x+\frac{k_{0}}{2 h^{2 / 3}} \int_{x_{-}}^{x_{+}} \frac{\Omega(x)}{\sqrt{U(x)}} d x \\
& +\frac{1}{h^{1 / 3}}\left\{\frac{k_{0}^{3} P_{1}}{8} \int_{x_{-}}^{x_{+}} \frac{\Omega(x)}{\sqrt{U(x)}} d x-\frac{k_{0}^{2} P_{2}}{4}-\frac{k_{0}^{2} P_{3}}{8}+P\right\}=\pi n+O\left(n^{1 / 3-1 / 30}\right) .
\end{aligned}
$$

Неизвестное $\lambda$ входит в это уравнение через функцию $U(1.14)$ и через точки поворота $x_{ \pm}$, в которых $U\left(x_{ \pm}\right)=0$.

В уравнении (1.15) $n$ - целые числа, $n \rightarrow \infty$ (так что выполнены неравенства $(6.10))$, а коэффициенты $k_{0}, P_{j}, j=1,2,3$, и функция $\Omega(x)$ зависят от $U$ следующим образом:

$$
\begin{aligned}
& k_{0}=2\left(\int_{x_{-}}^{x_{+}} \frac{d x}{\sqrt{U(x)}}\right)^{-1} \\
& \Omega(x)=\int_{x_{-}}^{x_{+}} \frac{1}{\pi\left(x+x^{\prime}\right)} K\left(\frac{2 \sqrt{x x^{\prime}}}{x+x^{\prime}}\right) \frac{d x^{\prime}}{\sqrt{U\left(x^{\prime}\right)}}, \\
& P_{1}=\int_{-\infty}^{\infty}\left\{\frac{\theta\left(x-x_{-}\right) \theta\left(x_{+}-x\right)}{(U(x))^{3 / 2}} \Omega(x)-\frac{\theta\left(x-x_{-}\right) \Omega\left(x_{-}\right)}{\left(U^{\prime}\left(x_{-}\right)\right)^{3 / 2}\left(x-x_{-}\right)^{3 / 2}}\right. \\
& \left.-\frac{\theta\left(x_{+}-x\right) \Omega\left(x_{+}\right)}{\left(-U^{\prime}\left(x_{+}\right)\right)^{3 / 2}\left(x_{+}-x\right)^{3 / 2}}\right\} d x, \\
& P_{2}=\int_{-\infty}^{\infty} \Omega(x)\left\{\frac{\theta\left(x-x_{-}\right) \theta\left(x_{+}-x\right)}{(U(x))^{3 / 2}} \Omega(x)-\frac{\theta\left(x-x_{-}\right) \Omega\left(x_{-}\right)}{\left(U^{\prime}\left(x_{-}\right)\right)^{3 / 2}\left(x-x_{-}\right)^{3 / 2}}\right. \\
& \left.-\frac{\theta\left(x_{+}-x\right) \Omega\left(x_{+}\right)}{\left(-U^{\prime}\left(x_{+}\right)\right)^{3 / 2}\left(x_{+}-x\right)^{3 / 2}}\right\} d x, \\
& P_{3}=\int_{-\infty}^{\infty}\left\{\frac{\theta\left(x-x_{-}\right) \theta\left(x_{+}-x\right)}{(U(x))^{3 / 2}} \Omega^{2}(x)-\frac{\theta\left(x-x_{-}\right) \Omega^{2}\left(x_{-}\right)}{\left(U^{\prime}\left(x_{-}\right)\right)^{3 / 2}\left(x-x_{-}\right)^{3 / 2}}\right. \\
& \left.-\frac{\theta\left(x_{+}-x\right) \Omega^{2}\left(x_{+}\right)}{\left(-U^{\prime}\left(x_{+}\right)\right)^{3 / 2}\left(x_{+}-x\right)^{3 / 2}}\right\} d x,
\end{aligned}
$$

где

а также

$$
\theta(x)=\left\{\begin{array}{lll}
1 & \text { при } x>0, \\
0 & \text { при } x \leqslant 0,
\end{array}\right.
$$

$$
\begin{aligned}
P= & \frac{1}{2} \int_{x_{-}}^{x_{+}} \frac{1}{\sqrt{U(x)}}\left\{-k_{0}\left(b_{-}+b_{+}\right) \Omega(x)+\frac{2}{\pi}\left[\frac{b_{-}}{x+x_{-}} K\left(\frac{2 \sqrt{x x_{-}}}{x+x_{-}}\right)\right.\right. \\
& \left.\left.+\frac{b_{+}}{x+x_{+}} K\left(\frac{2 \sqrt{x x_{+}}}{x+x_{+}}\right)\right]\right\} d x,
\end{aligned}
$$

где

$$
\begin{aligned}
& b_{-}=\left(U^{\prime}\left(x_{-}\right)\right)^{2 / 3} 2 \pi x_{-} A_{-1}\left(\frac{k_{0}}{2 \pi x_{-}\left(U^{\prime}\left(x_{-}\right)\right)^{4 / 3}}\right) \\
& b_{+}=\left(-U^{\prime}\left(x_{+}\right)\right)^{2 / 3} 2 \pi x_{+} A_{-1}\left(\frac{k_{0}}{2 \pi x_{+}\left(-U^{\prime}\left(x_{+}\right)\right)^{4 / 3}}\right) .
\end{aligned}
$$


Функция $A_{-1}=A_{-1}(\tilde{k})$ в $(1.22)$, характеризующая влияние решений модельного уравнения (1.4) на правило квантования, а значит, и на спектр исходной задачи, определена формулой (4.21).

Отметим, что уравнение (1.15) содержит негладкую функцию $\Omega(x)$ и вывод правила квантования (1.15) требует очень скрупулезных оценок (см. ниже $\S 7,8)$.

\section{§ 2. ВКБ-асимптотика. Уравнения для амплитуды и фазы}

Будем строить асимптотические решения $g$ в виде

$$
g(x, y)=h^{-5 / 18} T\left(x, \tau_{1}, h\right) \cos \Phi\left(x, \tau_{1}, h\right)
$$

где

$$
\tau_{1}=\frac{y}{h^{5 / 9}}, \quad \Phi\left(x, \tau_{1}, h\right)=\frac{S(x, h)}{h}+h^{1 / 9} \varphi\left(x, \tau_{1}, h\right) .
$$

Функции $S, T, \varphi$ в $(2.1),(2.2)$ гладкие, вещественные; при $\tau_{1} \rightarrow \pm \infty$ функция $T$ экспоненциально убывает, а $\varphi$ имеет степенной рост. Для упрощения обозначений мы не будем явно указывать зависимость $g$ от параметра $h$.

Формулы (2.1), (2.2) справедливы при

$$
\tilde{x}_{-}+\varepsilon<x<\tilde{x}_{+}-\varepsilon
$$

Здесь точки $\tilde{x}_{ \pm} \sim x_{ \pm}$определяются из уравнения

$$
\begin{aligned}
U\left(\tilde{x}_{ \pm}\right) & +h^{1 / 3} \int_{0}^{\infty} \frac{2}{\pi\left(\tilde{x}_{ \pm}+x^{\prime}\right)} K\left(\frac{2 \sqrt{\tilde{x}_{ \pm} x^{\prime}}}{\tilde{x}_{ \pm}+x^{\prime}}\right) \int_{-\infty}^{\infty} g^{2}\left(x^{\prime}, y^{\prime}\right) d y^{\prime} d x^{\prime} \\
& =O\left(h^{1+1 / 57} \ln \frac{1}{h}\right), \quad h \rightarrow 0
\end{aligned}
$$

$\varepsilon=c h^{26 / 57}$, где $c>0-$ произвольная константа.

В настоящем параграфе мы получим уравнения для амплитуды $T$ и функций $S$ и $\varphi$, задающих фазу (2.1). Поскольку построение ВКБ-асимптотики для (1.11) аналогично [1], метод ВКБ будет изложен кратко.

Так как решения $g$ по $\bmod O\left(h^{\infty}\right)$ локализованы вблизи $y=0$, разложим заданное соотношением (1.9) ядро $W\left(x, x^{\prime}, y, y^{\prime}\right)$ по степеням $y-y^{\prime}$.

ЛЕмма 2.1. При $у-y^{\prime} \rightarrow 0$ справедливо равенство

$$
\begin{aligned}
W\left(x, x^{\prime}, y, y^{\prime}\right)= & \frac{2}{\pi\left(x+x^{\prime}\right)} K\left(\frac{2 \sqrt{x x^{\prime}}}{x+x^{\prime}}\right)-\frac{1}{\pi\left(x+x^{\prime}\right)} \ln \left(1+\frac{\left(y-y^{\prime}\right)^{2}}{\left(x-x^{\prime}\right)^{2}}\right) \\
& +O\left(\frac{\left(y-y^{\prime}\right)^{2}}{\left(x+x^{\prime}\right)^{3}}\left(1+|\ln | x-x^{\prime}||\right)\right) .
\end{aligned}
$$


ДоКАЗАТЕЛЬСТВО. Поскольку функция $K(\mathbb{k})$ имеет логарифмическую особенность при $\mathbb{k} \rightarrow 1[7]:$

$$
K(\mathbb{k})=\ln \left(4 / \sqrt{1-\mathbb{k}^{2}}\right)+O\left(\left(1-\mathbb{k}^{2}\right) \ln \left(1-\mathbb{k}^{2}\right)\right),
$$

то непосредственно к $W$ формула Тейлора не применима. Поэтому представим

$$
W\left(x, x^{\prime}, y, y^{\prime}\right)=\frac{2}{\pi\left(x+x^{\prime}\right)}\left[K\left(\frac{2 \sqrt{x x^{\prime}}}{x+x^{\prime}}\right)+\delta K\right]+O\left(\frac{\left(y-y^{\prime}\right)^{2}}{\left(x+x^{\prime}\right)^{3}} K\left(\frac{2 \sqrt{x x^{\prime}}}{x+x^{\prime}}\right)\right),
$$

где

$$
\delta K=K\left(\frac{2 \sqrt{x x^{\prime}}}{\sqrt{\left(y-y^{\prime}\right)^{2}+\left(x+x^{\prime}\right)^{2}}}\right)-K\left(\frac{2 \sqrt{x x^{\prime}}}{x+x^{\prime}}\right) .
$$

Для дальнейшего разложения $\delta K$ воспользуемся равенством [7]

$$
\frac{d K}{d b}=\frac{1}{2 b(1-b)} E-\frac{1}{2 b} K,
$$

где $b=\mathbb{k}^{2}$, a $E(\mathbb{k})$ - полный эллиптический интеграл второго рода. Учитывая асимптотику $E(\mathbb{k})=1+O\left(\left(1-\mathbb{k}^{2}\right) \ln \left(1-\mathbb{k}^{2}\right)\right), \mathbb{k} \rightarrow 1$, а также (2.6), имеем

$$
\begin{aligned}
\delta K & =\int_{4 x x^{\prime} /\left(x+x^{\prime}\right)^{2}}^{4 x x^{\prime} /\left[\left(y-y^{\prime}\right)^{2}+\left(x+x^{\prime}\right)^{2}\right]}\left\{\frac{1}{2 b(1-b)} E(\sqrt{b})-\frac{1}{2 b} K(\sqrt{b})\right\} d b \\
& =-\frac{1}{2} \ln \left(1+\frac{\left(y-y^{\prime}\right)^{2}}{\left(x-x^{\prime}\right)^{2}}\right)+O\left(\frac{\left(y-y^{\prime}\right)^{2}}{\left(x+x^{\prime}\right)^{2}}\left(1+|\ln | x-x^{\prime}||\right)\right) .
\end{aligned}
$$

Остается подставить (2.9) в (2.7). Приходим к формуле (2.5). Лемма доказана.

В силу (1.11), (2.5), а также равенства $\left(x^{2}+y^{2}\right)^{\alpha / 2}=x^{\alpha}+O\left(y^{2} x^{\alpha-2}\right), y \rightarrow 0$, получаем

$$
\begin{aligned}
& h^{2}\left(\frac{\partial^{2} g}{\partial x^{2}}+\frac{\partial^{2} g}{\partial y^{2}}\right)+\left\{U(x)+h^{1 / 3} \int_{0}^{\infty} \frac{2}{\pi\left(x+x^{\prime}\right)} K\left(\frac{2 \sqrt{x x^{\prime}}}{x+x^{\prime}}\right) \int_{-\infty}^{\infty} g^{2}\left(x^{\prime}, y^{\prime}\right) d y^{\prime} d x^{\prime}\right. \\
& \quad-h^{1 / 3} \int_{0}^{\infty} \frac{1}{\pi\left(x+x^{\prime}\right)} \int_{-\infty}^{\infty} \ln \left(1+\frac{\left(y-y^{\prime}\right)^{2}}{\left(x-x^{\prime}\right)^{2}}\right) g^{2}\left(x^{\prime}, y^{\prime}\right) d y^{\prime} d x^{\prime} \\
& \quad+O\left(h^{2} / x^{2}\right)+O\left(y^{2} x^{\alpha-2}\right) \\
& \left.\quad+O\left(h^{1 / 3} \int_{0}^{\infty} \int_{-\infty}^{\infty} \frac{\left(y-y^{\prime}\right)^{2}}{\left(x+x^{\prime}\right)^{3}}\left(1+|\ln | x-x^{\prime}||\right) g^{2}\left(x^{\prime}, y^{\prime}\right) d y^{\prime} d x^{\prime}\right)\right\} g=0 .
\end{aligned}
$$


Здесь $U(x)$ задано (1.14). Подставим затем асимптотическое решение (2.1), (2.2) в уравнение (2.10). Имеем

$$
\begin{aligned}
h^{2} \frac{\partial^{2} g}{\partial x^{2}}= & \frac{1}{h^{5 / 18}}\left\{-\left[T\left(S^{\prime}\right)^{2}+2 h^{10 / 9} T S^{\prime} \frac{\partial \varphi}{\partial x}\right] \cos \Phi\right. \\
& -h\left[2 \frac{\partial T}{\partial x} S^{\prime}+T S^{\prime \prime}\right] \sin \Phi+O\left(h^{2} \frac{\partial^{2} T}{\partial x^{2}}\right)+O\left(h^{20 / 9} T\left(\frac{\partial \varphi}{\partial x}\right)^{2}\right) \\
& \left.+O\left(h^{19 / 9} \frac{\partial T}{\partial x} \frac{\partial \varphi}{\partial x}\right)+O\left(h^{19 / 9} T \frac{\partial^{2} \varphi}{\partial x^{2}}\right)\right\} \\
h^{2} \frac{\partial^{2} g}{\partial y^{2}}= & \frac{1}{h^{5 / 18}}\left\{\left[h^{8 / 9} \frac{\partial^{2} T}{\partial \tau_{1}^{2}}-h^{10 / 9} T\left(\frac{\partial \varphi}{\partial \tau_{1}}\right)^{2}\right] \cos \Phi\right. \\
& \left.-h\left[2 \frac{\partial T}{\partial \tau_{1}} \frac{\partial \varphi}{\partial \tau_{1}}+T \frac{\partial^{2} \varphi}{\partial \tau_{1}^{2}}\right] \sin \Phi\right\} .
\end{aligned}
$$

Пусть $\chi=\chi(\xi) \in C^{\infty}\left(\mathbb{R}^{1}\right)$ - неотрицательная функция такая, что $\chi(\xi) \equiv 1$ при $\xi<1 / 2$ и $\chi(\xi) \equiv 0$ при $\xi>1$. Определим

$$
\chi_{-}(x, \varepsilon) \stackrel{\text { def }}{=} \chi\left(\left(x-\tilde{x}_{-}\right) / \varepsilon\right), \quad \chi_{+}(x, \varepsilon) \stackrel{\text { def }}{=} \chi\left(\left(\tilde{x}_{+}-x\right) / \varepsilon\right) .
$$

Так как $\varepsilon$ содержит произвольную константу $c$, ВКБ-приближение (2.1) будет справедливо и в более широкой, чем (2.3), области $x \in\left(\tilde{x}_{-}+\varepsilon / 2, \tilde{x}_{+}-\varepsilon / 2\right)$. Предполагая, что вблизи точек $\tilde{x}_{-}, \tilde{x}_{+}$точное решение $g$ известно, получаем [1]

$$
\begin{aligned}
h^{1 / 3}\{ & \int_{0}^{\infty} \frac{2}{\pi\left(x+x^{\prime}\right)} K\left(\frac{2 \sqrt{x x^{\prime}}}{x+x^{\prime}}\right) \int_{-\infty}^{\infty} g^{2}\left(x^{\prime}, y^{\prime}\right) d y^{\prime} d x^{\prime} \\
& \left.-\int_{0}^{\infty} \frac{1}{\pi\left(x+x^{\prime}\right)} \int_{-\infty}^{\infty} \ln \left(1+\frac{\left(y-y^{\prime}\right)^{2}}{\left(x-x^{\prime}\right)^{2}}\right) g^{2}\left(x^{\prime}, y^{\prime}\right) d y^{\prime} d x^{\prime}\right\} T\left(x, \tau_{1}, h\right) \\
= & h^{1 / 3}\left\{j_{-}(x, h)+\int_{\tilde{x}_{-}+\varepsilon / 2}^{\tilde{x}_{+}-\varepsilon / 2} \frac{1}{\pi\left(x+x^{\prime}\right)} K\left(\frac{2 \sqrt{x x^{\prime}}}{x+x^{\prime}}\right)\left(1-\chi_{-}\left(x^{\prime}, \varepsilon\right)-\chi_{+}\left(x^{\prime}, \varepsilon\right)\right)\right. \\
& \left.\times \int_{-\infty}^{\infty} T^{2}\left(x^{\prime}, \tau_{1}^{\prime}, h\right) d \tau_{1}^{\prime} d x^{\prime}+j_{+}(x, h)\right\} T\left(x, \tau_{1}, h\right) \\
& -\frac{h^{8 / 9}}{2 x} \int_{-\infty}^{\infty}\left|\tau_{1}-\tau_{1}^{\prime}\right| T^{2}\left(x, \tau_{1}^{\prime}, h\right) d \tau_{1}^{\prime} T\left(x, \tau_{1}, h\right)+r .
\end{aligned}
$$

Здесь $x \in\left(\tilde{x}_{-}+\varepsilon, \tilde{x}_{+}-\varepsilon\right)$,

$$
\begin{aligned}
& j_{-}(x, h) \stackrel{\text { def }}{=} \int_{0}^{\tilde{x}_{-}+\varepsilon} \frac{2}{\pi\left(x+x^{\prime}\right)} K\left(\frac{2 \sqrt{x x^{\prime}}}{x+x^{\prime}}\right) \chi_{-}\left(x^{\prime}, \varepsilon\right) \int_{-\infty}^{\infty} g^{2}\left(x^{\prime}, y^{\prime}\right) d y^{\prime} d x^{\prime}, \\
& j_{+}(x, h) \stackrel{\text { def }}{=} \int_{\tilde{x}_{+}-\varepsilon}^{\infty} \frac{2}{\pi\left(x+x^{\prime}\right)} K\left(\frac{2 \sqrt{x x^{\prime}}}{x+x^{\prime}}\right) \chi_{+}\left(x^{\prime}, \varepsilon\right) \int_{-\infty}^{\infty} g^{2}\left(x^{\prime}, y^{\prime}\right) d y^{\prime} d x^{\prime},
\end{aligned}
$$


а остаточный член $r$ допускает оценку

$$
\begin{aligned}
r= & O\left(h ^ { 1 3 / 9 } \int _ { - \infty } ^ { \infty } \mathrm { v } \cdot \mathrm { p } \cdot \int _ { - \infty } ^ { \infty } \frac { ( \tau _ { 1 } - \tau _ { 1 } ^ { \prime } ) ^ { 2 } } { ( x - x ^ { \prime } ) ^ { 2 } } \left[\left(1-\chi_{-}\left(x^{\prime}, \varepsilon\right)-\chi_{+}\left(x^{\prime}, \varepsilon\right)\right) \frac{T^{2}\left(x^{\prime}, \tau_{1}^{\prime}, h\right)}{x+x^{\prime}}\right.\right. \\
& \left.\left.-\frac{1}{2 x} T^{2}\left(x, \tau_{1}^{\prime}, h\right)\right] d x^{\prime} d \tau_{1}^{\prime} T\left(x, \tau_{1}, h\right)\right) \\
& +O\left(h^{1 / 3} \int_{0}^{\tilde{x}_{-}+\varepsilon} \frac{1}{\left(x+x^{\prime}\right)} \int_{-\infty}^{\infty} \frac{\left(y-y^{\prime}\right)^{2}}{\left(x-x^{\prime}\right)^{2}} \chi_{-}\left(x^{\prime}, \varepsilon\right) g^{2}\left(x^{\prime}, y^{\prime}\right) d y^{\prime} d x^{\prime} T\left(x, \tau_{1}, h\right)\right) \\
& +O\left(h^{1 / 3} \int_{\tilde{x}_{+}-\varepsilon}^{\infty} \frac{1}{\left(x+x^{\prime}\right)} \int_{-\infty}^{\infty} \frac{\left(y-y^{\prime}\right)^{2}}{\left(x-x^{\prime}\right)^{2}} \chi_{+}\left(x^{\prime}, \varepsilon\right) g^{2}\left(x^{\prime}, y^{\prime}\right) d y^{\prime} d x^{\prime} T\left(x, \tau_{1}, h\right)\right) \\
& +O\left(h^{1 / 3} \int_{\tilde{x}_{-}+\varepsilon / 2}^{\tilde{x}_{+}-\varepsilon / 2} \int_{-\infty}^{\infty} \frac{1}{\left(x+x^{\prime}\right)}\left[2 K\left(\frac{2 \sqrt{x x^{\prime}}}{x+x^{\prime}}\right)-\ln \left(1+h^{10 / 9} \frac{\left(\tau_{1}-\tau_{1}^{\prime}\right)^{2}}{\left(x-x^{\prime}\right)^{2}}\right)\right]\right. \\
& \times\left(1-\chi-\left(x^{\prime}, \varepsilon\right)-\chi_{+}\left(x^{\prime}, \varepsilon\right)\right) T^{2}\left(x^{\prime}, \tau_{1}^{\prime}, h\right) \\
& \left.\times \cos 2 \Phi\left(x^{\prime}, \tau_{1}^{\prime}, h\right) d \tau_{1}^{\prime} d x^{\prime} T\left(x, \tau_{1}, h\right)\right) .
\end{aligned}
$$

Наконец, подставляя (2.11)-(2.13) в (2.10), получаем следующую лемму.

ЛЕмма 2.2. При $x \in\left(\tilde{x}_{-}+\varepsilon, \tilde{x}_{+}-\varepsilon\right)$ справедливо равенство

$$
\begin{aligned}
& -\left[\left(S^{\prime}\right)^{2} T-h^{8 / 9} \frac{\partial^{2} T}{\partial \tau_{1}^{2}}+2 h^{10 / 9} T S^{\prime} \frac{\partial \varphi}{\partial x}+h^{10 / 9} T\left(\frac{\partial \varphi}{\partial \tau_{1}}\right)^{2}\right] \cos \Phi \\
& \quad-h\left[2 \frac{\partial T}{\partial \tau_{1}} \frac{\partial \varphi}{\partial \tau_{1}}+T \frac{\partial^{2} \varphi}{\partial \tau_{1}^{2}}+2 \frac{\partial T}{\partial x} S^{\prime}+T S^{\prime \prime}\right] \sin \Phi \\
& \quad+\left\{U+h^{1 / 3}\left[j_{-}+\int_{\tilde{x}_{-}+\varepsilon / 2}^{\tilde{x}_{+}-\varepsilon / 2} \frac{1}{\pi\left(x+x^{\prime}\right)} K\left(\frac{2 \sqrt{x x^{\prime}}}{x+x^{\prime}}\right)\right.\right. \\
& \left.\quad \times\left(1-\chi_{-}\left(x^{\prime}, \varepsilon\right)-\chi_{+}\left(x^{\prime}, \varepsilon\right)\right) \int_{-\infty}^{\infty} T^{2}\left(x^{\prime}, \tau_{1}^{\prime}, h\right) d \tau_{1}^{\prime} d x^{\prime}+j_{+}\right] \\
& \left.\quad-\frac{h^{8 / 9}}{2 x} \int_{-\infty}^{\infty}\left|\tau_{1}-\tau_{1}^{\prime}\right| T^{2}\left(x, \tau_{1}^{\prime}, h\right) d \tau_{1}^{\prime}\right\} T \cos \Phi+r^{*}=0
\end{aligned}
$$

$2 \partial e$

$$
\begin{aligned}
r^{*}= & \left(h^{2} \frac{\partial^{2} T}{\partial x^{2}}\right)+O\left(h^{19 / 9} \frac{\partial T}{\partial x} \frac{\partial \varphi}{\partial x}\right)+O\left(h^{19 / 9} T \frac{\partial \varphi^{2}}{\partial x^{2}}\right) \\
& +O\left(h^{20 / 9} T\left(\frac{\partial \varphi}{\partial x}\right)^{2}\right)+O(r)+O\left(h^{10 / 9} \tau_{1}^{2} T\right)+O\left(h^{2} T\right) \\
& +O\left(h^{13 / 9} \int_{\tilde{x}_{-}+\varepsilon / 2}^{\tilde{x}_{+}-\varepsilon / 2} \int_{-\infty}^{\infty}\left(\tau_{1}^{2}+\left(\tau_{1}^{\prime}\right)^{2}\right)\left(1+|\ln | x-x^{\prime}||\right)\right. \\
& \left.\times\left(1-\chi_{-}\left(x^{\prime}, \varepsilon\right)-\chi_{+}\left(x^{\prime}, \varepsilon\right)\right) T^{2}\left(x^{\prime}, \tau_{1}^{\prime}, h\right) d \tau_{1}^{\prime} d x^{\prime} T\left(x, \tau_{1}, h\right)\right)
\end{aligned}
$$




$$
\begin{aligned}
& +O\left(h^{1 / 3} \int_{0}^{\tilde{x}_{-}+\varepsilon} \int_{-\infty}^{\infty}\left(y-y^{\prime}\right)^{2}\right. \\
& \left.\times\left(1+|\ln | x-x^{\prime}||\right) \chi_{-}\left(x^{\prime}, \varepsilon\right) g^{2}\left(x^{\prime}, y^{\prime}\right) d y^{\prime} d x^{\prime} T\left(x, \tau_{1}, h\right)\right) \\
& +O\left(h^{1 / 3} \int_{\tilde{x}_{+}-\varepsilon}^{\infty} \int_{-\infty}^{\infty}\left(y-y^{\prime}\right)^{2}\right. \\
& \left.\times\left(1+|\ln | x-x^{\prime}||\right) \chi_{+}\left(x^{\prime}, \varepsilon\right) g^{2}\left(x^{\prime}, y^{\prime}\right) d y^{\prime} d x^{\prime} T\left(x, \tau_{1}, h\right)\right)
\end{aligned}
$$

Приравняем в (2.16) к нулю выражение перед $\sin \Phi$. Получаем

$$
\frac{\partial}{\partial \tau_{1}}\left(T^{2} \frac{\partial \varphi}{\partial \tau_{1}}\right)+\frac{\partial}{\partial x}\left(T^{2} S^{\prime}\right)=0
$$

Так как при $\left|\tau_{1}\right| \rightarrow \infty T$ экспоненщиально убывает, а $\varphi$ имеет лишь степеной рост, то условием разрешимости (2.18) будет равенство

$$
\int_{-\infty}^{\infty} T^{2}\left(x, \tau_{1}, h\right) d \tau_{1}=\frac{k}{S^{\prime}(x, h)}
$$

где $k$ - константа.

Обратим, далее, в нуль выражение перед, стояшее перед $\cos \Phi$ в выражении (2.16). Пусть $T\left(x, \tau_{1}, h\right)$ и $L(x, h)$ удовлетворяют уравнению

$$
\frac{\partial^{2} T}{\partial \tau_{1}^{2}}-\left(\frac{1}{2 x} \int_{-\infty}^{\infty}\left|\tau_{1}-\tau_{1}^{\prime}\right| T^{2}\left(x, \tau_{1}^{\prime}, h\right) d \tau_{1}^{\prime}+L\right) T=h^{2 / 9}\left[\left(\frac{\partial \varphi}{\partial \tau_{1}}\right)^{2}+2 S^{\prime} \frac{\partial \varphi}{\partial x}\right] T
$$

а также условию (2.19). Тогда получаем следующее уравнение для определения $S(x, h)$ (уравнение для фазы):

$$
\begin{gathered}
-\left(S^{\prime}\right)^{2}+U(x)+h^{1 / 3}\left[\int_{\tilde{x}_{-}+\varepsilon / 2}^{\tilde{x}_{+}-\varepsilon / 2} \frac{1}{\pi\left(x+x^{\prime}\right)} K\left(\frac{2 \sqrt{x x^{\prime}}}{x+x^{\prime}}\right)\right. \\
\quad \times\left(1-\chi_{-}\left(x^{\prime}, \varepsilon\right)-\chi_{+}\left(x^{\prime}, \varepsilon\right)\right) \frac{k}{S^{\prime}\left(x^{\prime}, h\right)} d x^{\prime} \\
\left.+j_{-}(x, h)+j_{+}(x, h)\right]+h^{8 / 9} L(x, h)=0 .
\end{gathered}
$$

Здесь $j_{-}, j_{+}$заданы формулами $(2.14), x \in\left(\tilde{x}_{-}+\varepsilon, \tilde{x}_{+}-\varepsilon\right)$.

Доказана

Теорема 2.1. Пусть функиии $S(x, h), T\left(x, \tau_{1}, h\right), \varphi\left(x, \tau_{1}, h\right), L(x, h)$ удовлетворяют уравнениям (2.18)-(2.21). Тогда при $x \in\left(\tilde{x}_{-}+\varepsilon, \tilde{x}_{+}-\varepsilon\right)$ ВКБ-приближение (2.1), (2.2) является асимптотическим решением уравнения (1.11) с точностью $r^{*}$, где $r^{*}$ имеет вид (2.17). 
ЗАмечАние 2.1. Отметим попутно, что уравнения, подобные (2.21), возникают при изучении контактных задач в теории упругости [9].

Далее определим cos-амплитуду

$$
B=T \cos \left(h^{1 / 9} \varphi\right)
$$

и $\sin$-амплитуду

$$
I=-T \sin \left(h^{1 / 9} \varphi\right),
$$

которые позволяют записать равенство (2.1) в виде

$$
g=\frac{1}{h^{5 / 18}}\left\{B \cos \frac{S}{h}+I \sin \frac{S}{h}\right\}
$$

Оказывается, что технически более удобно строить асимптотические разложения не для пары $T\left(x, \tau_{1}, h\right), \varphi\left(x, \tau_{1}, h\right)$, а для функций $B\left(x, \tau_{1}, h\right), I\left(x, \tau_{1}, h\right)$, через которые выражаются $T$ и $\varphi$.

Пусть $S(x, h)$ удовлетворяет (2.21). Тогда, аналогично [1], получаем следующую задачу для нахождения cos- и sin-амплитуд:

$$
\begin{aligned}
\frac{\partial^{2} B}{\partial \tau_{1}^{2}}- & \left(\frac{1}{2 x} \int_{-\infty}^{\infty}\left|\tau_{1}-\tau_{1}^{\prime}\right|\left(B^{2}\left(x, \tau_{1}^{\prime}, h\right)+I^{2}\left(x, \tau_{1}^{\prime}, h\right)\right) d \tau_{1}^{\prime}+L(x, h)\right) B \\
& +h^{1 / 9}\left(2 S^{\prime} \frac{\partial I}{\partial x}+S^{\prime \prime} I\right)=0 \\
\frac{\partial^{2} I}{\partial \tau_{1}^{2}}- & \left(\frac{1}{2 x} \int_{-\infty}^{\infty}\left|\tau_{1}-\tau_{1}^{\prime}\right|\left(B^{2}\left(x, \tau_{1}^{\prime}, h\right)+I^{2}\left(x, \tau_{1}^{\prime}, h\right)\right) d \tau_{1}^{\prime}+L(x, h)\right) I \\
& -h^{1 / 9}\left(2 S^{\prime} \frac{\partial B}{\partial x}+S^{\prime \prime} B\right)=0, \\
& \int_{-\infty}^{\infty}\left(B^{2}\left(x, \tau_{1}, h\right)+I^{2}\left(x, \tau_{1}, h\right)\right) d \tau_{1}=k / S^{\prime}(x, h) .
\end{aligned}
$$

Теорема 2.2. Пусть функция $S(x, h)$ является решением уравнения (2.21), а функции $B\left(x, \tau_{1}, h\right), I\left(x, \tau_{1}, h\right)$, а также $L(x, h)$ являются решением задачи (2.25)-(2.27). Тогда при $x \in\left(\tilde{x}_{-}+\varepsilon, \tilde{x}_{+}-\varepsilon\right)$ ВКБ-приближение (2.24) удовлетворяет уравнению (1.11) с точностью $r^{*}$, дде $r^{*}$ имеет вид (2.17).

Так как $g$ должно удовлетворять также условиям (1.12), (1.13), то в силу (2.1), (2.2), (2.19), (2.22), (2.23) имеем

$$
\begin{gathered}
\int_{0}^{\tilde{x}_{-}+\varepsilon} \chi_{-}(x, \varepsilon) \int_{-\infty}^{\infty} g^{2}(x, y) d y d x+\int_{\tilde{x}_{-}+\varepsilon / 2}^{\tilde{x}_{+}-\varepsilon / 2}\left(1-\chi_{-}(x, \varepsilon)-\chi_{+}(x, \varepsilon)\right) \frac{k d x}{2 S^{\prime}(x, h)} \\
+\int_{\tilde{x}_{+}-\varepsilon}^{\infty} \chi_{+}(x, \varepsilon) \int_{-\infty}^{\infty} g^{2}(x, y) d y d x=
\end{gathered}
$$




$$
\begin{aligned}
&=1+ O\left(\int_{\tilde{x}_{-}+\varepsilon / 2}^{\tilde{x}_{+}-\varepsilon / 2}\left(1-\chi_{-}(x, \varepsilon)-\chi_{+}(x, \varepsilon)\right)\right. \\
&\left.\times \int_{-\infty}^{\infty} T^{2}\left(x, \tau_{1}, h\right) \cos 2 \Phi\left(x, \tau_{1}, h\right) d \tau_{1} d x\right) \\
& \int_{0}^{\tilde{x}_{-}+\varepsilon} \chi_{-}(x, \varepsilon) \int_{-\infty}^{\infty} y g^{2}(x, y) d y d x+\frac{h^{5 / 9}}{2} \int_{\tilde{x}_{-}+\varepsilon / 2}^{\tilde{x}_{+}-\varepsilon / 2}\left(1-\chi_{-}(x, \varepsilon)-\chi_{+}(x, \varepsilon)\right) \\
& \times \int_{-\infty}^{\infty} \tau_{1}\left(B^{2}\left(x, \tau_{1}, h\right)+I^{2}\left(x, \tau_{1}, h\right)\right) d \tau_{1} d x \\
&+ \int_{\tilde{x}_{+}-\varepsilon}^{\infty} \chi_{+}(x, \varepsilon) \int_{-\infty}^{\infty} y g^{2}(x, y) d y d x \\
&=O\left(h^{5 / 9} \int_{\tilde{x}_{-}+\varepsilon / 2}^{\tilde{x}_{+}-\varepsilon / 2}\left(1-\chi_{-}(x, \varepsilon)-\chi_{+}(x, \varepsilon)\right)\right. \\
&\left.\quad \times \int_{-\infty}^{\infty} \tau_{1} T^{2}\left(x, \tau_{1}, h\right) \cos 2 \Phi\left(x, \tau_{1}, h\right) d \tau_{1} d x\right)
\end{aligned}
$$

\section{§ 3. Асимптотическое решение задачи для cos- и sin-амплитуд}

В этом параграфе мы построим асимптотическое решение задачи (2.25)-(2.27) в виде

$$
\begin{aligned}
B\left(x, \tau_{1}, h\right) & =B_{0}\left(x, \tau_{1}\right)+h^{2 / 9} B_{1}\left(x, \tau_{1}\right)+h^{4 / 9} B_{2}\left(x, \tau_{1}\right)+O\left(h^{5 / 9} B_{3}\left(x, \tau_{1}\right)\right), \\
I\left(x, \tau_{1}, h\right) & =h^{1 / 9} I_{0}\left(x, \tau_{1}\right)+h^{1 / 3} I_{1}\left(x, \tau_{1}\right)+O\left(h^{5 / 9} I_{2}\left(x, \tau_{1}\right)\right), \\
L(x, h) & =\ell_{0}(x)+h^{2 / 9} \ell_{1}(x)+h^{4 / 9} \ell_{2}(x)+O\left(h^{5 / 9} \ell_{3}(x)\right) .
\end{aligned}
$$

Здесь $x \in\left(\tilde{x}_{-}+\varepsilon, \tilde{x}_{+}-\varepsilon\right), \tau_{1}^{2} \ll h^{-1 / 9}\left|S^{\prime}(x, h)\right|, h \rightarrow 0$. Функции $B_{j}, j=0,1,2,3$, $I_{j}, \quad j=0,1,2, \quad \ell_{j}, \quad j=0,1,2,3$, в (3.1)-(3.3) вешественные, гладкие, причем $B_{j}\left(x, \tau_{1}\right)$ и $I_{j}\left(x, \tau_{1}\right)$ экспоненциально убывают при $\left|\tau_{1}\right| \rightarrow \infty$, а

$$
\ell_{3}(x)=O\left(\left(x-\tilde{x}_{-}\right)^{-7 / 6}\right)+O\left(\left(\tilde{x}_{+}-x\right)^{-7 / 6}\right) .
$$

При этом мы ограничимся лишш формулировкой результатов (более подробно см. [1]).

3.1. Формулы для главного приближения $B_{0}, I_{0}, \ell_{0}$. Подставим разложения для $B, I, L$ в $(2.25),(2.27)$. Тогда для главного члена асимптотики cos-амплитуды имеем

$$
\begin{gathered}
\frac{\partial^{2} B_{0}\left(x, \tau_{1}\right)}{\partial \tau_{1}^{2}}-\left(\frac{1}{2 x} \int_{-\infty}^{\infty}\left|\tau_{1}-\tau_{1}^{\prime}\right| B_{0}^{2}\left(x, \tau_{1}^{\prime}\right) d \tau_{1}^{\prime}+\ell_{0}\right) B_{0}\left(x, \tau_{1}\right)=0, \\
\int_{-\infty}^{\infty} B_{0}^{2}\left(x, \tau_{1}\right) d \tau_{1}=\frac{k}{S^{\prime}}
\end{gathered}
$$


Наряду с (3.4), (3.5) рассмотрим задачу

$$
\begin{gathered}
\frac{d^{2} t(\tau)}{d \tau^{2}}-\left(\pi \int_{-\infty}^{\infty}\left|\tau-\tau^{\prime}\right| t^{2}\left(\tau^{\prime}\right) d \tau^{\prime}+\rho\right) t(\tau)=0, \\
\int_{-\infty}^{\infty} t^{2}(\tau) d \tau=1, \quad \int_{-\infty}^{\infty} \tau t^{2}(\tau) d \tau=0
\end{gathered}
$$

(одномерная модель полярона), которая не зависит от дополнительных параметров. Решение именно этой задачи определяет поведение амплитуды ВКБ-разложения.

Пусть $t=t_{i}(\tau), \rho=\rho_{i}<0, i=1,2, \ldots,-$ собственные функции и собственные значения (3.6), (3.7). (Индекс $i$ для краткости формул мы будем ниже опускать.) Решение (3.4), (3.5) ищем в виде

$$
B_{0}\left(x, \tau_{1}\right)=y(x) t(\tau), \quad \text { где } \tau=\beta(x) \tau_{1}+\varkappa(x) .
$$

ЛЕмма 3.1. Если

$$
y=\frac{1}{(2 \pi x)^{1 / 6}}\left(\frac{k}{S^{\prime}}\right)^{2 / 3}, \quad \beta=\left(\frac{k}{2 \pi x S^{\prime}}\right)^{1 / 3},
$$

то заданная формулой (3.8) функция $B_{0}$, а также

$$
\ell_{0}=\rho\left(\frac{k}{2 \pi x S^{\prime}}\right)^{2 / 3}
$$

являются решением задачи (3.4), (3.5).

ДокАЗАТЕЛЬСтво. Сравнивая (3.4), (3.5) и (3.6), (3.7), имеем

$$
\frac{y^{2}}{2 x \beta^{4}}=\pi, \quad \frac{\ell_{0}}{\beta^{2}}=\rho, \quad \frac{y^{2}}{\beta}=\frac{k}{S^{\prime}},
$$

откуда следуют формулы (3.9), (3.10). Для определенности мы считали, что $k>0$, $S^{\prime}>0, y>0$. Лемма доказана.

Положим $\varkappa(x) \equiv 0$. Тогда

$$
\int_{-\infty}^{\infty} \tau_{1} B_{0}^{2}\left(x, \tau_{1}\right) d \tau_{1}=0
$$

что позволяет обратить в нуль слагаемое порядка $h^{5 / 9}$ в (2.29). Кроме того, такой выбор $\varkappa(x)$ обеспечивает разрешимость задачи для следующего приближения. Таким образом, функция $B_{0}\left(x, \tau_{1}\right)$ полностью определена.

Подставим теперь разложения для $B, I, L$ в $(2.26)$. Тогда для главного члена асимптотики sin-амплитуды получаем уравнение

$$
\widehat{R} I_{0}=2 S^{\prime} \frac{\partial B_{0}}{\partial x}+S^{\prime \prime} B_{0}
$$

где линейный оператор $\widehat{R}$ имеет вид

$$
\widehat{R} I_{0} \stackrel{\text { def }}{=} \frac{\partial^{2} I_{0}}{\partial \tau_{1}^{2}}-\left(\frac{1}{2 x} \int_{-\infty}^{\infty}\left|\tau_{1}-\tau_{1}^{\prime}\right| B_{0}^{2} d \tau_{1}^{\prime}+\ell_{0}\right) I_{0} .
$$


ЗАмЕчаниЕ 3.1. Для упрошения обозначений в (3.14), а также в последуюших формулах этого параграфа, мы не будем выписывать аргументы у функций $B_{j}, j=0,1,2, \varphi_{0}, I_{0}, I_{1}, T_{1}, T_{1,0}$ и их производных. В указанных формулах первый аргумент данных функций равен $x$, а второй либо равен $\tau_{1}$, если функция не стоит под знаком интеграла, либо, в противном случае, совпадает с переменной, по которой производится интегрирование.

Определим

$$
\varphi_{0}\left(x, \tau_{1}\right)=\left(S^{\prime \prime}+\frac{S^{\prime}}{x}\right) \frac{\tau_{1}^{2}}{6} .
$$

Аналогично [1] доказывается

ЛЕмма 3.2. Функиия $I_{0}=-B_{0} \varphi_{0}$ удовлетворяет уравнению (3.13).

3.2. Формулы для первых поправок $B_{1}, \ell_{1}$. Приравнивая нулю в $(2.25)$, $(2.27)$ слагаемые порядка $h^{2 / 9}$, для нахождения $B_{1}, \ell_{1}$ получаем задачу

$$
\begin{gathered}
\widehat{N} B_{1}=\frac{1}{2 x}\left(\int_{-\infty}^{\infty}\left|\tau_{1}-\tau_{1}^{\prime}\right| I_{0}^{2} d \tau_{1}^{\prime}\right) B_{0}+\ell_{1} B_{0}-2 S^{\prime} \frac{\partial I_{0}}{\partial x}-S^{\prime \prime} I_{0}, \\
\int_{-\infty}^{\infty}\left(I_{0}^{2}+2 B_{0} B_{1}\right) d \tau_{1}=0 .
\end{gathered}
$$

Здесь оператор $\widehat{N}$ задан формулой

$$
\begin{aligned}
\widehat{N} B_{1} \stackrel{\text { def }}{=} & \frac{\partial^{2} B_{1}}{\partial \tau_{1}^{2}}-\frac{1}{2 x}\left(\int_{-\infty}^{\infty}\left|\tau_{1}-\tau_{1}^{\prime}\right| B_{0}^{2} d \tau_{1}^{\prime}\right) B_{1} \\
& -\frac{1}{x}\left(\int_{-\infty}^{\infty}\left|\tau_{1}-\tau_{1}^{\prime}\right| B_{0} B_{1} d \tau_{1}^{\prime}\right) B_{0}-\ell_{0} B_{1} .
\end{aligned}
$$

Вместо $B_{1}$ мы будем искать функцию $T_{1}=B_{1}+I_{0}^{2} /\left(2 B_{0}\right)=B_{1}+\varphi_{0}^{2} B_{0} / 2$. Из (3.16), (3.17) вытекает [1]

ЛЕмма 3.3. Функции $T_{1}\left(x, \tau_{1}\right), \ell_{1}(x)$ удовлетворяют задаче

$$
\begin{gathered}
\widehat{N} T_{1}=\left[\ell_{1}+\left(\frac{\partial \varphi_{0}}{\partial \tau_{1}}\right)^{2}+2 S^{\prime} \frac{\partial \varphi_{0}}{\partial x}\right] B_{0}, \\
\int_{-\infty}^{\infty} B_{0} T_{1} d \tau_{1}=0 .
\end{gathered}
$$

Перейдем к изучению задачи (3.19), (3.20). Дифференцируя по $\tau_{1}$ уравнение (3.4), находим, что ядро оператора $\widehat{N}$ содержит гладкую, экспоненциально убываюшую при $\left|\tau_{1}\right| \rightarrow \infty$ функцию $\partial B_{0} / \partial \tau_{1}$. Предположим, что выбрано такое решение задачи (3.6), (3.7), при котором ядро оператора $\widehat{N}$ одномерно. Тогда условием разрешимости уравнения (3.19) будет условие ортогональности в пространстве $L^{2}\left(\mathbb{R}^{1}\right)$ функции $\partial B_{0} / \partial \tau_{1}$ правой части $(3.19)$ :

$$
\int_{-\infty}^{\infty}\left[\ell_{1}+\left(\frac{\partial \varphi_{0}}{\partial \tau_{1}}\right)^{2}+2 S^{\prime} \frac{\partial \varphi_{0}}{\partial x}\right] B_{0} \frac{\partial B_{0}}{\partial \tau_{1}} d \tau_{1}=0
$$


В силу (3.12), (3.15) равенство (3.21) выполнено. Поэтому уравнение (3.19) разрешимо.

Для определения $\ell_{1}(x)$ воспользуемся условием (3.20). Пусть

$$
\Psi\left(x, \tau_{1}\right)=B_{0}+\frac{\tau_{1}}{2} \frac{\partial B_{0}}{\partial \tau_{1}} .
$$

Тогда, используя равенство [1]

$$
\widehat{N}\left(\Psi / \ell_{0}\right)=B_{0}
$$

находим

$$
\ell_{1}(x)=-\frac{\sigma}{27}\left(\frac{2 \pi x S^{\prime}}{k}\right)^{2 / 3}\left\{\left(S^{\prime \prime}+\frac{S^{\prime}}{x}\right)^{2}+3 S^{\prime}\left(S^{\prime \prime}+\frac{S^{\prime}}{x}\right)^{\prime}\right\}
$$

где константы

$$
\sigma=\sigma_{i} \stackrel{\text { def }}{=} \int_{-\infty}^{\infty} \tau^{2} t_{i}^{2}(\tau) d \tau>0, \quad i=1,2, \ldots
$$

Дополним (3.19), (3.20) условием

$$
\int_{-\infty}^{\infty} \tau_{1} B_{0} T_{1} d \tau_{1}=0
$$

что позволяет обратить в нуль слагаемое порядка $h^{7 / 9}$ в (2.29). Кроме того, условие (3.25) обеспечивает разрешимость задачи для следующего приближения (см. [1]).

Задача (3.19), (3.20), (3.25) однозначно определяет $T_{1}$ и $\ell_{1}$. Действительно, если $T_{1,0}\left(x, \tau_{1}\right)$ - некоторое частное решение $(3.19)$, то в силу одномерности ядра $\widehat{N}$ обшее решение (3.19) имеет вид

$$
T_{1}=T_{1,0}+\varkappa_{1}(x) \frac{\partial B_{0}}{\partial \tau_{1}}
$$

где

$$
\varkappa_{1}(x)=\frac{2 S^{\prime}}{k} \int_{-\infty}^{\infty} \tau_{1} B_{0} T_{1,0} d \tau_{1}
$$

находится из условия (3.25).

ЛЕмма 3.4. Справедливо равенство $T_{1}\left(x, \tau_{1}\right)=y_{1}(x) p(\tau)$, әде

$$
y_{1}(x)=\frac{(2 \pi x)^{7 / 6}}{9}\left(\frac{S^{\prime}}{k}\right)^{2 / 3}\left[\left(S^{\prime \prime}+\frac{S^{\prime}}{x}\right)^{2}+3 S^{\prime}\left(S^{\prime \prime}+\frac{S^{\prime}}{x}\right)^{\prime}\right], \quad \tau=\beta(x) \tau_{1},
$$

a $p=p(\tau)$ является решением задачи

$$
\hat{n} p=\left(\tau^{2}-\frac{\sigma}{3}\right) t(\tau), \quad \int_{-\infty}^{\infty} \tau t(\tau) p(\tau) d \tau=0 .
$$

Здесь

$$
\begin{aligned}
\hat{n} p \stackrel{\text { def }}{=} & \frac{d^{2} p}{d \tau^{2}}(\tau)-\pi \int_{-\infty}^{\infty}\left|\tau-\tau^{\prime}\right| t^{2}\left(\tau^{\prime}\right) d \tau^{\prime} p(\tau) \\
& -2 \pi \int_{-\infty}^{\infty}\left|\tau-\tau^{\prime}\right| t\left(\tau^{\prime}\right) p\left(\tau^{\prime}\right) d \tau^{\prime} t(\tau)-\rho p(\tau) .
\end{aligned}
$$


3.3. Формулы для поправок $B_{2}, I_{1}, \ell_{2}$. Для нахождения функций $B_{2}, I_{1}$ и $\ell_{2}$ из (2.25)-(2.27) получаем задачу

$$
\begin{aligned}
\widehat{R} I_{1}= & \left(\frac{1}{2 x} \int_{-\infty}^{\infty}\left|\tau_{1}-\tau_{1}^{\prime}\right|\left(I_{0}^{2}+2 B_{0} B_{1}\right) d \tau_{1}^{\prime}+\ell_{1}\right) I_{0}+2 S^{\prime} \frac{\partial B_{1}}{\partial x}+S^{\prime \prime} B_{1} \\
\widehat{N} B_{2}= & \left(\frac{1}{2 x} \int_{-\infty}^{\infty}\left|\tau_{1}-\tau_{1}^{\prime}\right|\left(I_{0}^{2}+2 B_{0} B_{1}\right) d \tau_{1}^{\prime}+\ell_{1}\right) B_{1} \\
& +\left(\frac{1}{2 x} \int_{-\infty}^{\infty}\left|\tau_{1}-\tau_{1}^{\prime}\right|\left(B_{1}^{2}+2 I_{0} I_{1}\right) d \tau_{1}^{\prime}+\ell_{2}\right) B_{0}-2 S^{\prime} \frac{\partial I_{1}}{\partial x}-S^{\prime \prime} I_{1} \\
& \int_{-\infty}^{\infty}\left(B_{1}^{2}+2 B_{0} B_{2}+2 I_{0} I_{1}\right) d \tau_{1}=0
\end{aligned}
$$

Здесь операторы $\widehat{R}$ и $\widehat{N}$ заданы формулами (3.14), (3.18), а аргументы у функций опушены в соответствии с замечанием 3.1 .

Потребуем также, чтобы

$$
\int_{-\infty}^{\infty} \tau_{1}\left(B_{1}^{2}+2 B_{0} B_{2}+2 I_{0} I_{1}\right) d \tau_{1}=0
$$

Тогда уравнения (3.27)-(3.30) однозначно определяют как $I_{1}$, так и $B_{2}$. Справедлива

ЛЕМма 3.5. Функиия $I_{1}$ имеет вид

$$
I_{1}=y(x) \psi(x) z(\tau)-\varphi_{0} T_{1}+\varphi_{0}^{3} B_{0} / 6,
$$

əде

$$
\begin{aligned}
\psi(x)= & \frac{2}{9}\left(\frac{2 \pi x S^{\prime}}{k}\right)^{2}\left\{\frac{1}{3}\left[\left(S^{\prime \prime}+\frac{S^{\prime}}{x}\right)^{3}+3 S^{\prime}\left(S^{\prime \prime}+\frac{S^{\prime}}{x}\right)\left(S^{\prime \prime}+\frac{S^{\prime}}{x}\right)^{\prime}\right]\right. \\
& \left.+\frac{1}{x}\left[x\left(S^{\prime}\left(S^{\prime \prime}+\frac{S^{\prime}}{x}\right)^{2}+3\left(S^{\prime}\right)^{2}\left(S^{\prime \prime}+\frac{S^{\prime}}{x}\right)\right)\right]^{\prime}\right\}
\end{aligned}
$$

a $z=z(\tau)$ является решением задачи

$$
\begin{gathered}
\frac{d^{2} z(\tau)}{d \tau^{2}}-\left(\pi \int_{-\infty}^{\infty}\left|\tau-\tau^{\prime}\right| t^{2}\left(\tau^{\prime}\right) d \tau^{\prime}+\rho\right) z(\tau)=p(\tau) \\
\int_{-\infty}^{\infty}\left(\frac{\tau}{2} \frac{d t}{d \tau}(\tau)+t(\tau)\right) z(\tau) d \tau=0
\end{gathered}
$$

Для нахождения $\ell_{2}$ воспользуемся условием (3.29). В силу (3.22) получаем

$$
\ell_{2}(x)=-\frac{4}{3}\left(\frac{S^{\prime}}{k}\right)^{2 / 3} \frac{y_{1}^{2} u}{(2 \pi x)^{1 / 3}} .
$$

Здесь константы имеют вид

$$
\begin{aligned}
u= & u_{i} \stackrel{\text { def }}{=} \int_{-\infty}^{\infty}\left(\frac{\tau}{2} \frac{d t_{i}(\tau)}{d \tau}+t_{i}(\tau)\right)\left\{-\frac{\sigma_{i}}{3} p(\tau)+2 \pi \int_{-\infty}^{\infty}\left|\tau-\tau^{\prime}\right| t_{i}\left(\tau^{\prime}\right) p\left(\tau^{\prime}\right) d \tau^{\prime} p(\tau)\right. \\
& \left.+\pi \int_{-\infty}^{\infty}\left|\tau-\tau^{\prime}\right| p^{2}\left(\tau^{\prime}\right) d \tau^{\prime} t_{i}(\tau)+\tau^{2} p(\tau)\right\} d \tau+\frac{\rho_{i}}{2} \int_{-\infty}^{\infty} p^{2}(\tau) d \tau
\end{aligned}
$$

при $i=1,2, \ldots$.

Наконец, справедлива 
ЛЕмма 3.6. Функция $B_{2}$ представима в виде $B_{2}=T_{2}-\varphi_{0}^{2} T_{1} / 2+\varphi_{0} y \psi z(\tau)+$ $\varphi_{0}^{4} B_{0} / 4$ !, где $T_{2}\left(x, \tau_{1}\right)=y_{1}^{2} t_{2,1}(\tau) / y+2 S^{\prime} \psi^{\prime} \sqrt{2 \pi x} t_{2,2}(\tau)$. Здесь $\tau=\beta(x) \tau_{1}, a$ $t_{2,1}(\tau), t_{2,2}(\tau)-$ решения следующих задач:

$$
\begin{gathered}
\hat{n} t_{2,1}=-\frac{\sigma}{3} p(\tau)-\frac{4}{3} u t(\tau)+2 \pi \int_{-\infty}^{\infty}\left|\tau-\tau^{\prime}\right| t\left(\tau^{\prime}\right) p\left(\tau^{\prime}\right) d \tau^{\prime} p(\tau) \\
+\pi \int_{-\infty}^{\infty}\left|\tau-\tau^{\prime}\right| p^{2}\left(\tau^{\prime}\right) d \tau^{\prime} t(\tau)+\tau^{2} p(\tau) \\
\int_{-\infty}^{\infty} \tau\left(2 t_{2,1}(\tau) t(\tau)+p^{2}(\tau)\right) d \tau=0 \\
\hat{n} t_{2,2}=-z(\tau), \quad \int_{-\infty}^{\infty} \tau t_{2,2}(\tau) t(\tau) d \tau=0
\end{gathered}
$$

Oператор $\hat{n}$ в (3.33) задан формулой (3.26).

\section{$\S 4$. Асимптотика в окрестности точек поворота. Модельное уравнение}

Построим асимптотическое решение уравнения (1.11) в окрестности левой точки поворота $\tilde{x}_{-}$, а точнее, на интервале $\left|x-\tilde{x}_{-}\right|<2 \varepsilon$. Здесь $\varepsilon=c h^{26 / 57}$, где $c>0-$ произвольная константа, а точка $\tilde{x}_{-}$удовлетворяет уравнению (2.4). Решение будем искать в виде

$$
\begin{aligned}
g= & g_{-}(x, y)+O\left(h^{1 / 6} \xi^{2} \frac{\partial G_{-}}{\partial \xi}\right)+O\left(h^{-\frac{1}{6}+\frac{1}{57}} \ln \frac{1}{h} \frac{G_{-}}{(1+|\xi|)}\right) \\
& +O\left(h^{-\frac{1}{6}+\frac{1}{57}} \ln \frac{1}{h} \frac{\partial G_{-}}{\partial \xi}\right)+O\left(h^{-\frac{1}{18}+\frac{20}{171}} \ln \frac{1}{h} \xi \frac{\partial G_{-}}{\partial \xi}\right)
\end{aligned}
$$

где

$$
\begin{gathered}
g_{-}(x, y)=\Omega_{-}^{2 / 3} \sqrt{2 \pi \tilde{x}_{-}} G_{-}(\xi, \eta) / \sqrt{h}, \\
\xi=\left(x-\tilde{x}_{-}\right) \sqrt[3]{\Omega_{-}} / h^{2 / 3}, \quad \eta=y \sqrt[3]{\Omega_{-}} / h^{2 / 3} .
\end{gathered}
$$

Функция $G_{-}(\xi, \eta)$, а также константа $\Omega_{-}$определяются в процессе построения асимптотики. Для упрощения обозначений мы не будем явно указывать зависимость $g_{-}$от параметра $h$.

Отметим, что основная сложность при выводе модельного уравнения для $G_{-}$ связана с нелокальностью задачи, которая приводит к тому, что на асимптотику решения $g$ вблизи точки $\tilde{x}_{-}$влияет поведение решения вдали от точки $\tilde{x}_{-}$.

Разложим ядро интегрального оператора в $(2.10)$ при $x \rightarrow \tilde{x}_{-}$. Для этого нам необходима более точная, чем $(2.6)$, асимптотика $K(\mathbb{k})$ при $\mathbb{k} \rightarrow 1$.

ЛЕмма 4.1. Пусть $b=\mathbb{k}^{2}$. Тогда справедливо равенство

$$
\frac{d K(\mathbb{k})}{d b}=\frac{1}{2(1-b)}+\frac{1}{8} \ln (1-b)-\frac{\ln 2}{2}+\frac{3}{8}+O((1-b) \ln (1-b)), \quad b \rightarrow 1 .
$$


ДОКАЗАТЕЛЬСТво. В силу (2.6), разложений [10]

$$
K(\mathbb{k})=(\pi / 2)\left(1+b / 4+O\left(b^{2}\right)\right), \quad E(\mathbb{k})=(\pi / 2)\left(1-b / 4+O\left(b^{2}\right)\right), \quad b \rightarrow 0,
$$

а также соотношения Лежандра [7]

$$
K(\mathbb{k}) E\left(\mathbb{k}^{\prime}\right)+K\left(\mathbb{k}^{\prime}\right) E(\mathbb{k})-K(\mathbb{k}) K\left(\mathbb{k}^{\prime}\right)=\pi / 2,
$$

где $\mathbb{k}^{\prime}=\sqrt{1-\mathbb{k}^{2}}$, получаем

$$
\begin{gathered}
E(\mathbb{k})=1-\frac{1}{4}(1-b) \ln (1-b)+\left(\ln 2-\frac{1}{4}\right)(1-b) \\
+O\left((1-b)^{2} \ln (1-b)\right), \quad b \rightarrow 1
\end{gathered}
$$

Далее, подставляя (4.4), (2.6) в (2.8), находим

$$
\frac{d K(\mathbb{k})}{d b}=\frac{1}{2(1-b)}+\frac{1}{8} \ln (1-b)-\frac{\ln 2}{2}+\frac{3}{8}+O((1-b) \ln (1-b)), \quad b \rightarrow 1 .
$$

Лемма доказана.

Введем функцию

$$
\begin{aligned}
F_{-}\left(x^{\prime}, \tilde{x}_{-}\right) \stackrel{\text { def }}{=} & \frac{2}{\pi}\left[\frac{1}{\left(x^{\prime}-\tilde{x}_{-}\right)}\left(\frac{1}{2 \tilde{x}_{-}} E\left(\frac{2 \sqrt{\tilde{x}_{-} x^{\prime}}}{\tilde{x}_{-}+x^{\prime}}\right)-\frac{1}{\tilde{x}_{-}+x^{\prime}}\right)\right. \\
& \left.-\frac{1}{2 \tilde{x}_{-}\left(\tilde{x}_{-}+x^{\prime}\right)} K\left(\frac{2 \sqrt{\tilde{x}_{-} x^{\prime}}}{\tilde{x}_{-}+x^{\prime}}\right)\right] .
\end{aligned}
$$

Она имеет логарифмическую особенность при $x^{\prime} \rightarrow \tilde{x}_{-}$:

$$
F_{-}\left(x^{\prime}, \tilde{x}_{-}\right)=O\left(|\ln | x^{\prime}-\tilde{x}_{-}||+1\right) \text {. }
$$

Лемма 4.2. Имеет место равенство

$$
\begin{aligned}
& \frac{2}{\pi\left(x+x^{\prime}\right)} K\left(\frac{2 \sqrt{x x^{\prime}}}{x+x^{\prime}}\right)=\frac{2}{\pi\left(\tilde{x}_{-}+x^{\prime}\right)} K\left(\frac{2 \sqrt{\tilde{x}_{-} x^{\prime}}}{\tilde{x}_{-}+x^{\prime}}\right)-\frac{2}{\pi\left(\tilde{x}_{-}+x^{\prime}\right)} \ln \left|\frac{x-x^{\prime}}{\frac{\tilde{x}_{-}-x^{\prime}}{}}\right| \\
& \quad+\left(x-\tilde{x}_{-}\right) F_{-}\left(x^{\prime}, \tilde{x}_{-}\right)+\frac{2}{\pi} \frac{\left(x-\tilde{x}_{-}\right)}{\left(\tilde{x}_{-}+x^{\prime}\right)^{2}} \ln \left|\frac{x-x^{\prime}}{\tilde{x}_{-}-x^{\prime}}\right| \\
& \quad+O\left(\frac{\left(x-\tilde{x}_{-}\right)^{2}}{\left(\tilde{x}_{-}+x^{\prime}\right)^{3}}\left(1+|\ln | x-x^{\prime}||+|\ln | \tilde{x}_{-}-x^{\prime}||\right)\right), \quad x \rightarrow \tilde{x}_{-}
\end{aligned}
$$


ДокАЗАТЕЛЬСТво. Используя (4.3), (2.8), получаем

$$
K\left(\frac{2 \sqrt{x x^{\prime}}}{x+x^{\prime}}\right)-K\left(\frac{2 \sqrt{\tilde{x}_{-} x^{\prime}}}{\tilde{x}_{-}+x^{\prime}}\right)=\int_{4 \tilde{x}_{-} x^{\prime} /\left(\tilde{x}_{-}+x^{\prime}\right)^{2}}^{4 x x^{\prime} /\left(x+x^{\prime}\right)^{2}} \frac{d K}{d b}(\sqrt{b}) d b=\delta K_{1}+\delta K_{2}
$$

Здесь

$$
\begin{aligned}
& \delta K_{1} \stackrel{\text { def }}{=} \int_{4 \tilde{x}_{-} x^{\prime} /\left(\tilde{x}_{-}+x^{\prime}\right)^{2}}^{4 x x^{\prime} /\left(x+x^{\prime}\right)^{2}}\left\{\frac{1}{2(1-b)}+\frac{1}{8} \ln (1-b)\right\} d b \\
& \delta K_{2} \stackrel{\text { def }}{=} \int_{4 \tilde{x}_{-} x^{\prime} /\left(\tilde{x}_{-}+x^{\prime}\right)^{2}}^{4 x x^{\prime} /\left(x+x^{\prime}\right)^{2}}\left\{\frac{1}{2(1-b)}\left[\frac{E(\sqrt{b})}{b}-1\right]-\frac{1}{2 b} K(\sqrt{b})-\frac{1}{8} \ln (1-b)\right\} d b,
\end{aligned}
$$

причем $\delta K_{1}$ содержит члены разложения (4.3), имеюшие особенность при $b \rightarrow 1$.

Вычислим входящий в $\delta K_{1}$ интеграл:

$$
\begin{aligned}
\delta K_{1}= & \left.\left\{-\frac{1}{2} \ln (1-b)-\frac{1}{8}(1-b)[\ln (1-b)-1]\right\}\right|_{4 \tilde{x}_{-} x^{\prime} /\left(\tilde{x}_{-}+x^{\prime}\right)^{2}} ^{4 x x^{\prime} /\left(x+x^{\prime}\right)^{2}} \\
= & -\ln \left|\frac{x-x^{\prime}}{\tilde{x}_{-}-x^{\prime}}\right|+\left(x-\tilde{x}_{-}\right)\left[\frac{1}{\tilde{x}_{-}+x^{\prime}}-\frac{x^{\prime}\left(\tilde{x}_{-}-x^{\prime}\right)}{\left(\tilde{x}_{-}+x^{\prime}\right)^{3}} \ln \left|\frac{\tilde{x}_{-}-x^{\prime}}{\tilde{x}_{-}+x^{\prime}}\right|\right] \\
& +O\left(\frac{\left(x-\tilde{x}_{-}\right)^{2}}{\left(\tilde{x}_{-}+x^{\prime}\right)^{2}}\left(1+|\ln | \tilde{x}_{-}-x^{\prime}||\right)\right), \quad x \rightarrow \tilde{x}_{-} .
\end{aligned}
$$

Далее, разлагая $\delta K_{2}$ по формуле Тейлора, находим

$$
\begin{aligned}
\delta K_{2}= & \frac{\left(x-\tilde{x}_{-}\right) 4 x^{\prime}\left(x^{\prime}-\tilde{x}_{-}\right)}{\left(\tilde{x}_{-}+x^{\prime}\right)^{3}}\left\{\frac{1}{2} \frac{\left(\tilde{x}_{-}+x^{\prime}\right)^{2}}{\left(\tilde{x}_{-}-x^{\prime}\right)^{2}}\left[E\left(\frac{2 \sqrt{\tilde{x}_{-} x^{\prime}}}{\tilde{x}_{-}+x^{\prime}}\right) \frac{\left(\tilde{x}_{-}+x^{\prime}\right)^{2}}{4 \tilde{x}_{-} x^{\prime}}-1\right]\right. \\
& \left.-\frac{\left(\tilde{x}_{-}+x^{\prime}\right)^{2}}{8 \tilde{x}_{-} x^{\prime}} K\left(\frac{2 \sqrt{\tilde{x}_{-} x^{\prime}}}{\tilde{x}_{-}+x^{\prime}}\right)-\frac{1}{4} \ln \left|\frac{\tilde{x}_{-}-x^{\prime}}{\tilde{x}_{-}+x^{\prime}}\right|\right\} \\
& +O\left(\frac{\left(x-\tilde{x}_{-}\right)^{2}}{\left(\tilde{x}_{-}+x^{\prime}\right)^{2}}\left(1+|\ln | \tilde{x}_{-}-x^{\prime}||\right)\right), \quad x \rightarrow \tilde{x}_{-}
\end{aligned}
$$

Таким образом,

$$
\begin{aligned}
& \frac{2}{\pi\left(x+x^{\prime}\right)} K\left(\frac{2 \sqrt{x x^{\prime}}}{x+x^{\prime}}\right)=\frac{2}{\pi}\left(\frac{1}{\tilde{x}_{-}+x^{\prime}}-\frac{x-\tilde{x}_{-}}{\left(\tilde{x}_{-}+x^{\prime}\right)^{2}}+O\left(\frac{\left(x-\tilde{x}_{-}\right)^{2}}{\left(\tilde{x}_{-}+x^{\prime}\right)^{3}}\right)\right) \\
& \quad \times\left\{K\left(\frac{2 \sqrt{\tilde{x}_{-} x^{\prime}}}{\tilde{x}_{-}+x^{\prime}}\right)-\ln \left|\frac{x-x^{\prime}}{\tilde{x}_{-}-x^{\prime}}\right|+\left(x-\tilde{x}_{-}\right)\left[\frac{1}{\tilde{x}_{-}-x^{\prime}}\right.\right. \\
& \left.+\frac{\left(\tilde{x}_{-}+x^{\prime}\right)}{2 \tilde{x}_{-}\left(x^{\prime}-\tilde{x}_{-}\right)} E\left(\frac{2 \sqrt{\tilde{x}_{-} x^{\prime}}}{\tilde{x}_{-}+x^{\prime}}\right)+\frac{\left(\tilde{x}_{-}-x^{\prime}\right)}{2 \tilde{x}_{-}\left(\tilde{x}_{-}+x^{\prime}\right)} K\left(\frac{2 \sqrt{\tilde{x}_{-} x^{\prime}}}{\tilde{x}_{-}+x^{\prime}}\right)\right] \\
& \left.+O\left(\frac{\left(x-\tilde{x}_{-}\right)^{2}}{\left(\tilde{x}_{-}+x^{\prime}\right)^{2}}\left(1+|\ln | \tilde{x}_{-}-x^{\prime}||\right)\right)\right\}, \quad x \rightarrow \tilde{x}_{-},
\end{aligned}
$$


а значит, справедливо разложение (4.7), где $F_{-}$задается соотношением (4.5). Лемма доказана.

Равенства (4.7), (2.4) позволяют записать уравнение (2.10) вблизи точки $\tilde{x}_{-}$в виде

$$
\begin{aligned}
h^{2}( & \left.\frac{\partial^{2} g}{\partial x^{2}}+\frac{\partial^{2} g}{\partial y^{2}}\right) \\
& +\left\{U^{\prime}\left(\tilde{x}_{-}\right)\left(x-\tilde{x}_{-}\right)+h^{1 / 3}\left(x-\tilde{x}_{-}\right) \int_{0}^{\infty} F_{-}\left(x^{\prime}, \tilde{x}_{-}\right) \int_{-\infty}^{\infty} g^{2}\left(x^{\prime}, y^{\prime}\right) d y^{\prime} d x^{\prime}\right. \\
& -h^{1 / 3} \int_{0}^{\infty} \frac{1}{\pi\left(\tilde{x}_{-}+x^{\prime}\right)} \int_{-\infty}^{\infty} \ln \left[\frac{\left(x-x^{\prime}\right)^{2}+\left(y-y^{\prime}\right)^{2}}{\left(\tilde{x}_{-}-x^{\prime}\right)^{2}}\right] g^{2}\left(x^{\prime}, y^{\prime}\right) d y^{\prime} d x^{\prime} \\
& +O\left(h^{1 / 3}\left(x-\tilde{x}_{-}\right) \int_{0}^{\infty} \frac{1}{\left(\tilde{x}_{-}+x^{\prime}\right)^{2}}\right. \\
& \left.\times \int_{-\infty}^{\infty} \ln \left[\frac{\left(x-x^{\prime}\right)^{2}+\left(y-y^{\prime}\right)^{2}}{\left(\tilde{x}_{-}-x^{\prime}\right)^{2}}\right] g^{2}\left(x^{\prime}, y^{\prime}\right) d y^{\prime} d x^{\prime}\right) \\
& +O\left(h^{1 / 3} \int_{0}^{\infty} \int_{-\infty}^{\infty} \frac{\left(y-y^{\prime}\right)^{2}}{\left(\tilde{x}_{-}+x^{\prime}\right)^{3}}\left(1+|\ln | x-x^{\prime}||\right) g^{2}\left(x^{\prime}, y^{\prime}\right) d y^{\prime} d x^{\prime}\right) \\
& \left.+O\left(\left(x-\tilde{x}_{-}\right)^{2}\right)+O\left(h^{1+\frac{1}{57}} \ln \frac{1}{h}\right)+O\left(y^{2}\right)\right\} g=0
\end{aligned}
$$

Разлагая затем по формуле Тейлора входящий в (4.8) интеграл, имеем

$$
\begin{aligned}
\int_{0}^{\infty} & \frac{1}{\pi\left(\tilde{x}_{-}+x^{\prime}\right)} \int_{-\infty}^{\infty} \ln \left[\frac{\left(x-x^{\prime}\right)^{2}+\left(y-y^{\prime}\right)^{2}}{\left(\tilde{x}_{-}-x^{\prime}\right)^{2}}\right] g^{2}\left(x^{\prime}, y^{\prime}\right) d y^{\prime} d x^{\prime} g(x, y) \\
= & \left\{\frac{1}{2 \pi \tilde{x}_{-}} \iint_{-\infty}^{\infty} \ln \left[\frac{\left(x-x^{\prime}\right)^{2}+\left(y-y^{\prime}\right)^{2}}{\left(\tilde{x}_{-}-x^{\prime}\right)^{2}}\right] g_{-}^{2}\left(x^{\prime}, y^{\prime}\right) d y^{\prime} d x^{\prime}\right. \\
& +\frac{1}{\pi} \iint_{-\infty}^{\infty} \ln \left[\frac{\left(x-x^{\prime}\right)^{2}+\left(y-y^{\prime}\right)^{2}}{\left(\tilde{x}_{-}-x^{\prime}\right)^{2}}\right] \\
& \left.\times\left(\frac{\theta\left(x^{\prime}\right)}{\tilde{x}_{-}+x^{\prime}} g^{2}\left(x^{\prime}, y^{\prime}\right)-\frac{g_{-}^{2}\left(x^{\prime}, y^{\prime}\right)}{2 \tilde{x}_{-}}\right) d y^{\prime} d x^{\prime}\right\} g(x, y) \\
= & \left\{\frac{1}{2 \pi \tilde{x}_{-}} \iint_{-\infty}^{\infty} \ln \left[\frac{\left(x-x^{\prime}\right)^{2}+\left(y-y^{\prime}\right)^{2}}{\left(\tilde{x}_{-}-x^{\prime}\right)^{2}}\right] g_{-}^{2}\left(x^{\prime}, y^{\prime}\right) d y^{\prime} d x^{\prime}\right. \\
& -\frac{2\left(x-\tilde{x}_{-}\right)}{\pi} \int_{\tilde{x}_{-}+\varepsilon}^{\infty} \frac{\left(1-\chi_{-}^{*}\left(x^{\prime}, \varepsilon\right)\right)}{\left(x^{\prime}-\tilde{x}_{-}\right)} \int_{-\infty}^{\infty}\left[\frac{g^{2}\left(x^{\prime}, y^{\prime}\right)}{\tilde{x}_{-}+x^{\prime}}-\frac{g_{-}^{2}\left(x^{\prime}, y^{\prime}\right)}{2 \tilde{x}_{-}}\right] d y^{\prime} d x^{\prime} \\
& +O\left(\left(x-\tilde{x}_{-}\right)^{2} \int_{\tilde{x}_{-}+\varepsilon}^{\infty} \frac{\left(1-\chi_{-}^{*}\left(x^{\prime}, \varepsilon\right)\right)}{\left(x^{\prime}-\tilde{x}_{-}\right)^{2}} \int_{-\infty}^{\infty}\left[\frac{g^{2}\left(x^{\prime}, y^{\prime}\right)}{\tilde{x}_{-}+x^{\prime}}-\frac{g_{-}^{2}\left(x^{\prime}, y^{\prime}\right)}{2 \tilde{x}_{-}}\right] d y^{\prime} d x^{\prime}\right) \\
& +O\left(\int_{\tilde{x}_{-}+\varepsilon}^{\infty}\left(1-\chi_{-}^{*}\left(x^{\prime}, \varepsilon\right)\right)\right. \\
& \times \int_{-\infty}^{\infty} \ln \left[1+\frac{\left(y-y^{\prime}\right)^{2}}{\left(x-x^{\prime}\right)^{2}}\right]\left[\frac{g^{2}\left(x^{\prime}, y^{\prime}\right)}{\tilde{x}_{-}+x^{\prime}}-\frac{g_{-}^{2}\left(x^{\prime}, y^{\prime}\right)}{\left.\left.2 \tilde{x}_{-}\right] d y^{\prime} d x^{\prime}\right)}\right.
\end{aligned}
$$




$$
\begin{aligned}
+O & \left(\int_{-\infty}^{\tilde{x}_{-}+2 \varepsilon} \chi_{-}^{*}\left(x^{\prime}, \varepsilon\right) \int_{-\infty}^{\infty} \ln \left[\frac{\left(x-x^{\prime}\right)^{2}+\left(y-y^{\prime}\right)^{2}}{\left(\tilde{x}_{-}-x^{\prime}\right)^{2}}\right]\right. \\
& \left.\left.\times\left(\frac{\theta\left(x^{\prime}\right)}{\tilde{x}_{-}+x^{\prime}} g^{2}\left(x^{\prime}, y^{\prime}\right)-\frac{g_{-}^{2}\left(x^{\prime}, y^{\prime}\right)}{2 \tilde{x}_{-}}\right) d y^{\prime} d x^{\prime}\right)\right\} g(x, y) .
\end{aligned}
$$

Здесь $\chi_{-}^{*}(x, \varepsilon) \stackrel{\text { def }}{=} \chi\left(\left(x-\tilde{x}_{-}\right) /(2 \varepsilon)\right)$,

$$
\theta(x)=\left\{\begin{array}{lll}
1 & \text { при } & x>0 \\
0 & \text { при } & x \leqslant 0
\end{array}\right.
$$

Будем считать решение $g$ при $x>\tilde{x}_{-}+\varepsilon$ известным. Далее подставим в (4.8), (4.9) при $\left|x-\tilde{x}_{-}\right|<2 \varepsilon$ вместо $g$ функцию $g_{-}$. Тогда для нахождения $g_{-}$получаем уравнение

$$
\begin{aligned}
h^{2}\left(\frac{\partial^{2} g_{-}}{\partial x^{2}}+\frac{\partial^{2} g_{-}}{\partial y^{2}}\right)+\left\{\Omega_{-}\left(x-\tilde{x}_{-}\right)\right. \\
\left.\quad-\frac{h^{1 / 3}}{2 \pi \tilde{x}_{-}} \iint_{-\infty}^{\infty} \ln \left[\frac{\left(x-x^{\prime}\right)^{2}+\left(y-y^{\prime}\right)^{2}}{\left(x^{\prime}-\tilde{x}_{-}\right)^{2}}\right] g_{-}^{2}\left(x^{\prime}, y^{\prime}\right) d y^{\prime} d x^{\prime}\right\} g_{-}=0
\end{aligned}
$$

где

$$
\begin{aligned}
\Omega_{-}= & U^{\prime}\left(\tilde{x}_{-}\right)+\frac{2 h^{1 / 3}}{\pi} \int_{\tilde{x}_{-}+\varepsilon}^{\infty} \frac{\left(1-\chi_{-}^{*}\left(x^{\prime}, \varepsilon\right)\right)}{\left(x^{\prime}-\tilde{x}_{-}\right)} \int_{-\infty}^{\infty}\left(\frac{g^{2}\left(x^{\prime}, y^{\prime}\right)}{\tilde{x}_{-}+x^{\prime}}-\frac{g_{-}^{2}\left(x^{\prime}, y^{\prime}\right)}{2 \tilde{x}_{-}}\right) d y^{\prime} d x^{\prime} \\
& +h^{1 / 3} \int_{0}^{\infty} F_{-}\left(x^{\prime}, \tilde{x}_{-}\right) \times \\
& \times \int_{-\infty}^{\infty}\left(\chi_{-}^{*}\left(x^{\prime}, \varepsilon\right) g_{-}^{2}\left(x^{\prime}, y^{\prime}\right)+\left(1-\chi_{-}^{*}\left(x^{\prime}, \varepsilon\right)\right) g^{2}\left(x^{\prime}, y^{\prime}\right)\right) d y^{\prime} d x^{\prime}= \\
= & U^{\prime}\left(\tilde{x}_{-}\right)+\frac{h^{1 / 3}}{\pi \tilde{x}_{-}} \int_{\tilde{x}_{-}+\varepsilon}^{\infty}\left(1-\chi_{-}^{*}\left(x^{\prime}, \varepsilon\right)\right) \int_{-\infty}^{\infty}\left\{\left[\frac{1}{\left(x^{\prime}-\tilde{x}_{-}\right)} E\left(\frac{2 \sqrt{\tilde{x}_{-} x^{\prime}}}{\tilde{x}_{-}+x^{\prime}}\right)\right.\right. \\
& \left.\left.-\frac{1}{\left(\tilde{x}_{-}+x^{\prime}\right)} K\left(\frac{2 \sqrt{\tilde{x}_{-} x^{\prime}}}{\tilde{x}_{-}+x^{\prime}}\right)\right] g^{2}\left(x^{\prime}, y^{\prime}\right)-\frac{g_{-}^{2}\left(x^{\prime}, y^{\prime}\right)}{x^{\prime}-\tilde{x}_{-}}\right\} d y^{\prime} d x^{\prime} \\
& +O\left(h^{\frac{4}{9}+\frac{20}{171}} \ln \frac{1}{h}\right) .
\end{aligned}
$$

При этом в уравнении (4.8) возникает невязка

$$
\begin{aligned}
r_{-}= & \left\{O\left(\left(x-\tilde{x}_{-}\right)^{2}\right)+O\left(h^{1+\frac{1}{57}} \ln \frac{1}{h}\right)+O\left(y^{2}\right)+O\left(h^{\frac{4}{9}+\frac{20}{171}} \ln \frac{1}{h}\left(x-\tilde{x}_{-}\right)\right)\right. \\
& +O\left(h^{1 / 3}\left(x-\tilde{x}_{-}\right)^{2} \int_{\tilde{x}_{-}+\varepsilon}^{\infty} \frac{\left(1-\chi_{-}^{*}\left(x^{\prime}, \varepsilon\right)\right)}{\left(x^{\prime}-\tilde{x}_{-}\right)^{2}}\right. \\
& \left.\times \int_{-\infty}^{\infty}\left(\frac{g^{2}\left(x^{\prime}, y^{\prime}\right)}{\tilde{x}_{-}+x^{\prime}}-\frac{g_{-}^{2}\left(x^{\prime}, y^{\prime}\right)}{2 \tilde{x}_{-}}\right) d y^{\prime} d x^{\prime}\right)
\end{aligned}
$$




$$
\begin{aligned}
& +O\left(h^{1 / 3} \int_{\tilde{x}_{-}+\varepsilon}^{\infty}\left(1-\chi_{-}^{*}\left(x^{\prime}, \varepsilon\right)\right) \int_{-\infty}^{\infty} \ln \left[1+\frac{\left(y-y^{\prime}\right)^{2}}{\left(x-x^{\prime}\right)^{2}}\right]\right. \\
& \left.\times\left(\frac{g^{2}\left(x^{\prime}, y^{\prime}\right)}{\tilde{x}_{-}+x^{\prime}}-\frac{g_{-}^{2}\left(x^{\prime}, y^{\prime}\right)}{2 \tilde{x}_{-}}\right) d y^{\prime} d x^{\prime}\right) \\
& +O\left(h^{1 / 3} \int_{-\infty}^{\tilde{x}_{-}+2 \varepsilon} \chi_{-}^{*}\left(x^{\prime}, \varepsilon\right) \int_{-\infty}^{\infty} \ln \left(\frac{\left(x-x^{\prime}\right)^{2}+\left(y-y^{\prime}\right)^{2}}{\left(\tilde{x}_{-}-x^{\prime}\right)^{2}}\right)\right. \\
& \left.\times\left(\frac{\theta\left(x^{\prime}\right)}{\tilde{x}_{-}+x^{\prime}}-\frac{1}{2 \tilde{x}_{-}}\right) g_{-}^{2}\left(x^{\prime}, y^{\prime}\right) d y^{\prime} d x^{\prime}\right) \\
& +O\left(h^{1 / 3}\left(x-\tilde{x}_{-}\right) \int_{0}^{\infty} \frac{1}{\left(\tilde{x}_{-}+x^{\prime}\right)^{2}} \int_{-\infty}^{\infty} \ln \left(\frac{\left(x-x^{\prime}\right)^{2}+\left(y-y^{\prime}\right)^{2}}{\left(\tilde{x}_{-}-x^{\prime}\right)^{2}}\right)\right. \\
& \left.\times\left[\chi_{-}^{*}\left(x^{\prime}, \varepsilon\right) g_{-}^{2}\left(x^{\prime}, y^{\prime}\right)+\left(1-\chi_{-}^{*}\left(x^{\prime}, \varepsilon\right)\right) g^{2}\left(x^{\prime}, y^{\prime}\right)\right] d y^{\prime} d x^{\prime}\right) \\
& +O\left(h^{1 / 3} \int_{0}^{\infty} \int_{-\infty}^{\infty} \frac{\left(y-y^{\prime}\right)^{2}}{\left(\tilde{x}_{-}+x^{\prime}\right)^{3}}\left(1+|\ln | x-x^{\prime}||\right)\right. \\
& \left.\left.\times\left[\chi_{-}^{*}\left(x^{\prime}, \varepsilon\right) g_{-}^{2}\left(x^{\prime}, y^{\prime}\right)+\left(1-\chi_{-}^{*}\left(x^{\prime}, \varepsilon\right)\right) g^{2}\left(x^{\prime}, y^{\prime}\right)\right] d y^{\prime} d x^{\prime}\right)\right\} g_{-}(x, y) .
\end{aligned}
$$

Наконец, сделав в (4.11) замену (4.1), (4.2), получаем следующую лемму.

Лемма 4.3. Функиия $G_{-}(\xi, \eta)$ удовлетворяет модельному уравнению (1.4). Аналогично, при $\left|x-\tilde{x}_{+}\right|<2 \varepsilon$ асимптотику ишем в виде

$$
\begin{aligned}
g= & g_{+}(x, y)+O\left(h^{\frac{1}{6}} \xi^{2} \frac{\partial G_{+}}{\partial \xi}\right)+O\left(h^{-\frac{1}{6}+\frac{1}{57}} \ln \frac{1}{h} \frac{G_{+}}{(1+|\xi|)}\right) \\
& +O\left(h^{-\frac{1}{6}+\frac{1}{57}} \ln \frac{1}{h} \frac{\partial G_{+}}{\partial \xi}\right)+O\left(h^{-\frac{1}{18}+\frac{20}{171}} \ln \frac{1}{h} \xi \frac{\partial G_{+}}{\partial \xi}\right)
\end{aligned}
$$

где

$$
\begin{gathered}
g_{+}(x, y)=\Omega_{+}^{2 / 3} \sqrt{2 \pi \tilde{x}_{+}} \frac{G_{+}(\xi, \eta)}{\sqrt{h}} \\
\xi=\left(\tilde{x}_{+}-x\right) \frac{\sqrt[3]{\Omega_{+}}}{h^{2 / 3}}, \quad \eta=y \frac{\sqrt[3]{\Omega_{+}}}{h^{2 / 3}}
\end{gathered}
$$

a

$$
\begin{aligned}
\Omega_{+}= & -U^{\prime}\left(\tilde{x}_{+}\right)+\frac{h^{1 / 3}}{\pi \tilde{x}_{+}} \int_{-\infty}^{\tilde{x}_{+}-\varepsilon}\left(1-\chi_{+}^{*}\left(x^{\prime}, \varepsilon\right)\right) \int_{-\infty}^{\infty}\left\{\left[\frac{1}{\left(\tilde{x}_{+}-x^{\prime}\right)} E\left(\frac{2 \sqrt{\tilde{x}_{+} x^{\prime}}}{\tilde{x}_{+}+x^{\prime}}\right)\right.\right. \\
& \left.\left.+\frac{1}{\left(\tilde{x}_{+}+x^{\prime}\right)} K\left(\frac{2 \sqrt{\tilde{x}_{+} x^{\prime}}}{\tilde{x}_{+}+x^{\prime}}\right)\right] \theta\left(x^{\prime}\right) g^{2}\left(x^{\prime}, y^{\prime}\right)-\frac{g_{+}^{2}\left(x^{\prime}, y^{\prime}\right)}{\tilde{x}_{+}-x^{\prime}}\right\} d y^{\prime} d x^{\prime} \\
& +O\left(h^{\frac{4}{9}+\frac{20}{171}} \ln \frac{1}{h}\right) .
\end{aligned}
$$


Здесь $\chi_{+}^{*}(x, \varepsilon) \stackrel{\text { def }}{=} \chi\left(\left(\tilde{x}_{+}-x\right) / 2 \varepsilon\right), h \rightarrow 0$. Тогда если $G_{+}(\xi, \eta)$ является решением (1.4), то после подстановки в (1.11) вместо $g$ функции $g_{+}$при $\left|x-\tilde{x}_{+}\right|<2 \varepsilon$ возникает невязка

$$
\begin{aligned}
& r_{+}=\left\{O\left(\left(\tilde{x}_{+}-x\right)^{2}\right)+O\left(h^{1+\frac{1}{57}} \ln \frac{1}{h}\right)+O\left(y^{2}\right)+O\left(h^{\frac{4}{9}+\frac{20}{171}} \ln \frac{1}{h}\left(\tilde{x}_{+}-x\right)\right)\right. \\
& +O\left(h^{1 / 3}\left(\tilde{x}_{+}-x\right)^{2} \int_{-\infty}^{\tilde{x}_{+}-\varepsilon} \frac{\left(1-\chi_{+}^{*}\left(x^{\prime}, \varepsilon\right)\right)}{\left(\tilde{x}_{+}-x^{\prime}\right)^{2}}\right. \\
& \left.\times \int_{-\infty}^{\infty}\left[\frac{\theta\left(x^{\prime}\right) g^{2}\left(x^{\prime}, y^{\prime}\right)}{\tilde{x}_{+}+x^{\prime}}-\frac{g_{+}^{2}\left(x^{\prime}, y^{\prime}\right)}{2 \tilde{x}_{+}}\right] d y^{\prime} d x^{\prime}\right) \\
& +O\left(h^{1 / 3} \int_{-\infty}^{\tilde{x}_{+}-\varepsilon}\left(1-\chi_{+}^{*}\left(x^{\prime}, \varepsilon\right)\right)\right. \\
& \left.\times \int_{-\infty}^{\infty} \ln \left[1+\frac{\left(y-y^{\prime}\right)^{2}}{\left(x-x^{\prime}\right)^{2}}\right]\left[\frac{\theta\left(x^{\prime}\right) g^{2}\left(x^{\prime}, y^{\prime}\right)}{\tilde{x}_{+}+x^{\prime}}-\frac{g_{+}^{2}\left(x^{\prime}, y^{\prime}\right)}{2 \tilde{x}_{+}}\right] d y^{\prime} d x^{\prime}\right) \\
& +O\left(h^{1 / 3} \int_{\tilde{x}_{+}-2 \varepsilon}^{\infty} \chi_{+}^{*}\left(x^{\prime}, \varepsilon\right)\right. \\
& \left.\times \int_{-\infty}^{\infty} \ln \left(\frac{\left(x-x^{\prime}\right)^{2}+\left(y-y^{\prime}\right)^{2}}{\left(\tilde{x}_{+}-x^{\prime}\right)^{2}}\right)\left(\frac{\tilde{x}_{+}-x^{\prime}}{\tilde{x}_{+}+x^{\prime}}\right) g_{+}^{2}\left(x^{\prime}, y^{\prime}\right) d y^{\prime} d x^{\prime}\right) \\
& +O\left(h^{1 / 3}\left(\tilde{x}_{+}-x\right) \int_{0}^{\infty} \frac{1}{\left(\tilde{x}_{+}+x^{\prime}\right)^{2}} \int_{-\infty}^{\infty} \ln \left(\frac{\left(x-x^{\prime}\right)^{2}+\left(y-y^{\prime}\right)^{2}}{\left(\tilde{x}_{+}-x^{\prime}\right)^{2}}\right)\right. \\
& \left.\times\left[\chi_{+}^{*}\left(x^{\prime}, \varepsilon\right) g_{+}^{2}\left(x^{\prime}, y^{\prime}\right)+\left(1-\chi_{+}^{*}\left(x^{\prime}, \varepsilon\right)\right) g^{2}\left(x^{\prime}, y^{\prime}\right)\right] d y^{\prime} d x^{\prime}\right) \\
& +O\left(h^{1 / 3} \int_{0}^{\infty} \int_{-\infty}^{\infty} \frac{\left(y-y^{\prime}\right)^{2}}{\left(\tilde{x}_{+}+x^{\prime}\right)^{3}}\left(1+|\ln | x-x^{\prime}||\right)\right. \\
& \left.\left.\times\left[\chi_{+}^{*}\left(x^{\prime}, \varepsilon\right) g_{+}^{2}\left(x^{\prime}, y^{\prime}\right)+\left(1-\chi_{+}^{*}\left(x^{\prime}, \varepsilon\right)\right) g^{2}\left(x^{\prime}, y^{\prime}\right)\right] d y^{\prime} d x^{\prime}\right)\right\} g_{+}(x, y) \text {. }
\end{aligned}
$$

Ниже выпишем формулы для асимптотик функций $G_{ \pm}(\xi, \eta)$ при $\xi \rightarrow+\infty, \eta=$ $O\left(\xi^{1 / 6}(\ln \xi)^{2 / 3}\right)$ и укажем значения входящих в них констант. Задание этих асимптотик позволяет выбрать экспоненциально убьвающие при $|\eta| \rightarrow+\infty$, а также при $\xi \rightarrow-\infty$ решения $G_{-}(\xi, \eta), G_{+}(\xi, \eta)$ уравнения $(1.4)$, через которые выражаются $g_{-}, g_{+} \cdot$

Для решений (1.4) в статье [1] при $\xi \rightarrow+\infty, \eta=O\left(\xi^{1 / 6}(\ln \xi)^{2 / 3}\right)$ были построены асимптотические разложения вида

$$
G(\xi, \eta)=\widetilde{T}(\xi, \eta) \cos (\widetilde{S}(\xi)+\widetilde{\varphi}(\xi, \eta))
$$

ЛЕмма 4.4 [1]. В формуле $(4.18)$

$$
\widetilde{S}(\xi)=S_{0}(\xi)+O\left(\xi^{-2 / 3}\right), \quad \xi \rightarrow+\infty
$$


əдe

$$
\begin{aligned}
& S_{0}(\xi)=\frac{2}{3} \xi^{3 / 2}-A_{-1} \sqrt{\xi}(2 \ln \xi-4)+2 A_{0} \sqrt{\xi}+3 \rho \tilde{k}^{2 / 3} \xi^{1 / 6}-\tilde{k} \pi^{2} A_{-1} \ln \xi \\
&+\delta-\frac{\sigma}{18 \tilde{k}^{2 / 3} \xi^{1 / 6}}+\frac{9 \sqrt{3}}{10} \frac{\pi \rho \tilde{k}^{5 / 3}}{\xi^{1 / 3}}+\frac{A_{-1}^{2}}{\sqrt{\xi}}\left[(\ln \xi)^{2}+4 \ln \xi+8\right] \\
&-\left[A_{0}+\frac{\tilde{k}^{2} \pi^{2}}{2}\right] \frac{A-1}{\sqrt{\xi}}(2 \ln \xi+4) \\
&-\left[\tilde{k}^{2} \pi^{2} A_{-1}+2 A_{1}-\frac{u}{243 \tilde{k}^{2}}-A_{0}^{2}\right] \frac{1}{\sqrt{\xi}}, \\
& A_{-1}=A_{-1}(\tilde{k}) \stackrel{\text { def }}{=} \int_{-\infty}^{\infty}\left\{\int_{-\infty}^{\infty} G^{2}\left(\xi^{\prime}, \eta^{\prime}\right) d \eta^{\prime}-\frac{\tilde{k} \theta\left(\xi^{\prime}\right)}{2 \sqrt{\xi^{\prime}}}\right\} d \xi^{\prime}, \\
& A_{0}=A_{0}(\tilde{k}) \stackrel{\text { def }}{=} \int_{-\infty}^{\infty} \ln \left|\xi^{\prime}\right|\left\{\int_{-\infty}^{\infty} G^{2}\left(\xi^{\prime}, \eta^{\prime}\right) d \eta^{\prime}-\frac{\tilde{k} \theta\left(\xi^{\prime}\right)}{2 \sqrt{\xi^{\prime}}}\right\} d \xi^{\prime} \\
& A_{1}=A_{1}(\tilde{k}) \stackrel{\text { def }}{=} \int_{-\infty}^{\infty}\left\{-\int_{0}^{\xi^{\prime}} \int_{-\infty}^{\infty} G^{2}\left(z, \eta^{\prime}\right) d \eta^{\prime} d z\right. \\
&+\theta\left(\xi^{\prime}\right) \frac{\tilde{k}}{2} \int_{0}^{\xi^{\prime}}\left[\frac{1}{\sqrt{z}}+\frac{A_{-1} \ln z}{z^{3 / 2}}-\frac{A_{0}}{z^{3 / 2}}-\frac{\rho \tilde{k}^{2 / 3}}{2 z^{11 / 6}}\right] d z \\
&\left.+\theta\left(\xi^{\prime}\right) \int_{1}^{\xi^{\prime}} \frac{\tilde{k}^{2} \pi^{2} A-1}{2 z^{2}} d z\right\} d \xi^{\prime} .
\end{aligned}
$$

Здесь $\delta$ - константа (сдвиг фазы), $\rho=\rho_{i}, \quad i=1,2, \ldots,-$ собственные значения задачи (3.6), (3.7), константы $\sigma=\sigma_{i}, \quad u=u_{i}$ задаются равенствами (3.24), (3.32), а функция $\theta$ - формулой (4.10).

Формулы для $\widetilde{T}$ и $\widetilde{\varphi}$ из (4.18) приведены в [1]. Они аналогичны построенным в $\S 2,3$ настоящей статьи разложениям для $T$ и $\varphi$ ( $T$ и $\varphi$ выражаются через $B$ и $I$ согласно формулам $(2.22),(2.23))$. Для однозначного задания асимптотики $G$ достаточно указать число $i$, упорядочивающее решения задачи $(3.6),(3.7)$, а также значение параметра $\tilde{k}$. В зависимости от того, вблизи от какой из точек $\tilde{x}_{-}, \tilde{x}_{+}$ строится асимптотика, будем вместо $\tilde{k}$ писать $\tilde{k}_{-}$или $\tilde{k}_{+}$.

Потребуем, чтобы решения уравнения (1.4) $G_{-}(\xi, \eta), G_{+}(\xi, \eta)$ при $\xi \rightarrow+\infty$, $\eta=O\left(\xi^{1 / 6}(\ln \xi)^{2 / 3}\right)$ имели асимптотики $(4.18)$ с тем же номером $i$, что и члены разложения (2.1). Кроме того, пусть для $G_{-}(\xi, \eta)$

$$
\tilde{k}_{-}=k /\left(2 \pi \tilde{x}_{-} \Omega_{-}^{4 / 3}\right)
$$

а для $G_{+}(\xi, \eta)$

$$
\tilde{k}_{+}=k /\left(2 \pi \tilde{x}_{+} \Omega_{+}^{4 / 3}\right) .
$$

Здесь $k$ - входящая в (2.1) константа (см.(2.19)), а $\Omega_{-}, \Omega_{+}$определены формулами (4.12), (4.16). 


\section{§5. Задача для фазы}

В этом параграфе мы подставим в равенства $(2.21),(2.28),(2.4),(4.12),(4.16)$ вблизи точек $\tilde{x}_{-}, \tilde{x}_{+}$вместо $g$ выражения для асимптотических решений $g_{-}, g_{+}$ и выразим возникающие интегралы через $\widetilde{S}^{\prime}(\xi)$ и входящие в $(4.20)$ константы. В результате будет получена задача для нахождения определяющей фазу ВКБ-разложения $(2.24)$ функции $S(x, h)$, а также констант $k, \tilde{x}_{-}, \tilde{x}_{+}, \Omega_{-}, \Omega_{+}$.

5.1. Преобразование соотношения (2.28). Пусть

$$
\begin{aligned}
H(\xi) \stackrel{\text { def }}{=} & \frac{1}{\sqrt{\xi}}+A_{-1} \frac{\ln \xi}{\xi^{3 / 2}}-\frac{A_{0}}{\xi^{3 / 2}}-\frac{\rho \tilde{k}^{2 / 3}}{2 \xi^{11 / 6}}+\frac{\tilde{k} \pi^{2} A_{-1}}{\xi^{2}}-\frac{\sigma}{108 \tilde{k}^{2 / 3} \xi^{13 / 6}} \\
& +\frac{3 \sqrt{3}}{10} \frac{\pi \rho \tilde{k}^{5 / 3}}{\xi^{7 / 3}}+\frac{3}{2} A_{-1}^{2} \frac{(\ln \xi)^{2}}{\xi^{5 / 2}}-\left[3 A_{0}+\frac{\tilde{k}^{2} \pi^{2}}{2}\right] A_{-1} \frac{\ln \xi}{\xi^{5 / 2}} \\
& -\frac{1}{2 \xi^{5 / 2}}\left[\tilde{k}^{2} \pi^{2} A_{-1}+2 A_{1}-\frac{u}{243 \tilde{k}^{2}}-3 A_{0}^{2}\right] .
\end{aligned}
$$

Тогда в силу (4.19), (4.20) и формулы Тейлора имеем $1 / \widetilde{S}^{\prime}(\xi)=H(\xi)+O\left(\xi^{-8 / 3}\right)$, $\xi \rightarrow+\infty$. Функция $H(\xi)$ в отличие от $1 / S_{0}^{\prime}(\xi)$ не имеет особенностей при $\xi>0$.

Определим

$$
\begin{aligned}
D_{-1}\left(\tilde{k}_{ \pm}\right) & \stackrel{\text { def }}{=} A_{-1}\left(\tilde{k}_{ \pm}\right)+\frac{\tilde{k}_{ \pm}}{2} \int_{0}^{\infty}\left(\frac{1}{\sqrt{\xi}}-\theta(\xi-1) H(\xi)\right) d \xi \\
& =\int_{-\infty}^{\infty}\left(\int_{-\infty}^{\infty} G_{ \pm}^{2}(\xi, \eta) d \eta-\frac{\tilde{k}_{ \pm}}{2} \theta(\xi-1) H(\xi)\right) d \xi
\end{aligned}
$$

ЛЕмма 5.1. При $h \rightarrow 0$ справедливы равенства

$$
\begin{aligned}
& \int_{0}^{\tilde{x}_{-}+\varepsilon} \chi_{-}(x, \varepsilon) \int_{-\infty}^{\infty} g_{-}^{2}(x, y) d y d x \\
& =h^{1 / 3} \Omega_{-}^{2 / 3} 2 \pi \tilde{x}_{-}\left[D_{-1}\left(\tilde{k}_{-}\right)+\int_{1}^{e_{-}} \frac{\tilde{k}_{-}}{2} \chi\left(\frac{\xi}{e_{-}}\right) H(\xi) d \xi\right]+O\left(\frac{h^{13 / 9}}{\varepsilon^{5 / 3}}\right) \\
& \int_{\tilde{x}_{+}-\varepsilon}^{\infty} \chi_{+}(x, \varepsilon) \int_{-\infty}^{\infty} g_{+}^{2}(x, y) d y d x \\
& =h^{1 / 3} \Omega_{+}^{2 / 3} 2 \pi \tilde{x}_{+}\left[D_{-1}\left(\tilde{k}_{+}\right)+\int_{1}^{e_{+}} \frac{\tilde{k}_{+}}{2} \chi\left(\frac{\xi}{e_{+}}\right) H(\xi) d \xi\right]+O\left(\frac{h^{13 / 9}}{\varepsilon^{5 / 3}}\right)
\end{aligned}
$$

¿əe

$$
e_{ \pm}=\varepsilon \sqrt[3]{\Omega_{ \pm}} / h^{2 / 3}
$$

а $\chi=\chi(\xi) \in C^{\infty}\left(\mathbb{R}^{1}\right)$ - неотрицательная функиия такая, что $\chi(\xi) \equiv 1 \mathrm{nри}$ $\xi<1 / 2 u \chi(\xi) \equiv 0$ при $\xi>1$.

С учетом (5.3), (5.4) соотношение (2.28) принимает вид

$$
h^{1 / 3} \Omega_{-}^{2 / 3} 2 \pi \tilde{x}_{-}\left[D_{-1}\left(\tilde{k}_{-}\right)+\int_{1}^{e_{-}} \frac{\tilde{k}_{-}}{2} \chi\left(\frac{\xi}{e_{-}}\right) H(\xi) d \xi\right]
$$




$$
\begin{aligned}
& +\int_{\tilde{x}_{-}+\varepsilon / 2}^{\tilde{x}_{+}-\varepsilon / 2}\left(1-\chi_{-}(x, \varepsilon)-\chi_{+}(x, \varepsilon)\right) \frac{k}{2 S^{\prime}(x, h)} d x \\
& +h^{1 / 3} \Omega_{+}^{2 / 3} 2 \pi \tilde{x}_{+}\left[D_{-1}\left(\tilde{k}_{+}\right)+\int_{1}^{e+} \frac{\tilde{k}_{+}}{2} \chi\left(\frac{\xi}{e_{+}}\right) H(\xi) d \xi\right]=1+O\left(\frac{h^{13 / 9}}{\varepsilon^{5 / 3}}\right) \\
& +O\left(\int_{\tilde{x}_{-}+\varepsilon / 2}^{\tilde{x}_{+}-\varepsilon / 2}\left(1-\chi_{-}(x, \varepsilon)-\chi_{+}(x, \varepsilon)\right)\right. \\
& \left.\times \int_{-\infty}^{\infty} T^{2}\left(x, \tau_{1}, h\right) \cos 2 \Phi\left(x, \tau_{1}, h\right) d \tau_{1} d x\right), \quad h \rightarrow 0
\end{aligned}
$$

Исключая из (5.6) разбиение единицы, а также интегрируя по частям в содержащем $\cos 2 \Phi$ интеграле, окончательно имеем

$$
\begin{aligned}
& h^{1 / 3} \Omega_{-}^{2 / 3} 2 \pi \tilde{x}_{-}\left[D_{-1}\left(\tilde{k}_{-}\right)+\int_{1}^{e_{-}} \frac{\tilde{k}_{-}}{2} H(\xi) d \xi\right]+\int_{\tilde{x}_{-}+\varepsilon}^{\tilde{x}_{+}-\varepsilon} \frac{k d x}{2 S^{\prime}(x, h)} \\
& \quad+h^{1 / 3} \Omega_{+}^{2 / 3} 2 \pi \tilde{x}_{+}\left[D_{-1}\left(\tilde{k}_{+}\right)+\int_{1}^{e_{+}} \frac{\tilde{k}_{+}}{2} H(\xi) d \xi\right]=1+O\left(h^{\frac{2}{3}+\frac{1}{57}}\right), \quad h \rightarrow 0 .
\end{aligned}
$$

5.2. Преобразование соотношений $(2.4)$. В силу (2.6) при $x \rightarrow \tilde{x}_{ \pm}$получаем

$$
\frac{1}{\tilde{x}_{ \pm}+x} K\left(\frac{2 \sqrt{\tilde{x}_{ \pm} x}}{\tilde{x}_{ \pm}+x}\right)=-\frac{1}{2 \tilde{x}_{ \pm}} \ln \left|x-\tilde{x}_{ \pm}\right|+\frac{\ln \left(8 \tilde{x}_{ \pm}\right)}{2 \tilde{x}_{ \pm}}+O\left(\left(x-\tilde{x}_{ \pm}\right) \ln \left|x-\tilde{x}_{ \pm}\right|\right) .
$$

Поэтому (2.4) можно записать в виде

$$
\begin{aligned}
& U\left(\tilde{x}_{-}\right)+\frac{2}{\pi} h^{1 / 3}\left\{-\frac{1}{2 \tilde{x}_{-}} \int_{0}^{\tilde{x}_{-}+\varepsilon} \chi_{-}(x, \varepsilon) \ln \left|x-\tilde{x}_{-}\right| \int_{-\infty}^{\infty} g_{-}^{2}(x, y) d y d x\right. \\
& +\frac{\ln \left(8 \tilde{x}_{-}\right)}{2 \tilde{x}_{-}} \int_{0}^{\tilde{x}_{-}+\varepsilon} \chi_{-}(x, \varepsilon) \int_{-\infty}^{\infty} g_{-}^{2}(x, y) d y d x+O\left(\varepsilon^{3 / 2} \ln \frac{1}{h}\right) \\
& +\int_{\tilde{x}_{-}+\varepsilon / 2}^{\tilde{x}_{+}-\varepsilon / 2} \frac{\left(1-\chi_{-}(x, \varepsilon)-\chi_{+}(x, \varepsilon)\right)}{\left(\tilde{x}_{-}+x\right)} K\left(\frac{2 \sqrt{\tilde{x}_{-} x}}{\tilde{x}_{-}+x}\right) \frac{k d x}{2 S^{\prime}(x, h)} \\
& \left.+\frac{1}{\tilde{x}_{+}+\tilde{x}_{-}} K\left(\frac{2 \sqrt{\tilde{x}_{+} \tilde{x}_{-}}}{\tilde{x}_{+}+\tilde{x}_{-}}\right) \int_{\tilde{x}_{+-\varepsilon}}^{\infty} \chi_{+}(x, \varepsilon) \int_{-\infty}^{\infty} g_{+}^{2}(x, y) d y d x+O\left(\varepsilon^{3 / 2}\right)\right\} \\
& =O\left(h^{1+\frac{1}{57}} \ln \frac{1}{h}\right)+O\left(h^{1 / 3} \int_{\tilde{x}_{-}+\varepsilon / 2}^{\tilde{x}_{+}-\varepsilon / 2} \frac{\left(1-\chi_{-}(x, \varepsilon)-\chi_{+}(x, \varepsilon)\right)}{\left(\tilde{x}_{-}+x\right)}\right. \\
& \left.\times K\left(\frac{2 \sqrt{\tilde{x}_{-} x}}{\tilde{x}_{-}+x}\right) \int_{-\infty}^{\infty} T^{2}\left(x, \tau_{1}, h\right) \cos 2 \Phi\left(x, \tau_{1}, h\right) d \tau_{1} d x\right), \quad h \rightarrow 0, \\
& U\left(\tilde{x}_{+}\right)+\frac{2}{\pi} h^{1 / 3}\left\{\frac{1}{\tilde{x}_{+}+\tilde{x}_{-}} K\left(\frac{2 \sqrt{\tilde{x}_{+} \tilde{x}_{-}}}{\tilde{x}_{+}+\tilde{x}_{-}}\right) \int_{0}^{\tilde{x}_{-}+\varepsilon} \chi_{-}(x, \varepsilon)\right. \\
& \times \int_{-\infty}^{\infty} g_{-}^{2}(x, y) d y d x+O\left(\varepsilon^{3 / 2}\right)
\end{aligned}
$$




$$
\begin{aligned}
& +\int_{\tilde{x}_{-}+\varepsilon / 2}^{\tilde{x}_{+}-\varepsilon / 2} \frac{\left(1-\chi_{-}(x, \varepsilon)-\chi_{+}(x, \varepsilon)\right)}{\left(\tilde{x}_{+}+x\right)} K\left(\frac{2 \sqrt{\tilde{x}_{+} x}}{\tilde{x}_{+}+x}\right) \frac{k d x}{2 S^{\prime}(x, h)} \\
& -\frac{1}{2 \tilde{x}_{+}} \int_{\tilde{x}_{+}-\varepsilon}^{\infty} \chi_{+}(x, \varepsilon) \ln \left|x-\tilde{x}_{+}\right| \int_{-\infty}^{\infty} g_{+}^{2}(x, y) d y d x \\
& \left.+\frac{\ln \left(8 \tilde{x}_{+}\right)}{2 \tilde{x}_{+}} \int_{\tilde{x}_{+}-\varepsilon}^{\infty} \chi_{+}(x, \varepsilon) \int_{-\infty}^{\infty} g_{+}^{2}(x, y) d y d x+O\left(\varepsilon^{3 / 2} \ln \frac{1}{h}\right)\right\} \\
& =O\left(h^{1+\frac{1}{57}} \ln \frac{1}{h}\right)+O\left(h^{1 / 3} \int_{\tilde{x}_{-}+\varepsilon / 2}^{\tilde{x}_{+}-\varepsilon / 2} \frac{\left(1-\chi_{-}(x, \varepsilon)-\chi_{+}(x, \varepsilon)\right)}{\left(\tilde{x}_{+}+x\right)}\right. \\
& \left.\times K\left(\frac{2 \sqrt{\tilde{x}_{+} x}}{\tilde{x}_{+}+x}\right) \int_{-\infty}^{\infty} T^{2}\left(x, \tau_{1}, h\right) \cos 2 \Phi\left(x, \tau_{1}, h\right) d \tau_{1} d x\right), \quad h \rightarrow 0 .
\end{aligned}
$$

Определим

$$
\begin{aligned}
D_{0}\left(\tilde{k}_{ \pm}\right) & \stackrel{\text { def }}{=} A_{0}\left(\tilde{k}_{ \pm}\right)+\frac{\tilde{k}_{ \pm}}{2} \int_{0}^{\infty} \ln \xi\left(\frac{1}{\sqrt{\xi}}-\theta(\xi-1) H(\xi)\right) d \xi \\
& =\int_{-\infty}^{\infty} \ln |\xi|\left(\int_{-\infty}^{\infty} G_{ \pm}^{2}(\xi, \eta) d \eta-\frac{\tilde{k}_{ \pm}}{2} \theta(\xi-1) H(\xi)\right) d \xi
\end{aligned}
$$

ЛЕммА 5.2. При $h \rightarrow 0$ справедливы равенства

$$
\begin{aligned}
\int_{0}^{\tilde{x}_{-}+\varepsilon} \chi_{-}(x, \varepsilon) \ln \left|x-\tilde{x}_{-}\right| \int_{-\infty}^{\infty} g_{-}^{2}(x, y) d y d x \\
=h^{1 / 3} \Omega_{-}^{2 / 3} 2 \pi \tilde{x}_{-}\left\{D_{0}\left(\tilde{k}_{-}\right)+\int_{1}^{e_{-}} \frac{\tilde{k}_{-}}{2} \chi\left(\frac{\xi}{e_{-}}\right)(\ln \xi) H(\xi) d \xi\right. \\
\left.+\ln \left|\frac{h^{2 / 3}}{\sqrt[3]{\Omega_{-}}}\right| \cdot\left[D_{-1}\left(\tilde{k}_{-}\right)+\int_{1}^{e} \frac{\tilde{k}_{-}}{2} \chi\left(\frac{\xi}{e_{-}}\right) H(\xi) d \xi\right]\right\} \\
+O\left(\frac{h^{13 / 9}}{\varepsilon^{5 / 3}} \ln \frac{1}{h}\right), \\
\int_{\tilde{x}_{+-} \varepsilon \quad}^{\infty} \chi_{+}(x, \varepsilon) \ln \left|x-\tilde{x}_{+}\right| \int_{-\infty}^{\infty} g_{+}^{2}(x, y) d y d x \\
=h^{1 / 3} \Omega_{+}^{2 / 3} 2 \pi \tilde{x}_{+}\left\{D_{0}\left(\tilde{k}_{+}\right)+\int_{1}^{e_{+}} \frac{\tilde{k}_{+}}{2} \chi\left(\frac{\xi}{e_{+}}\right)(\ln \xi) H(\xi) d \xi\right. \\
\left.+\ln \left|\frac{h^{2 / 3}}{\sqrt[3]{\Omega_{+}}}\right| \cdot\left[D_{-1}\left(\tilde{k}_{+}\right)+\int_{1}^{e_{+}} \frac{\tilde{k}_{+}}{2} \chi\left(\frac{\xi}{e_{+}}\right) H(\xi) d \xi\right]\right\} \\
+O\left(\frac{h^{13 / 9}}{\varepsilon^{5 / 3}} \ln \frac{1}{h}\right) .
\end{aligned}
$$

Далее подставим (5.3), (5.4), (5.10), (5.11) в (5.8), (5.9), исключим разбиение единищы и проинтегрируем по частям в содержащих $\cos 2 \Phi$ интегралах. Окончательно имеем

$$
U\left(\tilde{x}_{-}\right)+2 h^{1 / 3}\left\{-h^{1 / 3} \Omega_{-}^{2 / 3}\left[D_{0}\left(\tilde{k}_{-}\right)+\int_{1}^{e} \frac{\tilde{k}_{-}}{2}(\ln \xi) H(\xi) d \xi\right]\right.
$$




$$
\begin{aligned}
& +h^{1 / 3} \Omega_{-}^{2 / 3} \ln \left|\frac{8 \tilde{x}_{-} \sqrt[3]{\Omega_{-}}}{h^{2 / 3}}\right| \cdot\left[D_{-1}\left(\tilde{k}_{-}\right)+\int_{1}^{e_{-}} \frac{\tilde{k}_{-}}{2} H(\xi) d \xi\right] \\
& +\int_{\tilde{x}_{-}+\varepsilon}^{\tilde{x}_{+}-\varepsilon} \frac{1}{\pi\left(\tilde{x}_{-}+x\right)} K\left(\frac{2 \sqrt{\tilde{x}_{-} x}}{\tilde{x}_{-}+x}\right) \frac{k d x}{2 S^{\prime}(x, h)} \\
& \left.+\frac{h^{1 / 3} \Omega_{+}^{2 / 3} 2 \tilde{x}_{+}}{\tilde{x}_{+}+\tilde{x}_{-}} K\left(\frac{2 \sqrt{\tilde{x}_{+} \tilde{x}_{-}}}{\tilde{x}_{+}+\tilde{x}_{-}}\right)\left[D_{-1}\left(\tilde{k}_{+}\right)+\int_{1}^{e_{+}} \frac{\tilde{k}_{+}}{2} H(\xi) d \xi\right]\right\} \\
& =O\left(h^{1+\frac{1}{57}} \ln \frac{1}{h}\right), \quad h \rightarrow 0, \\
& U\left(\tilde{x}_{+}\right)+2 h^{1 / 3}\left\{\frac{h^{1 / 3} \Omega_{-}^{2 / 3} 2 \tilde{x}_{-}}{\tilde{x}_{+}+\tilde{x}_{-}} K\left(\frac{2 \sqrt{\tilde{x}_{+} \tilde{x}_{-}}}{\tilde{x}_{+}+\tilde{x}_{-}}\right)\left[D_{-1}\left(\tilde{k}_{-}\right)+\int_{1}^{e_{-}} \frac{\tilde{k}_{-}}{2} H(\xi) d \xi\right]\right. \\
& +\int_{\tilde{x}_{-}+\varepsilon}^{\tilde{x}_{+}-\varepsilon} \frac{1}{\pi\left(\tilde{x}_{+}+x\right)} K\left(\frac{2 \sqrt{\tilde{x}_{+} x}}{\tilde{x}_{+}+x}\right) \frac{k d x}{2 S^{\prime}(x, h)} \\
& -h^{1 / 3} \Omega_{+}^{2 / 3}\left[D_{0}\left(\tilde{k}_{+}\right)+\int_{1}^{e_{+}} \frac{\tilde{k}_{+}}{2}(\ln \xi) H(\xi) d \xi\right] \\
& \left.+h^{1 / 3} \Omega_{+}^{2 / 3} \ln \left|\frac{8 \tilde{x}_{+} \sqrt[3]{\Omega_{+}}}{h^{2 / 3}}\right|\left[D_{-1}\left(\tilde{k}_{+}\right)+\int_{1}^{e_{+}} \frac{\tilde{k}_{+}}{2} H(\xi) d \xi\right]\right\} \\
& =O\left(h^{1+\frac{1}{57}} \ln \frac{1}{h}\right), \quad h \rightarrow 0 \text {. }
\end{aligned}
$$

Здесь $e_{ \pm}$определены формулой (5.5).

5.3. Преобразование соотношений (4.12), (4.16).

ЛЕмма 5.3. При $h \rightarrow 0$ справедливы равенства

$$
\begin{aligned}
\Omega_{-}=U^{\prime}\left(\tilde{x}_{-}\right)+h^{1 / 3} \int_{\tilde{x}_{-} \varepsilon}^{\infty}\left\{\left[\frac{1}{x-\tilde{x}_{-}} E\left(\frac{2 \sqrt{\tilde{x}_{-} x}}{\tilde{x}_{-}+x}\right)\right.\right. \\
\left.\quad-\frac{1}{\tilde{x}_{-}+x} K\left(\frac{2 \sqrt{\tilde{x}_{-} x}}{\tilde{x}_{-}+x}\right)\right] \frac{k \theta\left(\tilde{x}_{+}-\varepsilon-x\right)}{2 \pi \tilde{x}_{-} S^{\prime}(x, h)} \\
\left.\quad-\frac{\tilde{k}_{-} \Omega_{-}}{\left(x-\tilde{x}_{-}\right) h^{1 / 3}} H\left(\frac{\left(x-\tilde{x}_{-}\right) \sqrt[3]{\Omega_{-}}}{h^{2 / 3}}\right)\right\} d x+O\left(h^{\frac{4}{9}+\frac{20}{171}} \ln \frac{1}{h}\right), \\
\Omega_{+}=-U^{\prime}\left(\tilde{x}_{+}\right)+h^{1 / 3} \int_{-\infty}^{\tilde{x}_{+}-\varepsilon}\left\{\left[\frac{1}{\tilde{x}_{+}-x} E\left(\frac{2 \sqrt{\tilde{x}_{+} x}}{\tilde{x}_{+}+x}\right)\right.\right. \\
\left.\quad+\frac{1}{\tilde{x}_{+}+x} K\left(\frac{2 \sqrt{\tilde{x}_{+} x}}{\tilde{x}_{+}+x}\right)\right] \frac{k \theta\left(x-\tilde{x}_{-}+\varepsilon\right)}{2 \pi \tilde{x}_{+} S^{\prime}(x, h)} \\
\left.\quad-\frac{\tilde{k}_{+} \Omega_{+}}{\left(\tilde{x}_{+}-x\right) h^{1 / 3}} H\left(\frac{\left(\tilde{x}_{+}-x\right) \sqrt[3]{\Omega_{+}}}{h^{2 / 3}}\right)\right\} d x+O\left(h^{\frac{4}{9}+\frac{20}{171}} \ln \frac{1}{h}\right) .
\end{aligned}
$$


5.4. Преобразование уравнения (2.21). Подставим в формулы (2.14) для $j_{-}$и $j_{+}$вместо $g$ выражения для $g_{-}$и $g_{+}$соответственно. Так как в силу (4.5)-(4.7)

$$
\begin{aligned}
\frac{2}{\pi\left(x+x^{\prime}\right)} K\left(\frac{2 \sqrt{x x^{\prime}}}{x+x^{\prime}}\right)= & \frac{2}{\pi\left(x+\tilde{x}_{-}\right)} K\left(\frac{2 \sqrt{x \tilde{x}_{-}}}{\tilde{x}_{-}+x}\right)-\frac{2}{\pi\left(x+\tilde{x}_{-}\right)} \ln \left|\frac{x-x^{\prime}}{x-\tilde{x}_{-}}\right| \\
& +O\left(\left(x^{\prime}-\tilde{x}_{-}\right)\left(1+|\ln | x-\tilde{x}_{-}||\right)\right), \quad x^{\prime} \rightarrow \tilde{x}_{-},
\end{aligned}
$$

то при $x \in\left(\tilde{x}_{-}+\varepsilon, \tilde{x}_{+}-\varepsilon\right)$ имеем

$$
\begin{aligned}
\int_{0}^{\tilde{x}_{-}+\varepsilon} & \frac{2}{\pi\left(x+x^{\prime}\right)} K\left(\frac{2 \sqrt{x x^{\prime}}}{x+x^{\prime}}\right) \chi_{-}\left(x^{\prime}, \varepsilon\right) \int_{-\infty}^{\infty} g_{-}^{2}\left(x^{\prime}, y^{\prime}\right) d y^{\prime} d x^{\prime} \\
= & \frac{2}{\pi\left(x+\tilde{x}_{-}\right)}\left\{\left[K\left(\frac{2 \sqrt{x \tilde{x}_{-}}}{x+\tilde{x}_{-}}\right)+\ln \left|x-\tilde{x}_{-}\right|\right]\right. \\
& \times \int_{0}^{\tilde{x}_{-}+\varepsilon} \chi_{-}\left(x^{\prime}, \varepsilon\right) \int_{-\infty}^{\infty} g_{-}^{2}\left(x^{\prime}, y^{\prime}\right) d y^{\prime} d x^{\prime} \\
& -\int_{0}^{\tilde{x}_{-}+\varepsilon} \chi_{-}\left(x^{\prime}, \varepsilon\right) \ln \left|x^{\prime}-\tilde{x}_{-}\right| \int_{-\infty}^{\infty} g_{-}^{2}\left(x^{\prime}, y^{\prime}\right) d y^{\prime} d x^{\prime} \\
& \left.-2 \pi \tilde{x}_{-} h^{1 / 3} \Omega_{-}^{2 / 3} \int_{-\infty}^{e_{-}} \chi\left(\frac{\xi^{\prime}}{e_{-}}\right) \ln \left|1-\frac{\xi}{\xi^{\prime}}\right| \int_{-\infty}^{\infty} G_{-}^{2}\left(\xi^{\prime}, \eta^{\prime}\right) d \eta^{\prime} d \xi^{\prime}\right\} \\
& +O\left(\varepsilon^{3 / 2} \ln \frac{1}{h}\right) .
\end{aligned}
$$

Определим

$$
\begin{aligned}
D_{1}\left(\tilde{k}_{ \pm}\right) \stackrel{\text { def }}{=} & A_{1}\left(\tilde{k}_{ \pm}\right)+\frac{\tilde{k}_{ \pm}}{2} \int_{0}^{\infty}\left\{\int_{1}^{\xi} H(z) d z\right. \\
& -\int_{0}^{\xi}\left[\frac{1}{\sqrt{z}}+A_{-1}\left(\tilde{k}_{ \pm}\right) \frac{\ln z}{z^{3 / 2}}-\frac{A_{0}\left(\tilde{k}_{ \pm}\right)}{z^{3 / 2}}-\frac{\rho \tilde{k}_{ \pm}^{2 / 3}}{2 z^{11 / 6}}\right] d z \\
& \left.-\int_{1}^{\xi} \tilde{k}_{ \pm} \pi^{2} A_{-1}\left(\tilde{k}_{ \pm}\right) \frac{d z}{z^{2}}\right\} d \xi \\
= & \int_{-\infty}^{\infty}\left\{-\int_{0}^{\xi} \int_{-\infty}^{\infty} G_{ \pm}^{2}(z, \eta) d \eta d z+\frac{\tilde{k}_{ \pm}}{2} \theta(\xi) \int_{1}^{\xi} H(z) d z\right\} d \xi
\end{aligned}
$$

Лемма 5.4. Пусть $\xi>e_{-}, e_{-} \rightarrow+\infty$. Тогда справедливо равенство

$$
\begin{aligned}
\int_{-\infty}^{e_{-}} \chi & \left(\frac{\xi^{\prime}}{e_{-}}\right) \ln \left|1-\frac{\xi}{\xi^{\prime}}\right| \int_{-\infty}^{\infty} G_{-}^{2}\left(\xi^{\prime}, \eta^{\prime}\right) d \eta^{\prime} d \xi^{\prime} \\
= & \int_{1}^{e_{-}} \chi\left(\frac{\xi^{\prime}}{e_{-}}\right) \ln \left|1-\frac{\xi}{\xi^{\prime}}\right| \frac{\tilde{k}_{-}}{2} H\left(\xi^{\prime}\right) d \xi^{\prime}+D_{-1}\left(\tilde{k}_{-}\right) \ln \xi \\
& \quad-D_{0}\left(\tilde{k}_{-}\right)-D_{1}\left(\tilde{k}_{-}\right) / \xi+O\left(\left(1+\ln \left|\xi / e_{-}\right|\right) / \varepsilon_{-}^{5 / 3}\right) .
\end{aligned}
$$


Из соотношений $(5.3),(5.10),(5.16)-(5.18)$ вытекает, что при $x \in\left(\tilde{x}_{-}+\varepsilon, \tilde{x}_{+}-\varepsilon\right)$

$$
\begin{aligned}
\int_{0}^{\tilde{x}_{-}+\varepsilon} & \frac{2}{\pi\left(x+x^{\prime}\right)} K\left(\frac{2 \sqrt{x x^{\prime}}}{x+x^{\prime}}\right) \chi_{-}\left(x^{\prime}, \varepsilon\right) \int_{-\infty}^{\infty} g_{-}^{2}\left(x^{\prime}, y^{\prime}\right) d y^{\prime} d x^{\prime} \\
= & \frac{4 \tilde{x}_{-} h^{1 / 3} \Omega_{-}^{2 / 3}}{x+\tilde{x}_{-}}\left\{K\left(\frac{2 \sqrt{x \tilde{x}_{-}}}{x+\tilde{x}_{-}}\right) D_{-1}\left(\tilde{k}_{-}\right)+\frac{D_{1}\left(\tilde{k}_{-}\right)}{\xi}\right. \\
& \left.+\int_{1}^{e_{-}}\left[K\left(\frac{2 \sqrt{x \tilde{x}_{-}}}{x+\tilde{x}_{-}}\right)+\ln \left|\frac{x-\tilde{x}_{-}}{x-x^{\prime}}\right|\right] \frac{\tilde{k}_{-}}{2} \chi\left(\frac{\xi^{\prime}}{e_{-}}\right) H\left(\xi^{\prime}\right) d \xi^{\prime}\right\} \\
& +O\left(\frac{h^{13 / 9}}{\varepsilon^{5 / 3}} \ln \frac{1}{h}\right)+O\left(\varepsilon^{3 / 2} \ln \frac{1}{h}\right) \\
= & \frac{2 \pi \tilde{x}_{-} \Omega_{-}}{h^{1 / 3}} \int_{\tilde{x}_{-}+h^{2 / 3} / \sqrt[3]{\Omega_{-}}} \frac{\chi-\left(x^{\prime}, \varepsilon\right)}{\pi\left(x+x^{\prime}\right)} K\left(\frac{2 \sqrt{x x^{\prime}}}{x+x^{\prime}}\right) \tilde{k}_{-} H\left(\frac{\left(x^{\prime}-\tilde{x}_{-}\right) \sqrt[3]{\Omega_{-}}}{h^{2 / 3}}\right) d x^{\prime} \\
& +\frac{4 \tilde{x}_{-}}{x+\tilde{x}_{-}}\left\{h^{1 / 3} \Omega_{-}^{2 / 3} K\left(\frac{2 \sqrt{x \tilde{x}_{-}}}{x+\tilde{x}_{-}}\right) D\left(\tilde{k}_{-}\right)+\frac{h \sqrt[3]{\Omega_{-}} D_{1}\left(\tilde{k}_{-}\right)}{x-\tilde{x}_{-}}\right\} \\
& +O\left(\frac{h^{13 / 9}}{\varepsilon^{5 / 3}} \ln \frac{1}{h}\right)+O\left(\varepsilon^{3 / 2} \ln \frac{1}{h}\right), \quad h \rightarrow 0 .
\end{aligned}
$$

Аналогично доказывается, что

$$
\begin{aligned}
\int_{\tilde{x}_{+}-\varepsilon}^{\infty} & \frac{2}{\pi\left(x+x^{\prime}\right)} K\left(\frac{2 \sqrt{x x^{\prime}}}{x+x^{\prime}}\right) \chi_{+}\left(x^{\prime}, \varepsilon\right) \int_{-\infty}^{\infty} g_{+}^{2}\left(x^{\prime}, y^{\prime}\right) d y^{\prime} d x^{\prime} \\
= & \frac{2 \pi \tilde{x}_{+} \Omega_{+}}{h^{1 / 3}} \int_{\tilde{x}_{+}-\varepsilon}^{\tilde{x}_{+}-h^{2 / 3} / \sqrt[3]{\Omega_{+}}} \frac{\chi_{+}\left(x^{\prime}, \varepsilon\right)}{\pi\left(x+x^{\prime}\right)} K\left(\frac{2 \sqrt{x x^{\prime}}}{x+x^{\prime}}\right) \tilde{k}_{+} H\left(\frac{\left(\tilde{x}_{+}-x^{\prime}\right) \sqrt[3]{\Omega_{+}}}{h^{2 / 3}}\right) d x^{\prime} \\
& +\frac{4 \tilde{x}_{+}}{x+\tilde{x}_{+}}\left\{h^{1 / 3} \Omega_{+}^{2 / 3} K\left(\frac{2 \sqrt{x \tilde{x}_{+}}}{x+\tilde{x}_{+}}\right) D_{-1}\left(\tilde{k}_{+}\right)+\frac{h \sqrt[3]{\Omega_{+}} D_{1}\left(\tilde{k}_{+}\right)}{\tilde{x}_{+}-x}\right\} \\
& +O\left(\frac{h^{13 / 9}}{\varepsilon^{5 / 3}} \ln \frac{1}{h}\right)+O\left(\varepsilon^{3 / 2} \ln \frac{1}{h}\right), \quad h \rightarrow 0
\end{aligned}
$$

Далее воспользуемся тем, что в разложении $h^{8 / 9} L(x, h)=h^{8 / 9} \ell_{0}+h^{10 / 9} \ell_{1}+$ $h^{4 / 3} \ell_{2}+O\left(h^{13 / 9} \ell_{3}\right)$ перед $\ell_{1}, \ell_{2}$ стоят множители $h^{\mu}$, где $\mu>1$. Мы заменим эти функции на главные члены асимптотик. Главные члены имеют особенности при $x \rightarrow \tilde{x}_{ \pm}$. Остальные, более гладкие, слагаемые войдут в остаточный член. Из (3.23), (3.31) получаем

$$
\begin{aligned}
\ell_{1}(x)= & \frac{\sigma}{54 k^{2 / 3}}\left[\frac{\left(2 \pi \tilde{x}_{-}\right)^{2 / 3}\left(U^{\prime}\left(\tilde{x}_{-}\right)\right)^{4 / 3}}{\left(x-\tilde{x}_{-}\right)^{2 / 3}}+\frac{\left(2 \pi \tilde{x}_{+}\right)^{2 / 3}\left|U^{\prime}\left(\tilde{x}_{+}\right)\right|^{4 / 3}}{\left(\tilde{x}_{+}-x\right)^{2 / 3}}\right] \\
& +O\left(\frac{h^{1 / 3}}{\left(x-\tilde{x}_{-}\right)^{7 / 6}}\right)+O\left(\frac{h^{1 / 3}}{\left(\tilde{x}_{+}-x\right)^{7 / 6}}\right)+O(1), \\
\ell_{2}(x)= & -\frac{u}{243 k^{2}}\left\{\frac{\left(2 \pi \tilde{x}_{-}\right)^{2}\left(U^{\prime}\left(\tilde{x}_{-}\right)\right)^{3}}{x-\tilde{x}_{-}}+\frac{\left(2 \pi \tilde{x}_{+}\right)^{2}\left|U^{\prime}\left(\tilde{x}_{+}\right)\right|^{3}}{\tilde{x}_{+}-x}\right\} \\
& +O\left(\frac{h^{1 / 9}}{\left(x-\tilde{x}_{-}\right)^{7 / 6}}\right)+O\left(\frac{h^{1 / 9}}{\left(\tilde{x}_{+}-x\right)^{7 / 6}}\right)+O(1) .
\end{aligned}
$$


Остается подставить соотношения (3.10), (5.19)-(5.22) в (2.21) и заменить $1 / S^{\prime}\left(x^{\prime}, h\right)$ на $H\left(\xi^{\prime}\right)$ при $x^{\prime} \in\left(\tilde{x}_{+}-\varepsilon, \tilde{x}_{+}-\varepsilon / 2\right), x^{\prime} \in\left(\tilde{x}_{-}+\varepsilon / 2, \tilde{x}_{-}+\varepsilon\right)$.

Справедлива

Теорема 5.1. Функиия $S^{\prime}(x, h)$ на интервале $x \in\left(\tilde{x}_{-}+\varepsilon, \tilde{x}_{+}-\varepsilon\right)$ удовлетворяет уравнению

$$
\begin{aligned}
-\left(S^{\prime}(x, h)\right)^{2}+U(x) & +\Omega_{-} \int_{\tilde{x}_{-}+h^{2 / 3} / \sqrt[3]{\Omega_{-}}}^{\tilde{x}_{-}+\varepsilon} \frac{2 \tilde{x}_{-}}{\left(x+x^{\prime}\right)} K\left(\frac{2 \sqrt{x x^{\prime}}}{x+x^{\prime}}\right) \tilde{k}_{-} H\left(\frac{\left(x^{\prime}-\tilde{x}_{-}\right) \sqrt[3]{\Omega_{-}}}{h^{2 / 3}}\right) d x^{\prime} \\
+ & h^{1 / 3} \int_{\tilde{x}_{-}+\varepsilon}^{\tilde{x}_{+}-\varepsilon} \frac{1}{\pi\left(x+x^{\prime}\right)} K\left(\frac{2 \sqrt{x x^{\prime}}}{x+x^{\prime}}\right) \frac{k d x^{\prime}}{S^{\prime}\left(x^{\prime}, h\right)} \\
+ & \Omega_{+} \int_{\tilde{x}_{+}-\varepsilon}^{\tilde{x}_{+}-h^{2 / 3} / \sqrt[3]{\Omega_{+}}} \frac{2 \tilde{x}_{+}}{\left(x+x^{\prime}\right)} K\left(\frac{2 \sqrt{x x^{\prime}}}{x+x^{\prime}}\right) \tilde{k}_{+} H\left(\left(\tilde{x}_{+}-x^{\prime}\right) \frac{\sqrt[3]{\Omega_{+}}}{h^{2 / 3}}\right) d x^{\prime} \\
+ & h^{2 / 3}\left\{\frac{4 \tilde{x}_{-}}{x+\tilde{x}_{-}} K\left(\frac{2 \sqrt{x \tilde{x}_{-}}}{x+\tilde{x}_{-}}\right) \Omega_{-}^{2 / 3} D_{-1}\left(\tilde{k}_{-}\right)\right. \\
+ & \left.\frac{4 \tilde{x}_{+}}{x+\tilde{x}_{+}} K\left(\frac{2 \sqrt{x \tilde{x}_{+}}}{x+\tilde{x}_{+}}\right) \Omega_{+}^{2 / 3} D_{-1}\left(\tilde{k}_{+}\right)\right\}+h^{8 / 9} \rho\left(\frac{k}{2 \pi x S^{\prime}}\right)^{2 / 3} \\
+ & \frac{h^{10 / 9} \sigma}{54 k^{2 / 3}}\left[\frac{\left(2 \pi \tilde{x}_{-}\right)^{2 / 3}\left(U^{\prime}\left(\tilde{x}_{-}\right)\right)^{4 / 3}}{\left(x-\tilde{x}_{-}\right)^{2 / 3}}+\frac{\left(2 \pi \tilde{x}_{+}\right)^{2 / 3}\left|U^{\prime}\left(\tilde{x}_{+}\right)\right|^{4 / 3}}{\left(\tilde{x}_{+}-x\right)^{2 / 3}}\right] \\
+ & 2 h^{4 / 3}\left[\frac{\sqrt[3]{\Omega_{-}} D_{1}\left(\tilde{k}_{-}\right)}{x-\tilde{x}_{-}}+\frac{\sqrt[3]{\Omega_{+}} D_{1}\left(\tilde{k}_{+}\right)}{\tilde{x}_{+}-x}\right] \\
& -\frac{h^{4 / 3} u}{243 k^{2}}\left\{\frac{\left(2 \pi \tilde{x}_{-}\right)^{2}\left(U^{\prime}\left(\tilde{x}_{-}\right)\right)^{3}}{x-\tilde{x}_{-}}+\frac{\left(2 \pi \tilde{x}_{+}\right)^{2}\left|U^{\prime}\left(\tilde{x}_{+}\right)\right|^{3}}{\tilde{x}_{+}-x}\right\}=R(x, h) .
\end{aligned}
$$

Здесь

$$
R(x, h)=O\left(h^{1+\frac{1}{57}} \ln \frac{1}{h}\right)+O\left(\frac{h^{13 / 9}}{\left(x-\tilde{x}_{-}\right)^{7 / 6}}\right)+O\left(\frac{h^{13 / 9}}{\left(\tilde{x}_{+}-x\right)^{7 / 6}}\right), \quad h \rightarrow 0 .
$$

Положим в $(5.7),(5.12)-(5.15),(5.23)$ остаточные члены равными нулю, а константы $\tilde{k}_{-}, \tilde{k}_{+}$зададим равенствами (4.22), (4.23). Кроме того, пусть $\varepsilon=h^{26 / 57}$. Таким образом, будет получена система уравнений для нахождения функций $S(x, h)$ при $x \in\left(\tilde{x}_{-}+\varepsilon, \tilde{x}_{+}-\varepsilon\right)$, а также констант $k, \tilde{x}_{-}, \tilde{x}_{+}, \Omega_{-}, \Omega_{+}$. Назовем эту систему задачей для фазы.

ЗАмЕчаниЕ 5.1. Константы $\Omega_{ \pm}$необходимы для того, чтобы определить $g_{ \pm}(x, y)$ вблизи точек $\tilde{x}_{ \pm}$. 


\section{§ 6. Правило квантования. Оценка невязки. Формулировка основной теоремы}

6.1. Правило квантования. Для построения глобальной асимптотики задачи (1.11)-(1.13) остается получить правило квантования - условие, обеспечиваюшее гладкое сшивание асимптотического решения. Именно из этого правила находятся асимптотические собственные значения (1.11)-(1.13).

В $\S 2,3$ мы построили ВКБ-приближение вида

$$
\begin{aligned}
g= & \frac{1}{h^{5 / 18}}\left\{\left[B_{0}+h^{2 / 9} B_{1}+h^{4 / 9} B_{2}+O\left(h^{5 / 9} B_{3}\right)\right] \cos \frac{S(x, h)}{h}\right. \\
& \left.+\left[h^{1 / 9} I_{0}+h^{3 / 9} I_{1}+O\left(h^{5 / 9} I_{2}\right)\right] \sin \frac{S(x, h)}{h}\right\} .
\end{aligned}
$$

Введем функцию

$$
\begin{aligned}
S_{0,0}(\xi, \tilde{k}) \stackrel{\text { def }}{=} & \frac{2}{3} \xi^{3 / 2}-A_{-1} \sqrt{\xi}(2 \ln \xi-4)+2 A_{0} \sqrt{\xi} \\
& +3 \rho \tilde{k}^{2 / 3} \xi^{1 / 6}-\tilde{k} \pi^{2} A_{-1} \ln \xi+\delta .
\end{aligned}
$$

Так как при $x-\tilde{x}_{-}$порядка $\varepsilon$ асимптотики $g$ и $g_{-}$согласованы, то, используя $(6.2)$, (4.19), (4.20), для функции $S$ из (6.1) имеем

$$
\frac{S(x, h)}{h}=S_{0,0}\left(\frac{\varepsilon \sqrt[3]{\Omega_{-}}}{h^{2 / 3}}, \tilde{k}_{-}\right)+\frac{1}{h} \int_{\tilde{x}_{-}+\varepsilon}^{x} S^{\prime}\left(x^{\prime}, h\right) d x^{\prime}+O\left(\frac{h^{1 / 9}}{\varepsilon^{1 / 6}}\right), \quad h \rightarrow 0 .
$$

При построении ВКБ-асимптотики мы для определенности считали, что $k>0$, $S^{\prime}>0, y=(2 \pi x)^{-1 / 6}\left(k / S^{\prime}\right)^{2 / 3}>0$. Совершенно аналогично рассматривается случай, когда $k<0, S^{\prime}<0, y=(2 \pi x)^{-1 / 6}\left(k / S^{\prime}\right)^{2 / 3}>0$. В результате замены $k$ на $-k, S^{\prime}$ на $-S^{\prime}$ функции $B_{0}, B_{1}, B_{2}$ в (6.1) не изменятся, а $I_{0}, I_{1}$ поменяют знак. Сама же задача для фазы останется прежней. Надо лишь формулы, задаюшие $\tilde{k}_{-}$, $\tilde{k}_{+}$, записать в виде $\tilde{k}_{-}=|k| /\left(2 \pi \tilde{x}_{-} \Omega_{-}^{4 / 3}\right), \tilde{k}_{+}=|k| /\left(2 \pi \tilde{x}_{+} \Omega_{+}^{4 / 3}\right)$. В результате получаем разложение

$$
\begin{aligned}
g^{*}= & \frac{1}{h^{5 / 18}}\left\{\left[B_{0}+h^{2 / 9} B_{1}+h^{4 / 9} B_{2}+O\left(h^{5 / 9} B_{3}\right)\right] \cos \frac{S(x, h)}{h}\right. \\
& \left.+\left[-h^{1 / 9} I_{0}-h^{3 / 9} I_{1}+O\left(h^{5 / 9} I_{2}\right)\right] \sin \frac{S(x, h)}{h}\right\},
\end{aligned}
$$

где

$$
\frac{S(x, h)}{h}=S_{0,0}\left(\frac{\varepsilon \sqrt[3]{\Omega_{+}}}{h^{2 / 3}}, \tilde{k}_{+}\right)+\frac{1}{h} \int_{x}^{\tilde{x}_{+}-\varepsilon} S^{\prime}\left(x^{\prime}, h\right) d x^{\prime}+O\left(\frac{h^{1 / 9}}{\varepsilon^{1 / 6}}\right), \quad h \rightarrow 0,
$$

которое при $\tilde{x}_{+}-x$ порядка $\varepsilon$ согласовано с заданной формулами (4.14), (4.15) асимптотикой $g_{+}$. 
Чтобы обеспечить сушествование глобального асимптотического решения (1.11)-(1.13), потребуем тождественного совпадения функций $g$ и $g^{*}$ при $x \in\left(\tilde{x}_{-}+\right.$ $\left.\varepsilon, \tilde{x}_{+}-\varepsilon\right)$. В силу формул $(6.1),(6.3)-(6.5)$ получаем равенства

$$
\begin{aligned}
& \cos \left(S_{0,0}\left(\frac{\varepsilon \sqrt[3]{\Omega_{-}}}{h^{2 / 3}}, \tilde{k}_{-}\right)+\frac{1}{h} \int_{\tilde{x}_{-}+\varepsilon}^{x} S^{\prime}\left(x^{\prime}, h\right) d x^{\prime}+O\left(\frac{h^{1 / 9}}{\varepsilon^{1 / 6}}\right)\right) \\
& \equiv \cos \left(S_{0,0}\left(\frac{\varepsilon \sqrt[3]{\Omega_{+}}}{h^{2 / 3}}, \tilde{k}_{+}\right)+\frac{1}{h} \int_{x}^{\tilde{x}_{+}-\varepsilon} S^{\prime}\left(x^{\prime}, h\right) d x^{\prime}+O\left(\frac{h^{1 / 9}}{\varepsilon^{1 / 6}}\right)\right) \\
& \sin \left(S_{0,0}\left(\frac{\varepsilon \sqrt[3]{\Omega_{-}}}{h^{2 / 3}}, \tilde{k}_{-}\right)+\frac{1}{h} \int_{\tilde{x}_{-}+\varepsilon}^{x} S^{\prime}\left(x^{\prime}, h\right) d x^{\prime}+O\left(\frac{h^{1 / 9}}{\varepsilon^{1 / 6}}\right)\right) \\
& \equiv-\sin \left(S_{0,0}\left(\frac{\varepsilon \sqrt[3]{\Omega_{+}}}{h^{2 / 3}}, \tilde{k}_{+}\right)+\frac{1}{h} \int_{x}^{\tilde{x}_{+}-\varepsilon} S^{\prime}\left(x^{\prime}, h\right) d x^{\prime}+O\left(\frac{h^{1 / 9}}{\varepsilon^{1 / 6}}\right)\right) .
\end{aligned}
$$

Справедлива

Лемма 6.1. Равенства (6.6) имеют место тогда и только тогда, когда выполнено условие

$$
\begin{gathered}
S_{0,0}\left(\frac{\varepsilon \sqrt[3]{\Omega_{-}}}{h^{2 / 3}}, \tilde{k}_{-}\right)+\frac{1}{h} \int_{\tilde{x}_{-}+\varepsilon}^{\tilde{x}_{+}-\varepsilon} S^{\prime}\left(x^{\prime}, h\right) d x^{\prime}+S_{0,0}\left(\frac{\varepsilon \sqrt[3]{\Omega_{+}}}{h^{2 / 3}}, \tilde{k}_{+}\right) \\
=2 \pi n+O\left(h^{1 / 9} / \varepsilon^{1 / 6}\right)
\end{gathered}
$$

где $n-$ челье.

Наряду с $g$ и $g^{*}$ рассмотрим также решение $-g^{*}$, которое получается из $g$ заменой $k$ на $-k, S^{\prime}$ на $-S^{\prime}, y=(2 \pi x)^{-1 / 6}\left(k / S^{\prime}\right)^{2 / 3}$ на $-y$. Возможность такой замены следует из формул (3.11). Потребовав при $x \in\left(\tilde{x}_{-}+\varepsilon, \tilde{x}_{+}-\varepsilon\right)$ тождественного совпадения $g$ и $-g^{*}$, приходим к условию

$$
\begin{gathered}
S_{0,0}\left(\frac{\varepsilon \sqrt[3]{\Omega_{-}}}{h^{2 / 3}}, \tilde{k}_{-}\right)+\frac{1}{h} \int_{\tilde{x}_{-}+\varepsilon}^{\tilde{x}_{+}-\varepsilon} S^{\prime}\left(x^{\prime}, h\right) d x^{\prime}+S_{0,0}\left(\frac{\varepsilon \sqrt[3]{\Omega_{+}}}{h^{2 / 3}}, \tilde{k}_{+}\right) \\
=\pi+2 \pi n+O\left(h^{1 / 9} / \varepsilon^{1 / 6}\right)
\end{gathered}
$$

где $n$ - целые. Наконец, объединяя (6.7), (6.8), получаем следующее правило квантования типа Бора-Зоммерфельда:

$$
S_{0,0}\left(\frac{\varepsilon \sqrt[3]{\Omega_{-}}}{h^{2 / 3}}, \tilde{k}_{-}\right)+\frac{1}{h} \int_{\tilde{x}_{-}+\varepsilon}^{\tilde{x}_{+}-\varepsilon} S^{\prime}\left(x^{\prime}, h\right) d x^{\prime}+S_{0,0}\left(\frac{\varepsilon \sqrt[3]{\Omega_{+}}}{h^{2 / 3}}, \tilde{k}_{+}\right)=\pi n .
$$

Здесь $n$ - целые; $S^{\prime}(x, h)>0$, а также $k>0$ и $\tilde{x}_{-}, \tilde{x}_{+}, \Omega_{-}, \Omega_{+}$являются решением задачи для фазы; $\varepsilon=h^{26 / 57}, \tilde{k}_{-}, \tilde{k}_{+}$определены формулами (4.22), (4.23), а $S_{0,0}(\xi, \tilde{k})$ - формулой $(6.2)$.

Правило (6.9) дает алгебраическое уравнение для определения $\lambda=\lambda_{n}(h)$. В (6.9) входят параметры $n \rightarrow \infty$ и $h \rightarrow 0$. Пусть их произведение ограничено некоторыми константами $C_{1}, C_{2}$ так, что

$$
0<C_{1} \leqslant h n \leqslant C_{2}<\infty \text {. }
$$

Тогда $\lambda_{n}(h)=O(1)$ при $n \rightarrow \infty$. 
ЗАмЕчАниЕ 6.1. При выводе (6.9) мы пренебрегли слагаемым $O\left(h^{1 / 9} / \varepsilon^{1 / 6}\right)$. Кроме того, имеется еше погрешность при нахождении $S^{\prime}$ (см. (5.24)). Поэтому правило квантования (6.9) получено с точностью $O\left(h^{1 / 57} \ln \frac{1}{h}\right), h \rightarrow 0$.

6.2. Оценка невязки. Итак, глобальные асимптотические решения $g=g_{n}$ задачи (1.11)-(1.13) построены. Пусть $\lambda=\lambda_{n}(h)$ подчинены правилу (6.9). Тогда при $x \in\left(\tilde{x}_{-}+\varepsilon, \tilde{x}_{+}-\varepsilon\right)$ асимптотические решения $g_{n}=g_{n}^{\text {ВКБ }}$ задаются формулами (2.24), (3.1), (3.2), где остаточные члены таковы, что выполнено (2.27). Для $x$, находяшихся вблизи и левее $\tilde{x}_{-}$, функции $g_{n}$ определяются равенствами $(4.1),(4.2)$, а для $x$, расположенных вблизи и правее $\tilde{x}_{+},-$равенствами $(4.14),(4.15)$. Входящие в $(4.1),(4.14)$ функции $G_{-}, G_{+}$удовлетворяют модельному уравнению (1.4). Вблизи от точек $\tilde{x}_{-}+\varepsilon, \tilde{x}_{+}-\varepsilon$ асимптотики согласованы между собой. Используя разбиение единицы, $g_{n}$ можно записать в виде

$$
g_{n}=\chi_{-}^{*} g_{-}+\left(1-\chi_{-}^{*}-\chi_{+}^{*}\right) g_{n}^{\mathrm{BKБ}}+\chi_{+}^{*} g_{+} .
$$

Подставим $g=g_{n}$ в уравнение $(1.11)$ и оценим в норме $L^{2}\left(\mathbb{R}_{+} \times \mathbb{R}\right)$ возникающую невязку $R_{n}$. Так как $G_{ \pm}(\xi, \eta)$ при $\xi \rightarrow-\infty$ экспоненциально убывают, то

$$
\int_{0}^{\tilde{x}_{-}-\varepsilon} \int_{-\infty}^{\infty} R_{n}^{2} d y d x=O\left(h^{\infty}\right), \quad \int_{\tilde{x}_{+}+\varepsilon}^{\infty} \int_{-\infty}^{\infty} R_{n}^{2} d y d x=O\left(h^{\infty}\right), \quad h \rightarrow 0 .
$$

В силу $(2.16),(5.23)$ при $x \in\left(\tilde{x}_{-}+\varepsilon, \tilde{x}_{+}-\varepsilon\right)$ получаем $R_{n}=O\left(r^{*}\right)+O(R T)$, где $r^{*}, R$ определяются равенствами $(2.15),(2.17),(5.24)$. Наконец, если выполнено неравенство $\left|\tilde{x}_{-}-x\right|<\varepsilon$, то $R_{n}=r_{-}$, а если $\left|\tilde{x}_{+}-x\right|<\varepsilon$, то $R_{n}=r_{+}$. Здесь $r_{-}$, $r_{+}$заданы формулами (4.13), (4.17), в которых $g=g_{n}$.

Справедлива следующая оценка невязки $R_{n}$ :

$$
\left\|R_{n}\right\|_{L^{2}\left(\mathbb{R}_{+} \times \mathbb{R}\right)}=O\left(h^{1+1 / 57} \ln \frac{1}{h}\right), \quad h \rightarrow 0 .
$$

Кроме того, $g_{n}$ удовлетворяет условию нормировки (1.12) с точностью $O\left(h^{\frac{2}{3}+\frac{1}{57}}\right)$, а также условию (1.13) с точностью $O(h)$.

Итак, доказана основная теорема.

ТЕОРема 6.1. Пусть параметры $h$ и $n$ удовлетворяют условию (6.10). Тогда числа $\lambda=\lambda_{n}(h)$, заданные правилом квантования (6.9), являются асимптотическими собственными значениями задачи (1.11)-(1.13) с точностью $O\left(n^{-1-1 / 57} \ln n\right)$ при $n \rightarrow \infty$. Соответствующие асимптотические собственнье функиии $g=g_{n}$ удовлетворяют $(1.11)$ с точностью $O\left(n^{-1-1 / 57} \ln n\right)$ в норме $L^{2}\left(\mathbb{R}_{+} \times \mathbb{R}\right)$, условию нормировки $(1.12)$ с точностью $O\left(n^{-2 / 3-1 / 57}\right)$, а также условию (1.13) с точностью $O\left(n^{-1}\right)$.

ЗАмЕчАнИЕ 6.2. Отметим, что построенные асимптотические решения $g_{n}(x, y)$ в конфигурационном пространстве $\mathbb{R}_{+} \times \mathbb{R}$ при $n \rightarrow \infty$ в слабом смысле (как обобщенные функции) сосредоточены на маломерном подмногообразии, а именно на отрезке $\left[\tilde{x}_{-}, \tilde{x}_{+}\right]$прямой $y=0$. В фазовом пространстве, т.е. в кокасательном расслоении на $\mathbb{R}_{+} \times \mathbb{R}$, фронтом осцилляций этих функций служит изотропное подмногообразие - плоский диск $D(1.3)$. 


\section{§ 7. Главное приближение к фазе}

В этом параграфе мы построим асимптотическое решение задачи для главного приближения к фазе. Эта задача возникает при нахождении главного приближения для асимптотических собственных значений, когда в задаче для фазы можно отбросить ряд высших квантовых поправок. Из этой задачи находится главное приближение к фазе $S$, а также приближенные значения констант $k, \tilde{x}_{-}, \tilde{x}_{+}$.

7.1. Задача для главного приближения к фазе. Рассмотрим уравнения $(5.7),(5.12)-(5.15),(5.23)$ (задача для фозы). Из (5.14), (5.15) вытекает, что

$$
\Omega_{-}=U^{\prime}\left(\tilde{x}_{-}\right)+O\left(h^{1 / 3}\right), \quad \Omega_{+}=-U^{\prime}\left(\tilde{x}_{+}\right)+O\left(h^{1 / 3}\right), \quad h \rightarrow 0 .
$$

Поскольку для заданной (5.1) функции $H(\xi)$ при $\tau \rightarrow+\infty$ справедливы равенства

$$
\int_{1}^{\tau}(\ln \xi) H(\xi) d \xi=2 \sqrt{\tau}(\ln \tau-2)+O(1), \quad \int_{1}^{\tau} H(\xi) d \xi=2 \sqrt{\tau}+O(1)
$$

то из (5.12), (5.13) получаем

$$
\begin{aligned}
& U\left(\tilde{x}_{-}\right)+2 h^{1 / 3}\left\{-h^{1 / 3} \Omega_{-}^{2 / 3} \tilde{k}_{-} \sqrt{e_{-}}\left(\ln \left(\frac{\varepsilon}{8 \tilde{x}_{-}}\right)-2\right)\right. \\
& +\int_{\tilde{x}_{-}+\varepsilon}^{\tilde{x}_{+}-\varepsilon} \frac{1}{\pi\left(\tilde{x}_{-}+x^{\prime}\right)} K\left(\frac{2 \sqrt{\tilde{x}_{-} x^{\prime}}}{\tilde{x}_{-}+x^{\prime}}\right) \frac{k d x^{\prime}}{2 S^{\prime}\left(x^{\prime}, h\right)} \\
& \left.+h^{1 / 3} \frac{2 \tilde{x}_{+}}{\tilde{x}_{+}+\tilde{x}_{-}} K\left(\frac{2 \sqrt{\tilde{x}_{+} \tilde{x}_{-}}}{\tilde{x}_{+}+\tilde{x}_{-}}\right) \Omega_{+}^{2 / 3} \tilde{k}_{+} \sqrt{e_{+}}\right\}=O\left(h^{2 / 3} \ln \frac{1}{h}\right) \\
& U\left(\tilde{x}_{+}\right)+2 h^{1 / 3}\left\{h^{1 / 3} \frac{2 \tilde{x}_{-}}{\tilde{x}_{+}+\tilde{x}_{-}} K\left(\frac{2 \sqrt{\tilde{x}_{-} \tilde{x}_{+}}}{\tilde{x}_{-}+\tilde{x}_{+}}\right) \Omega_{-}^{2 / 3} \tilde{k}_{-} \sqrt{e_{-}}\right. \\
& +\int_{\tilde{x}_{-}+\varepsilon}^{\tilde{x}_{+}-\varepsilon} \frac{1}{\pi\left(\tilde{x}_{+}+x^{\prime}\right)} K\left(\frac{2 \sqrt{\tilde{x}_{+} x^{\prime}}}{\tilde{x}_{+}+x^{\prime}}\right) \frac{k d x^{\prime}}{2 S^{\prime}\left(x^{\prime}, h\right)} \\
& \left.-h^{1 / 3} \Omega_{+}^{2 / 3} \tilde{k}_{+\sqrt{e_{+}}}\left(\ln \left(\frac{\varepsilon}{8 \tilde{x}_{+}}\right)-2\right)\right\}=O\left(h^{2 / 3} \ln \frac{1}{h}\right) .
\end{aligned}
$$

Здесь $e_{ \pm}, \tilde{k}_{ \pm}$определены формулами (5.5), (4.22), (4.23), $\varepsilon=h^{26 / 57}, h \rightarrow 0$.

Далее, так как в силу (5.1), (5.2)

$$
D_{-1}\left(\tilde{k}_{ \pm}\right)+\int_{1}^{e_{ \pm}} \frac{\tilde{k}_{ \pm}}{2} H(\xi) d \xi=A_{-1}\left(\tilde{k}_{ \pm}\right)+\tilde{k}_{ \pm} \sqrt{e_{ \pm}}+O\left(\frac{h^{1 / 3}}{\sqrt{\varepsilon}} \ln \frac{1}{h}\right)
$$

то условие нормировки (5.7) принимает вид

$$
\begin{aligned}
& h^{1 / 3} \Omega_{-}^{2 / 3} 2 \pi \tilde{x}_{-}\left[A_{-1}\left(\tilde{k}_{-}\right)+\tilde{k}_{-} \sqrt{e_{-}}\right]+\int_{\tilde{x}_{-}+\varepsilon}^{\tilde{x}_{+}-\varepsilon} \frac{k d x^{\prime}}{2 S^{\prime}\left(x^{\prime}, h\right)} \\
& \quad+h^{1 / 3} \Omega_{+}^{2 / 3} 2 \pi \tilde{x}_{+}\left[A_{-1}\left(\tilde{k}_{+}\right)+\tilde{k}_{+} \sqrt{e_{+}}\right]=1+O\left(h^{1 / 3+2 / 19} \ln \frac{1}{h}\right), \quad h \rightarrow 0
\end{aligned}
$$

Рассмотрим, наконец, уравнение (5.23). 
Лемма 7.1. При $h \rightarrow 0, x \in\left(\tilde{x}_{-}+\varepsilon, \tilde{x}_{+}-\varepsilon\right)$ имеют место равенства

$$
\begin{aligned}
& \Omega_{-} \int_{\tilde{x}_{-}+h^{2 / 3} / \sqrt[3]{\Omega_{-}}}^{\tilde{x}_{-}+\varepsilon} \frac{2 \tilde{x}_{-}}{\left(x+x^{\prime}\right)} K\left(\frac{2 \sqrt{x x^{\prime}}}{x+x^{\prime}}\right) \tilde{k}_{-} H\left(\frac{\left(x^{\prime}-\tilde{x}_{-}\right) \sqrt[3]{\Omega_{-}}}{h^{2 / 3}}\right) d x^{\prime} \\
& +h^{2 / 3} \frac{4 \tilde{x}_{-}}{x+\tilde{x}_{-}} K\left(\frac{2 \sqrt{x \tilde{x}_{-}}}{x+\tilde{x}_{-}}\right) \Omega_{-}^{2 / 3} D_{-1}\left(\tilde{k}_{-}\right) \\
& =\frac{h^{2 / 3} \Omega_{-}^{2 / 3} 4 \tilde{x}_{-}}{x+\tilde{x}_{-}}\left\{K\left(\frac{2 \sqrt{x \tilde{x}_{-}}}{x+\tilde{x}_{-}}\right)\left[A_{-1}\left(\tilde{k}_{-}\right)+\tilde{k}_{-\sqrt{e_{-}}}\right]\right. \\
& \left.-\frac{\tilde{k}_{-}}{2} \int_{0}^{e_{-}} \ln \left|1-\frac{\xi^{\prime} h^{2 / 3}}{\left(x-\tilde{x}_{-}\right) \sqrt[3]{\Omega_{-}}}\right| \frac{d \xi^{\prime}}{\sqrt{\xi^{\prime}}}\right\} \\
& +O\left(h^{\frac{2}{3}+\frac{2}{19}} \ln \frac{1}{h}\left(1+|\ln | x-\tilde{x}_{-}||\right)\right), \\
& \Omega_{+} \int_{\tilde{x}_{+}-\varepsilon}^{\tilde{x}_{+}-h^{2 / 3} / \sqrt[3]{\Omega_{+}}} \frac{2 \tilde{x}_{+}}{\left(x+x^{\prime}\right)} K\left(\frac{2 \sqrt{x x^{\prime}}}{x+x^{\prime}}\right) \tilde{k}_{+} H\left(\frac{\left(\tilde{x}_{+}-x^{\prime}\right) \sqrt[3]{\Omega_{+}}}{h^{2 / 3}}\right) d x^{\prime} \\
& +\frac{h^{2 / 3} 4 \tilde{x}_{+}}{x+\tilde{x}_{+}} K\left(\frac{2 \sqrt{x \tilde{x}_{+}}}{x+\tilde{x}_{+}}\right) \Omega_{+}^{2 / 3} D_{-1}\left(\tilde{k}_{+}\right) \\
& =\frac{h^{2 / 3} \Omega_{+}^{2 / 3} 4 \tilde{x}_{+}}{x+\tilde{x}_{+}}\left\{K\left(\frac{2 \sqrt{x \tilde{x}_{+}}}{x+\tilde{x}_{+}}\right)\left[A_{-1}\left(\tilde{k}_{+}\right)+\tilde{k}_{+} \sqrt{e_{+}}\right]\right. \\
& \left.-\frac{\tilde{k}_{+}}{2} \int_{0}^{e_{+}} \ln \left|1-\frac{\xi^{\prime} h^{2 / 3}}{\left(\tilde{x}_{+}-x\right) \sqrt[3]{\Omega_{+}}}\right| \frac{d \xi^{\prime}}{\sqrt{\xi^{\prime}}}\right\} \\
& +O\left(h^{\frac{2}{3}+\frac{2}{19}} \ln \frac{1}{h}\left(1+|\ln | x-\tilde{x}_{+}||\right)\right), \\
& h^{8 / 9} \rho \frac{k^{2 / 3}}{\left(2 \pi x S^{\prime}\right)^{2 / 3}}=O\left(\frac{h^{8 / 9}}{\left(x-\tilde{x}_{-}\right)^{1 / 3}}\right)+O\left(\frac{h^{8 / 9}}{\left(\tilde{x}_{+}-x\right)^{1 / 3}}\right) \text {. }
\end{aligned}
$$

ДокАЗАТЕЛЬСТво. Воспользовавшись (5.16), (7.4), а также соотношением

$$
\begin{aligned}
& \Omega_{-} \int_{\tilde{x}_{-}+h^{2 / 3} / \sqrt[3]{\Omega_{-}}}^{\tilde{x}_{-}+\varepsilon} \ln \left|x-x^{\prime}\right| \tilde{k}_{-} H\left(\frac{\left(x^{\prime}-\tilde{x}_{-}\right) \sqrt[3]{\Omega_{-}}}{h^{2 / 3}}\right) d x^{\prime} \\
& +2 h^{2 / 3} \Omega_{-}^{2 / 3} D_{-1}\left(\tilde{k}_{-}\right) \ln \left|x-\tilde{x}_{-}\right| \\
& =2 h^{2 / 3} \Omega_{-}^{2 / 3}\left\{\ln \left|x-\tilde{x}_{-}\right|\left[A_{-1}\left(\tilde{k}_{-}\right)+\sqrt{e_{-}} \tilde{k}_{-}\right]\right. \\
& \left.+\frac{\tilde{k}_{-}}{2} \int_{0}^{e_{-}} \ln \left|1-\frac{\xi^{\prime} h^{2 / 3}}{\left(x-\tilde{x}_{-}\right) \sqrt[3]{\Omega_{-}}}\right| \frac{d \xi^{\prime}}{\sqrt{\xi^{\prime}}}\right\} \\
& +O\left(h^{2 / 3+2 / 19} \ln \frac{1}{h}\left(1+|\ln | x-\tilde{x}_{-}||\right)\right),
\end{aligned}
$$

приходим к (7.6). Аналогично проверяется (7.7). Так как при $x \rightarrow \tilde{x}_{ \pm}$имеем $S^{\prime}(x, h) \sim \sqrt{U^{\prime}\left(x_{ \pm}\right)\left(x-\tilde{x}_{ \pm}\right)}$, то оценка $(7.8)$ также справедлива. Лемма доказана. 
В силу (7.6)-(7.8) уравнение (5.23) принимает вид

$$
\begin{aligned}
-\left(S^{\prime}\right)^{2} & +U(x)+h^{1 / 3} \int_{\tilde{x}_{-}+\varepsilon}^{\tilde{x}_{+}-\varepsilon} \frac{1}{\pi\left(x+x^{\prime}\right)} K\left(\frac{2 \sqrt{x x^{\prime}}}{x+x^{\prime}}\right) \frac{k d x^{\prime}}{S^{\prime}\left(x^{\prime}, h\right)} \\
& +\frac{h^{2 / 3} \Omega_{-}^{2 / 3} 4 \tilde{x}_{-}}{x+\tilde{x}_{-}}\left\{K\left(\frac{2 \sqrt{x \tilde{x}_{-}}}{x+\tilde{x}_{-}}\right)\left[A_{-1}\left(\tilde{k}_{-}\right)+\tilde{k}_{-} \sqrt{e_{-}}\right]\right. \\
& \left.-\frac{\tilde{k}_{-}}{2} \int_{0}^{e_{-}} \ln \left|1-\frac{\xi^{\prime} h^{2 / 3}}{\left(x-\tilde{x}_{-}\right) \sqrt[3]{\Omega_{-}}}\right| \frac{d \xi^{\prime}}{\sqrt{\xi^{\prime}}}\right\} \\
& +\frac{h^{2 / 3} \Omega_{+}^{2 / 3} 4 \tilde{x}_{+}}{x+\tilde{x}_{+}}\left\{K\left(\frac{2 \sqrt{x \tilde{x}_{+}}}{x+\tilde{x}_{+}}\right)\left[A_{-1}\left(\tilde{k}_{+}\right)+\tilde{k}_{+} \sqrt{e_{+}}\right]\right. \\
& \left.-\frac{\tilde{k}_{+}}{2} \int_{0}^{e_{+}} \ln \left|1-\frac{\xi^{\prime} h^{2 / 3}}{\left(\tilde{x}_{+}-x\right) \sqrt[3]{\Omega_{+}}}\right| \frac{d \xi^{\prime}}{\sqrt{\xi^{\prime}}}\right\}=R^{*}
\end{aligned}
$$

где

$$
\begin{aligned}
& R^{*}=O\left(h^{\frac{2}{3}+\frac{2}{19}} \ln \frac{1}{h}\left(1+|\ln | x-\tilde{x}_{-}||+|\ln | x-\tilde{x}_{+}||\right)\right) \\
& +O\left(\frac{h^{8 / 9}}{\left(x-\tilde{x}_{-}\right)^{1 / 3}}\right)+O\left(\frac{h^{8 / 9}}{\left(\tilde{x}_{+}-x\right)^{1 / 3}}\right) .
\end{aligned}
$$

Здесь $e_{ \pm}, \tilde{k}_{ \pm}$определены (5.5), (4.22), (4.23), $\varepsilon=h^{26 / 57}, x \in\left(\tilde{x}_{-}+\varepsilon, \tilde{x}_{+}-\varepsilon\right)$.

Таким образом, получена задача для главного приближения к фазе. Она состоит из уравнений $(7.2),(7.3),(7.5),(7.9)$, где $\Omega_{ \pm}$задаются соотношениями $(7.1)$.

7.2. Нахождение главного приближения к фазе. Перейдем к построению решения задачи для главного приближения к фазе. Точность, с которой строится решение, соответствует точности, с которой получены уравнения (7.2), $(7.3),(7.5),(7.9)$.

Но прежде всего введем ряд функций, необходимых для построения разложений, и изучим свойства этих функций. Среди них имеются негладкие (заданная формулой (1.17) функция $\Omega(x)$ не дифференцируема при $\left.x=\tilde{x}_{ \pm}\right)$, что приводит к весьма нестандартным асимптотикам.

ЛЕмма 7.2. Справедливы равенства

$$
\begin{aligned}
& \Omega(x)=\Omega\left(x_{-}\right)-\frac{\sqrt{x_{-}-x}}{x_{-} \sqrt{U^{\prime}\left(x_{-}\right)}} \theta\left(x_{-}-x\right)+\left(x-x_{-}\right) \Phi_{-} \\
& +\left(-\frac{U^{\prime \prime}\left(x_{-}\right)}{12 x_{-}\left(U^{\prime}\left(x_{-}\right)\right)^{3 / 2}}+\frac{1}{3 x_{-}^{2} \sqrt{U^{\prime}\left(x_{-}\right)}}\right)\left(x_{-}-x\right)^{3 / 2} \theta\left(x_{-}-x\right) \\
& +O\left(\left(x-x_{-}\right)^{2}\right), \quad x \rightarrow x_{-}, \\
& \Omega(x)=\Omega\left(x_{+}\right)-\frac{\sqrt{x-x_{+}}}{x_{+} \sqrt{-U^{\prime}\left(x_{+}\right)}} \theta\left(x-x_{+}\right)+\left(x_{+}-x\right) \Phi_{+}
\end{aligned}
$$




$$
\begin{aligned}
& +\left(-\frac{U^{\prime \prime}\left(x_{+}\right)}{12 x_{+}\left(-U^{\prime}\left(x_{+}\right)\right)^{3 / 2}}+\frac{1}{3 x_{+}^{2} \sqrt{-U^{\prime}\left(x_{+}\right)}}\right)\left(x-x_{+}\right)^{3 / 2} \theta\left(x-x_{+}\right) \\
& +O\left(\left(x_{+}-x\right)^{2}\right), \quad x \rightarrow x_{+}
\end{aligned}
$$

əде

$$
\begin{aligned}
& \Phi_{-}=\frac{1}{2 \pi x_{-}}\left[\Phi_{-}^{*}-\int_{x_{-}}^{x_{+}} \frac{d x^{\prime}}{\left(x^{\prime}+x_{-}\right) \sqrt{U\left(x^{\prime}\right)}}\right]+\frac{1}{2} \int_{x_{-}}^{x_{+}} F_{-}\left(x^{\prime}, x_{-}\right) \frac{d x^{\prime}}{\sqrt{U\left(x^{\prime}\right)}} \\
& \Phi_{+}=\frac{1}{2 \pi x_{+}}\left[\Phi_{+}^{*}-\int_{x_{-}}^{x_{+}} \frac{d x^{\prime}}{\left(x^{\prime}+x_{+}\right) \sqrt{U\left(x^{\prime}\right)}}\right]+\frac{1}{2} \int_{x_{-}}^{x_{+}} F_{+}\left(x^{\prime}, x_{+}\right) \frac{d x^{\prime}}{\sqrt{U\left(x^{\prime}\right)}} .
\end{aligned}
$$

Здесь

$$
\begin{gathered}
\Phi_{-}^{*}=\int_{x_{-}}^{\infty} \frac{1}{\left(x^{\prime}-x_{-}\right)}\left(\frac{\theta\left(x_{+}-x^{\prime}\right)}{\sqrt{U\left(x^{\prime}\right)}}-\frac{1}{\sqrt{U^{\prime}\left(x_{-}\right)\left(x^{\prime}-x_{-}\right)}}\right) d x^{\prime}, \\
\Phi_{+}^{*}=\int_{-\infty}^{x_{+}} \frac{1}{\left(x_{+}-x^{\prime}\right)}\left(\frac{\theta\left(x^{\prime}-x_{-}\right)}{\sqrt{U\left(x^{\prime}\right)}}-\frac{1}{\sqrt{U^{\prime}\left(x_{+}\right)\left(x^{\prime}-x_{+}\right)}}\right) d x^{\prime}, \\
F_{+}\left(x^{\prime}, x_{-}\right)=\frac{2}{\pi}\left[\frac{1}{x_{+}-x^{\prime}}\left(\frac{1}{2 x_{+}} E\left(\frac{2 \sqrt{x_{+} x^{\prime}}}{x_{+}+x^{\prime}}\right)-\frac{1}{x_{+}+x^{\prime}}\right)\right. \\
\left.+\frac{1}{2 x_{+}\left(x_{+}+x^{\prime}\right)} K\left(\frac{2 \sqrt{x_{+} x^{\prime}}}{x_{+}+x^{\prime}}\right)\right],
\end{gathered}
$$

а функиии $\theta, F_{-}$задаются (4.10), (4.5).

ДокАЗАТЕЛЬСТво. В силу (4.7)

$$
\begin{aligned}
\Omega(x)= & \Omega\left(x_{-}\right)-\frac{1}{2 \pi x_{-}} \int_{x_{-}}^{x_{+}} \ln \left|\frac{x-x^{\prime}}{x_{-}-x^{\prime}}\right| \frac{d x^{\prime}}{\sqrt{U\left(x^{\prime}\right)}} \\
& +\frac{\left(x-x_{-}\right)}{2} \int_{x_{-}}^{x_{+}} F_{-}\left(x^{\prime}, x_{-}\right) \frac{d x^{\prime}}{\sqrt{U\left(x^{\prime}\right)}} \\
& +\int_{x_{-}}^{x_{+}} \frac{\left(x^{\prime}-x_{-}\right)}{2 \pi x_{-}\left(x_{-}+x^{\prime}\right)} \ln \left|\frac{x-x^{\prime}}{x_{-}-x^{\prime}}\right| \frac{d x^{\prime}}{\sqrt{U\left(x^{\prime}\right)}} \\
& +\left(x-x_{-}\right) \int_{x_{-}}^{x_{+}} \frac{1}{\pi\left(x_{-}+x^{\prime}\right)^{2}} \ln \left|\frac{x-x^{\prime}}{x_{-}-x^{\prime}}\right| \frac{d x^{\prime}}{\sqrt{U\left(x^{\prime}\right)}} \\
& +O\left(\left(x-x_{-}\right)^{2}\right), \quad x \rightarrow x_{-} .
\end{aligned}
$$

Изучим входящие в (7.13) интегралы. Так как

$$
\begin{gathered}
\int_{x_{-}}^{x_{+}} \ln \left|\frac{x-x^{\prime}}{x_{-}-x^{\prime}}\right| \frac{d x^{\prime}}{\sqrt{U\left(x^{\prime}\right)}}=\frac{2 \pi \sqrt{x_{-}-x}}{\sqrt{U^{\prime}\left(x_{-}\right)}} \theta\left(x_{-}-x\right)-\left(x-x_{-}\right) \Phi_{-}^{*} \\
+\frac{\pi}{6} \frac{U^{\prime \prime}\left(x_{-}\right)}{\left(U^{\prime}\left(x_{-}\right)\right)^{3 / 2}}\left(x_{-}-x\right)^{3 / 2} \theta\left(x_{-}-x\right)+O\left(\left(x-x_{-}\right)^{2}\right), \quad x \rightarrow x_{-},
\end{gathered}
$$


то имеем

$$
\begin{aligned}
& \int_{x_{-}}^{x_{+}} \frac{1}{\pi\left(x_{-}+x^{\prime}\right)^{2}} \ln \left|\frac{x-x^{\prime}}{x_{-}-x^{\prime}}\right| \frac{d x^{\prime}}{\sqrt{U\left(x^{\prime}\right)}} \\
& \quad=\frac{\sqrt{x_{-}-x}}{2 x_{-}^{2} \sqrt{U^{\prime}\left(x_{-}\right)}} \theta\left(x_{-}-x\right)+O\left(x_{-}-x\right), \quad x \rightarrow x_{-} .
\end{aligned}
$$

Кроме того, поскольку

$$
\begin{gathered}
\int_{0}^{\xi} \ln \left|1-\frac{1}{\tau}\right| \sqrt{\tau} d \tau=\left.\left\{\frac{2}{3} \tau^{3 / 2} \ln \left|1-\frac{1}{\tau}\right|-\frac{4}{3} \sqrt{\tau}+\frac{2}{3} \ln \left|\frac{1+\sqrt{\tau}}{1-\sqrt{\tau}}\right|\right\}\right|_{0} ^{\xi} \\
=-2 \sqrt{\xi}+O(1 / \sqrt{\xi}), \quad \xi \rightarrow+\infty, \\
\int_{0}^{\xi} \ln \left|1+\frac{1}{\tau}\right| \sqrt{\tau} d \tau=\left.\left\{\frac{2}{3} \tau^{3 / 2} \ln \left|1+\frac{1}{\tau}\right|+\frac{4}{3} \sqrt{\tau}-\frac{4}{3} \operatorname{arctg} \sqrt{\tau}\right\}\right|_{0} ^{\xi} \\
=2 \sqrt{\xi}-2 \pi / 3+O(1 / \sqrt{\xi}), \quad \xi \rightarrow+\infty,
\end{gathered}
$$

получаем

$$
\begin{aligned}
\int_{x_{-}}^{x_{+}} & \frac{\left(x^{\prime}-x_{-}\right)}{2 \pi x_{-}\left(x_{-}+x^{\prime}\right)} \ln \left|\frac{x-x^{\prime}}{x_{-}-x^{\prime}}\right| \frac{d x^{\prime}}{\sqrt{U\left(x^{\prime}\right)}} \\
= & -\frac{\left(x-x_{-}\right)}{2 \pi x_{-}} \int_{x_{-}}^{x_{+}} \frac{d x^{\prime}}{\left(x_{-}+x^{\prime}\right) \sqrt{U\left(x^{\prime}\right)}}-\frac{1}{6 x_{-}^{2} \sqrt{U^{\prime}\left(x_{-}\right)}}\left(x_{-}-x\right)^{3 / 2} \theta\left(x_{-}-x\right) \\
& +O\left(\left(x-x_{-}\right)^{2}\right), \quad x \rightarrow x_{-} .
\end{aligned}
$$

Равенство (7.11) вытекает из (7.13)-(7.16). Соотношение (7.12) проверяется аналогично. Лемма доказана.

Определим

$$
\begin{aligned}
& W_{0}(x, h) \stackrel{\text { def }}{=} U(x)+h^{1 / 3} k_{0} \Omega(x) \\
& \quad+h^{1 / 3} k_{0}\left\{\frac{\sqrt{x_{-}-x}}{x_{-} \sqrt{U^{\prime}\left(x_{-}\right)}} \theta\left(x_{-}-x\right)+\frac{\sqrt{x-x_{+}}}{x_{+} \sqrt{-U^{\prime}\left(x_{+}\right)}} \theta\left(x-x_{+}\right)\right\} \\
& \quad+h^{1 / 3} k_{0}\left\{\frac{U^{\prime \prime}\left(x_{-}\right)}{12 x_{-}\left(U^{\prime}\left(x_{-}\right)\right)^{3 / 2}}-\frac{1}{3 x_{-}^{2} \sqrt{U^{\prime}\left(x_{-}\right)}}\right)\left(x_{-}-x\right)^{3 / 2} \theta\left(x_{-}-x\right) \\
& \left.\quad+\left(\frac{U^{\prime \prime}\left(x_{+}\right)}{12 x_{+}\left(-U^{\prime}\left(x_{+}\right)\right)^{3 / 2}}-\frac{1}{3 x_{+}^{2} \sqrt{-U^{\prime}\left(x_{+}\right)}}\right)\left(x-x_{+}\right)^{3 / 2} \theta\left(x-x_{+}\right)\right\},
\end{aligned}
$$

где $k_{0}, \Omega(x)$ задаются равенствами (1.16), (1.17). Пусть $z_{-}, z_{+}, z_{-} \sim x_{-}, z_{+} \sim$ $x_{+},-$те точки, в которых $W_{0}\left(z_{ \pm}, h\right)=0$. Тогда

$$
\begin{array}{ll}
z_{-}=x_{-}+h^{1 / 3} x_{-, 1}+O\left(h^{2 / 3}\right), & h \rightarrow 0, \\
z_{+}=x_{+}-h^{1 / 3} x_{+, 1}+O\left(h^{2 / 3}\right), & h \rightarrow 0
\end{array}
$$


где $x_{-, 1}=-k_{0} \Omega\left(x_{-}\right) / U^{\prime}\left(x_{-}\right), x_{+, 1}=k_{0} \Omega\left(x_{+}\right) / U^{\prime}\left(x_{+}\right)$.

Из леммы 7.2 вытекает, что функция $W_{0}$ дважды дифференцируема в точках $x_{-}$, $x_{+}$. Она имеет следующие асимптотики:

$W_{0}(x, h)=W_{0}^{\prime}\left(z_{-}, h\right)\left(x-z_{-}\right)+\frac{W_{0}^{\prime \prime}\left(z_{-}, h\right)\left(x-z_{-}\right)^{2}}{2}+O\left(\left(x-z_{-}\right)^{3}\right), \quad x \rightarrow z_{-}$,

$W_{0}(x, h)=-W_{0}^{\prime}\left(z_{+}, h\right)\left(z_{+}-x\right)+\frac{W_{0}^{\prime \prime}\left(z_{+}, h\right)\left(z_{+}-x\right)^{2}}{2}+O\left(\left(z_{+}-x\right)^{3}\right), \quad x \rightarrow z_{+} ;$

здесь

$$
\begin{aligned}
W_{0}^{\prime}\left(z_{-}, h\right) & =U^{\prime}\left(x_{-}\right)+h^{1 / 3} k_{0} \Phi_{-}+h^{1 / 3} x_{-, 1} U^{\prime \prime}\left(x_{-}\right)+O\left(h^{2 / 3}\right), \\
W_{0}^{\prime \prime}\left(z_{-}, h\right) & =U^{\prime \prime}\left(x_{-}\right)+O\left(h^{1 / 3}\right), \\
W_{0}^{\prime}\left(z_{+}, h\right) & =U^{\prime}\left(x_{+}\right)-h^{1 / 3} k_{0} \Phi_{+}-h^{1 / 3} x_{+, 1} U^{\prime \prime}\left(x_{+}\right)+O\left(h^{2 / 3}\right), \\
W_{0}^{\prime \prime}\left(z_{+}, h\right) & =U^{\prime \prime}\left(x_{+}\right)+O\left(h^{1 / 3}\right)
\end{aligned}
$$

и параметр $h \rightarrow 0$.

Наконец, определим

$$
\begin{aligned}
k_{1} \stackrel{\text { def }}{=} 2\left(1-h^{1 / 3}\left[b_{-}+b_{+}\right]\right)\left(\int_{z_{-}}^{z_{+}} \frac{d x}{\sqrt{W_{0}(x, h)}}\right)^{-1}, \\
W_{1}(x, h) \stackrel{\text { def }}{=} U(x)+h^{1 / 3} k_{1} \int_{z_{-}}^{z_{+}} \frac{1}{\pi\left(x+x^{\prime}\right)} K\left(\frac{2 \sqrt{x x^{\prime}}}{x+x^{\prime}}\right) \frac{d x^{\prime}}{\sqrt{W_{0}\left(x^{\prime}, h\right)}} \\
\quad+\frac{2 h^{2 / 3}}{\pi}\left[\frac{b_{-}}{x+z_{-}} K\left(\frac{2 \sqrt{x z_{-}}}{x+z_{-}}\right)+\frac{b_{+}}{x+z_{+}} K\left(\frac{2 \sqrt{x z_{+}}}{x+z_{+}}\right)\right] .
\end{aligned}
$$

Справедлива

TEOPEMA 7.1. Функиия

$$
\begin{aligned}
S^{\prime}(x, h)= & \sqrt{W_{1}(x, h)}+\frac{1}{\sqrt{W_{1}(x, h)}}\left\{O \left(h ^ { 2 / 3 + 2 / 1 9 } \operatorname { l n } \frac { 1 } { h } \left(1+|\ln | x-z_{-}||\right.\right.\right. \\
& \left.\left.\left.+|\ln | x-z_{+}||\right)\right)+O\left(\frac{h^{8 / 9}}{\left(x-z_{-}\right)^{1 / 3}}\right)+O\left(\frac{h^{8 / 9}}{\left(z_{+}-x\right)^{1 / 3}}\right)\right\}
\end{aligned}
$$

а также константы

$$
k=k_{1}+O\left(h^{1 / 3+2 / 19} \ln \frac{1}{h}\right), \quad \tilde{x}_{ \pm}=z_{ \pm}+O\left(h^{2 / 3} \ln \frac{1}{h}\right)
$$

являются асимптотическим решением задачи (7.2), (7.3), (7.5), (7.9). А именно, заданные формулами (7.17),(7.18) функиия $S^{\prime}(x, h)$ и константы $k, \tilde{x}_{ \pm}$ 
удовлетворяют уравнению (7.9) с точностью $R^{*}$, где $R^{*}$ имеет вид (7.10), условию нормировки (7.5) с точностью $O\left(h^{1 / 3+2 / 19} \ln 1 / h\right)$, а также соотношениям (7.2), (7.3) с точностью $O\left(h^{2 / 3} \ln 1 / h\right)$.

\section{§ 8. Асимптотические собственные значения. Главное приближение}

В этом параграфе мы получим алгебраическое уравнение, из которого находятся асимптотические собственные значения $\lambda_{n}(h)=O(1)$ с точностью $O\left(n^{-7 / 10}\right)$ при $n \rightarrow \infty$ (главное приближение).

Если ограничиться такой точностью, то в правиле квантования (6.9) функцию $S_{0,0}$ можно заменить ее асимптотикой при $\xi \rightarrow+\infty: S_{0,0}(\xi, \tilde{k})=2 \xi^{3 / 2} / 3+$ $O(\sqrt{\xi} \ln \xi)$. Тогда с учетом (7.1) правило (6.9) примет вид

$$
\begin{aligned}
& \frac{1}{h}\left\{\frac{2}{3} \varepsilon^{3 / 2} \sqrt{U^{\prime}\left(\tilde{x}_{-}\right)}+\int_{\tilde{x}_{-+\varepsilon}}^{\tilde{x}_{+-}-\varepsilon} S^{\prime}(x, h) d x+\frac{2}{3} \varepsilon^{3 / 2} \sqrt{-U^{\prime}\left(\tilde{x}_{+}\right)}\right\} \\
& \quad=\pi n+O\left(h^{-1 / 3+13 / 57} \ln \frac{1}{h}\right)
\end{aligned}
$$

где $n$ - целые.

В (8.1) функция $S^{\prime}(x, h)$, а также константы $k, \tilde{x}_{-}, \tilde{x}_{+}$являются решением задачи $(7.2),(7.3),(7.5),(7.9) ; \varepsilon=h^{26 / 57}$.

Далее подставим формулы (7.17),(7.18) в (8.1) и разложим получившееся выражение по степеням $h^{1 / 3}$. Приходим к следующему уравнению для нахождения $\lambda_{n}(h)$ :

$$
\begin{aligned}
& \frac{1}{h} \int_{x_{-}}^{x_{+}} \sqrt{U(x)} d x+\frac{k_{0}}{2 h^{2 / 3}} \int_{x_{-}}^{x_{+}} \frac{\Omega(x)}{\sqrt{U(x)}} d x \\
& \quad+\frac{1}{h^{1 / 3}}\left\{\frac{k_{0}^{3} P_{1}}{8} \int_{x_{-}}^{x_{+}} \frac{\Omega(x)}{\sqrt{U(x)}} d x-\frac{k_{0}^{2} P_{2}}{4}-\frac{k_{0}^{2} P_{3}}{8}+P\right\} \\
&=\pi n+O\left(h^{-1 / 3+1 / 30}\right), \quad h \rightarrow 0 .
\end{aligned}
$$

Здесь $U(x)=\lambda-V(x), \Omega(x)$ имеет вид (1.17), $n$ - целые, а коэффициенты $k_{0}, P_{1}$, $P_{2}, P_{3}, P$ определяются формулами (1.16), (1.18)-(1.21).

Справедлива

ТЕОРема 8.1. Пусть выполнено условие (6.10). Тогда числа $\lambda=\lambda_{n}(h)$, заданные правилом квантования (8.2), являются асимптотическими собственными значениями задачи (1.11)-(1.13) с точностью $O\left(n^{-7 / 10}\right)$ nри $n \rightarrow \infty$. 


\section{Список литературы}

1. Карасев М.В., Перескоков А. В. Асимптотические решения уравнений Хартри, сосредоточенные вблизи маломерных подмногообразий. І. Модель с логарифмической особенностью // Изв. РАН. Сер. матем. 2001. Т. 65. № 5. С. 33-72.

2. Карасев М.В., Маслов В.П. Алгебры с общими перестановочными соотношениями и их приложения. II. Операторные унитарно-нелинейные уравнения // Соврем. пробл. математики. Т. 13. М.: ВИНИТИ, 1979. С. 145-267.

3. Карасев М.В., Перескоков А. В. Логарифмические поправки в правиле квантования. Спектр полярона // ТМФ. 1993. Т. 97. №1. С. 78-93.

4. Карасев M.В., Осипов Ю. В. Собственные функции уравнения Хартри-Фока, не обладающие сферической симметрией // ТМФ. 1982. Т. 52. № 2. С. 263-269.

5. Карасев M. В. Квантовая редукция на орбиты алгебр симметрий и задача Эренфеста. Препринт / ИТФ-87-157Р. Киев: ИТФ АН СССР, 1987.

6. Габдуллин P.P. Маломерная аппроксимация решений уравнения полярона Пекара. Препринт. Пущино: НЦБИ АН СССР, 1991.

7. Бейтмен Г., Эрдейи А. Высшие трансцендентные функции. Т. 3. М.: Наука, 1967.

8. Ильин A. М. Согласование асимптотических разложений решений краевых задач. М.: Наука, 1989.

9. Александров B. M., Мхитарян C. M. Контактные задачи для тел с тонкими покрытиями и прослойками. М.: Наука, 1983.

10. Абрамович, М., Стиган И. (ред.) Справочник по специальным функциям с формулами, графиками и математическими таблицами. М.: Наука, 1979.

Поступило в редакцию

13.III.1998 